\title{
CONTINUOUS AUTHENTICATION BASED ON LEARNING USER COMMAND SEQUENCE
}

\author{
by \\ Bijan Khalilian \\ B.Sc., Ryerson University, Toronto, Ontario, Canada, 2007
}

A Dissertation Submitted in Partial Fulfillment of the Requirements for the

Degree of

MASTER OF SCIENCE

in the Program of Computer Science

Toronto, Ontario, Canada, 2010

(C) Bijan Khalilian 2010

Ryerson University 
I hereby declare that I am the sole author of this thesis or dissertation.

I authorize Ryerson University to lend this thesis or dissertation to other institutions or individuals for the purpose of scholarly research.

I further authorize Ryerson University to reproduce this thesis or dissertation by photocopying or by other means, in total or in part, at the request of other institutions or individuals for the purpose of scholarly research. 


\title{
CONTINUOUS AUTHENTICATION BASED ON LEARNING USER COMMAND SEQUENCE
}

\author{
Bijan Khalilian
}

M.Sc., Computer Science, Ryerson University, 2010

\begin{abstract}
In the context of information and computer security, a masquerader is an individual who can gain access to a system by disguising itself as a legitimate user. One of the prominent and popular methods for authenticating masqueraders is by using an intrusion detection system (IDS). This thesis promotes the idea that learning the user command sequence can be served as an alternative for addressing intrusion detection. Several approaches have been proposed in the literature, where this idea has been explored. To our knowledge, the method by Maxion and Townsend produces the best results of all past techniques so far in terms of detection rate $(82.1 \%$ using the Greenberg dataset). In this thesis, we propose an IDS-based approach that consists in combining a novel Naïve Bayes classifier with a recently proposed sequential sampling technique for continuous authentication, applied to user command sequence, to detect masqueraders. Our experimental evaluation shows that our proposed scheme achieves a detection rate of $98 \%$.
\end{abstract}




\section{Acknowledgments}

It is a pleasure to thank those who made this thesis possible. I owe my deepest gratitude to my supervisor Dr. Isaac Woungang and my co-supervisor Dr. Issa Traore for sharing their knowledge and providing continuous support. Without their help and guidance the completion of this work would have not been possible. I would also like to thank William Zereneh for making his valuable time available to me and many of my colleagues. I am grateful for his timely help and for always keeping his door open for many of us. 


\section{Dedication}

I dedicate this thesis to my loving parents, Hamid and Mansoureh. Without their patience, support, and most of all love, the completion of this work would have not been possible. Furthermore, I would also like to dedicate this work to my wonderful and loving siblings Bita, Anahita and Aria. 


\section{Table of Contents}

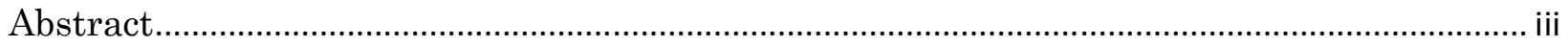

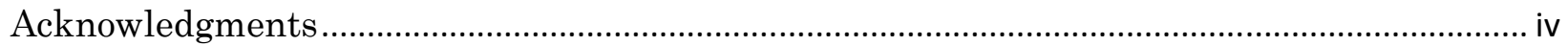

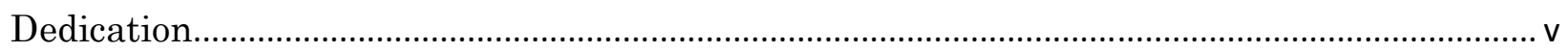

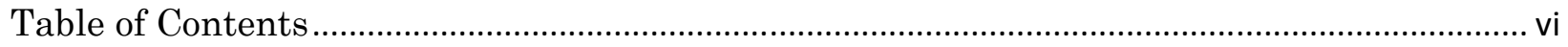

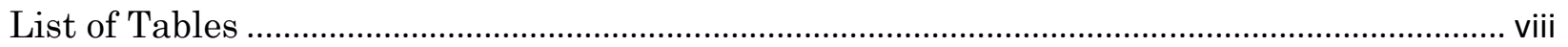

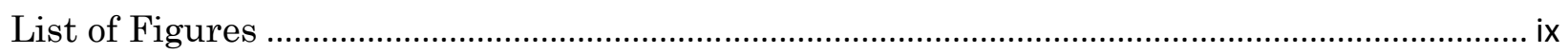

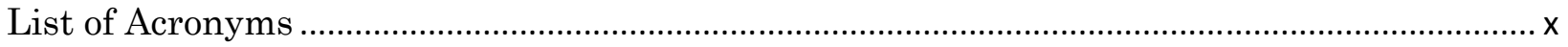

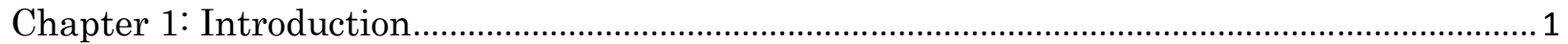

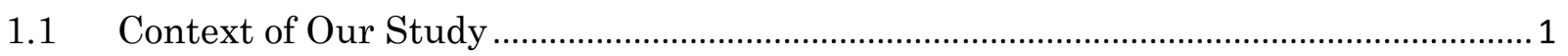

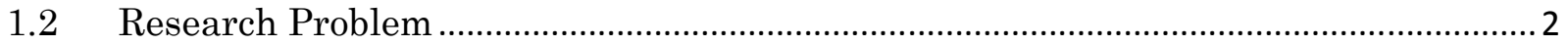

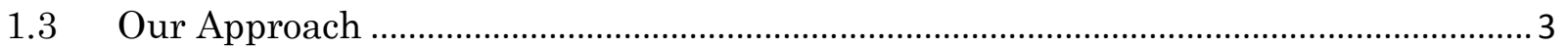

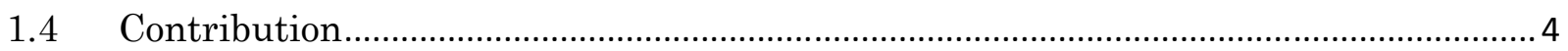

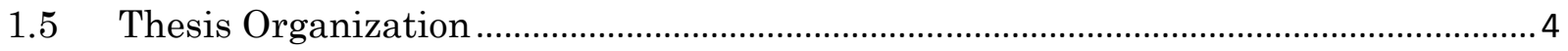

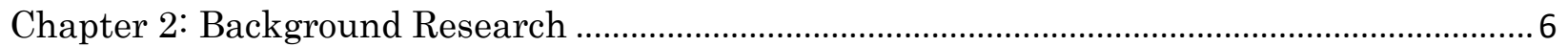

$2.1 \quad$ Intrusion detection System Approaches ............................................................... 6

2.2 Quantitative Modeling Methods................................................................................. 7

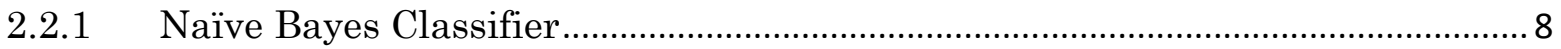

2.2.2 Intrusion Detection Systems in Conjunction with Naïve Bayes Classifier ......... 9

2.3 Continuous Authentication Based on Learning User Command Sequence ..............10

Chapter 3: Continuous Authentication Based on Learning User Command Sequence

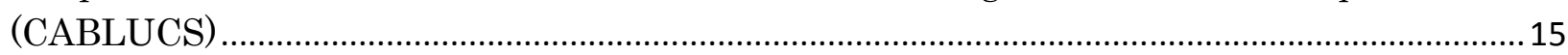

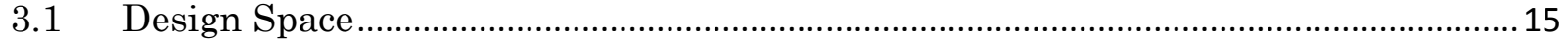

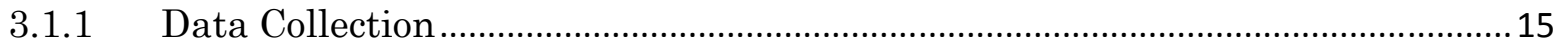

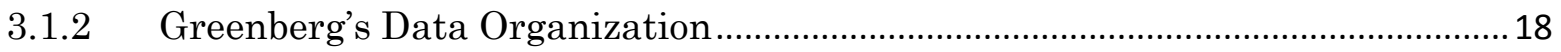

3.1.3 Reproducing Greenberg's Methodology …............................................................. 19

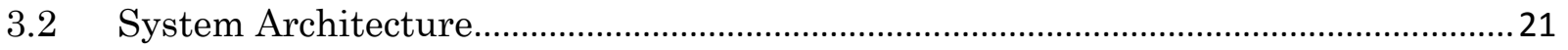

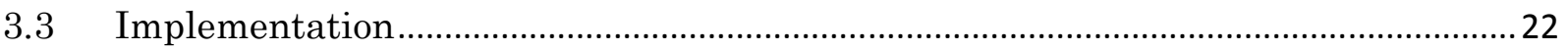

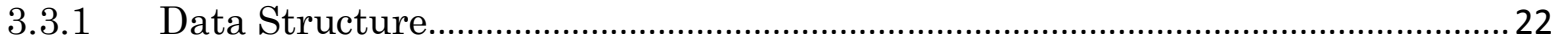

3.3.2 Pre-processing the Dataset............................................................................... 25

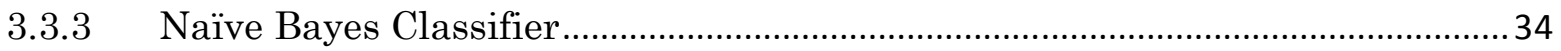




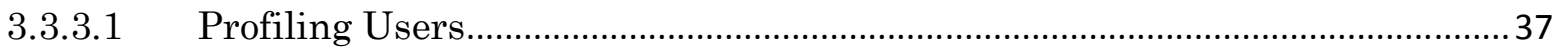

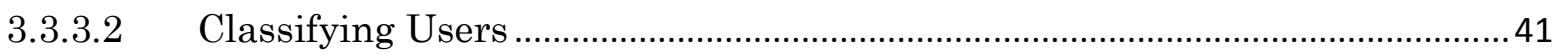

3.3.3.3 Continuous Authentication and the Sequential Sampling Technique ........... 43

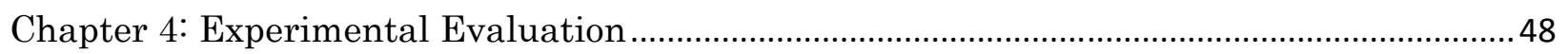

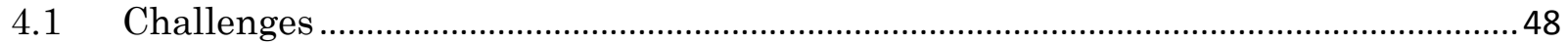

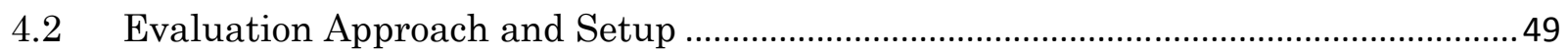

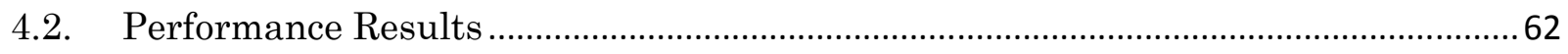

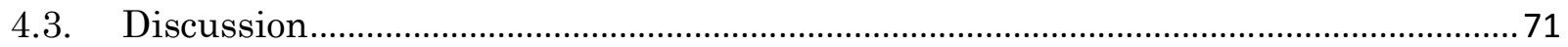

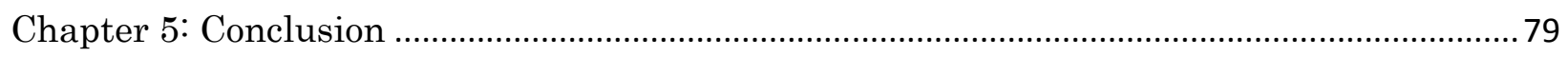

Appendix A: Generated Statistics for the Greenberg Dataset (Ordered by Commands) ........80

Appendix B: Generated Statistics for the Greenberg Dataset (Ordered by History) .............. 83

Appendix C: Generated Statistics for the Greenberg Dataset (Ordered by Errors) ................ 86

Appendix D: Generated Statistics for the Greenberg Dataset (Ordered by Aliases) ...............89

Appendix E: Generated Statistics for the Greenberg Dataset (Ordered by Lines)..................92

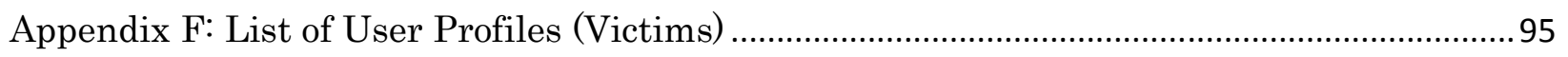

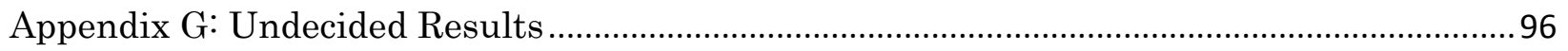

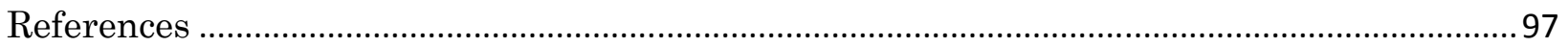




\section{List of Tables}

Table 1: Results produced by 6 methods to detect masquerades. Schonlau et al ....................12

Table 2: Results produced by implementing the Naive Bayes classifier. $[10 ; 26]$..................12

Table 3: A list of detection methods and their relative results ................................................ 13

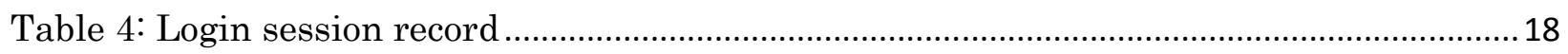

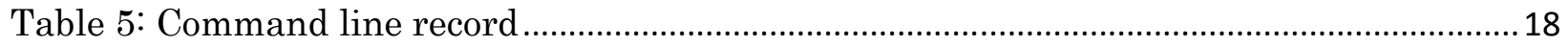

Table 6: Greenberg's reproduced dataset sample................................................................... 20

Table 7: Filename structure used in the dataset........................................................................ 24

Table 8: User types (user_types) database table schema ............................................................26

Table 9: User types (user_types) database table sample data....................................................27

Table 10: Users (users) database table schema .....................................................................2 27

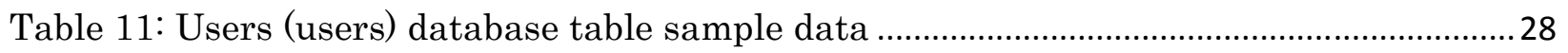

Table 12: Sessions (sessions) database table schema ..................................................................29

Table 13: User sessions (sessions) database table sample data ...................................................30

Table 14: Data items (data) database table schema ......................................................................30

Table 15: Sample command lines in the 'data' table from User 1 .............................................. 32

Table 16: Data report (insert_report) database table schema .................................................... 32

Table 17: Sample reports in the 'insert_report' table from 5 different users ............................34

Table 18: Small segment of a given profile ........................................................................... 41

Table 19: Probability distribution for an arbitrary user ......................................................... 42

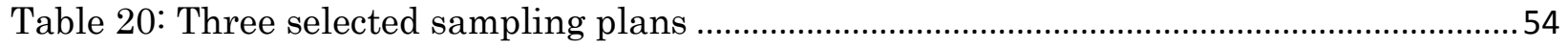

Table 21: engine_report MySQL table schema .......................................................................... 58

Table 22: engine_output MySQL table schema .........................................................................60

Table 23: Details of the final results made by using CABLUCS ...............................................61

Table 24: Successive progression of the decision making process .............................................61

Table 25: List of prepared sampling plans and tests............................................................62

Table 26: Decision making process of the sequential sampling technique...............................65

Table 27: Results achieved based on different parameters and sampling sizes......................67

Table 28: Result comparison based on Cost $=($ FAR $)+($ FRR $)$................................................... 74

Table 29: Result ranking comparison based on Cost $=($ FAR $)+($ FRR $)$....................................... 75

Table 30: Result comparison based on Cost $=(\mathrm{FAR})+6(\mathrm{FRR})$.............................................. 76

Table 31: Result ranking comparison based on Cost $=($ FAR $)+6(\mathrm{FRR})$..................................77

Table 32 :Users that were falsely rejected in 21 different test cases......................................78 


\section{List of Figures}

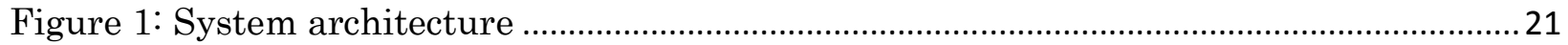

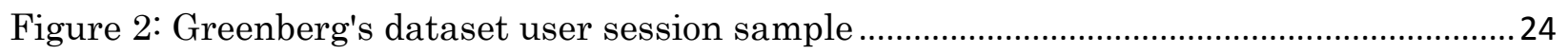

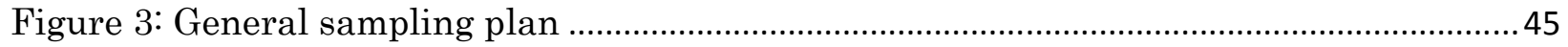

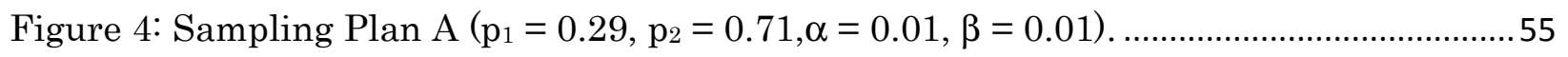

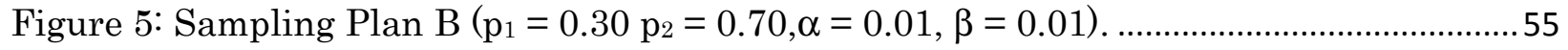

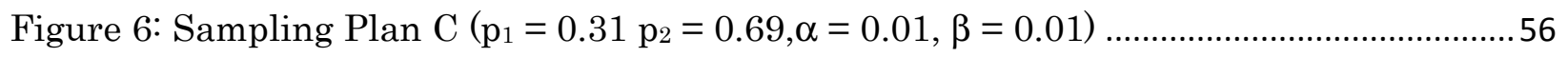

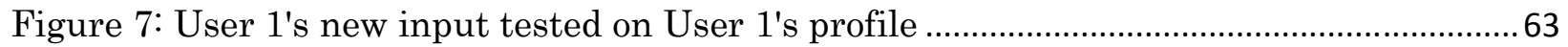

Figure 8: User 2's input tested on User 1's profile ..............................................................64

Figure 9: Analysing the system's FAR rate versus the sample size..........................................68

Figure 10: Analysing the system's FRR rate versus the sample size .......................................69

Figure 11: Analysis of the system's MTTA value as the sample size is increased..................69

Figure 12: Analysing the system's MTTA value versus the sample size .................................70

Figure 13: Analyzing the systems CPU Usage versus the sample size....................................71

Figure 14: Relationship between sample size and FRR/FAR using sampling plan A.............72

Figure 15: Relationship between sample size and FRR/FAR using sampling plan B............72

Figure 16: Relationship between sample size and FRR/FAR using sampling plan C............73 


\section{List of Acronyms}

$\begin{array}{ll}\text { ANN } & \text { Artificial Neural Network } \\ \text { CA } & \text { Continuous Authentication } \\ \text { CR } & \text { Confidence Ratio } \\ \text { DR } & \text { Detection Rate } \\ \text { FAR } & \text { False Accept Rate } \\ \text { FRR } & \text { False Reject Rate } \\ \text { HCS } & \text { Hybrid Command Sequence } \\ \text { IDS } & \text { Intrusion Detection System } \\ \text { CABLUCS } & \text { Continuous Authentication Based on Learning User Command Sequence } \\ \text { MTTA } & \text { Mean Time-to-Alarm } \\ \text { NN } & \text { Neural Network } \\ \text { PHP } & \text { PHP: Hypertext Processor } \\ \text { SVM } & \text { Support Vector Machine } \\ \text { TTA } & \text { Time-to-Alarm }\end{array}$




\section{Chapter 1: Introduction}

\subsection{Context of Our Study}

This thesis introduces a new approach in detecting masquerade attacks in systems, by implementing an intrusion detection system that consists of a Naïve Bayes classifier complemented with a recently proposed sequential sampling technique [1] for continuous authentication, applied to user command sequence. The Naïve Bayes classifier is used to train a dataset that consists of command-line history taken from 168 different users.

\subsection{Motivation}

In general, intrusion detection systems (IDS) are designed to handle masqueraders, i.e. users who impersonate other users, trying to gain access within a secure network. Typically, it is assumed that sophisticated masqueraders possess insider's knowledge on various features of the system such as topologies, potential vulnerabilities and the how various security products have been installed. To protect systems against masqueraders, security technologies such as firewalls, network-based intrusion detection systems, and strong authentication protocols are utilized.

In general, most authentication systems are only concerned about the point of entry [2]. Once a user has successfully passed the initial phase of authentication (i.e. user login), the user is deemed genuine. However, this assumption can be quite costly. In the case where such initial phase of authentication is compromised, the entire system can be in dire jeopardy. IDSs in conjunction with continuous authentication can 
be used to address this problem since these systems have been designed to detect different types of security hazardous behaviours after the user has already been given permission to access the system. The general idea of using continuous authentication is that the legitimacy of an active user session can be validated continuously, leading to a predefined and distinctive user profile (i.e. signature) during a live session within intermediate intervals. To this effect, a continuous authentication system can be used to investigate a user's typing habits [3], mouse dynamics [4] or command line sequence, in order to determine the legitimacy of a given user.

IDSs differ from conventional firewall systems and authentication protocols in the sense that in addition to prevent non-privileged users from accessing sensitive data or performing restricted tasks, they can also be used to control the access capability for users with the appropriate and official privileges who abuse their concessions. In other words, IDSs can be used to detect malicious insiders that use their privileges to perform unauthorized actions. For this reason, IDSs are considered as network security schemes of choice [5;6]. Designing an IDS that can achieve a high level of accuracy while detecting masquerade attacks, is the primary motivation of our work.

\subsection{Research Problem}

Several IDS-based approaches for masquerade detection have been investigated, ranging from approaches based on support vector machine classifiers $[7 ; 8]$; to the

pioneer approaches based on mouse dynamics [1] and keystroke dynamics to approaches based on sequence-based user commands profile $[9 ; 10]$, to name a few. In the latter case, several attempts to learn user command sequence for masquerade detection have been investigated [10]. The proposal that yields the best result so far in 
terms of accuracy (using the Greenberg dataset) - measured by the level of detection achieved, is the work by Maxion and Townsend [10]. Yet, this performance is still inadequate, especially in the context of commercial-based systems. Therefore, designing IDS-based systems that can detect the above-mentioned canonical masquerade attack based on learning sequence-based user commands while producing a better level of accuracy compared to that obtained by the Maxion and Townsend's approach, would be highly desirable. This is the challenge addressed in this thesis.

\subsection{Our Approach}

In this thesis, to address the above-mentioned challenge, we follow up on an idea inherited from the pioneer work in [10], i.e. using a Naïve Bayes classification algorithm to learn sequence-based user commands, with the goal to provide a solution to the problem of masquerade detection. Our approach differs from that presented in [10] with respect to the use of updating mechanisms that dynamically recompute the classifier probabilities as monitored sequences are analyzed and classified by our Naïve Bayes classifier. More precisely, our approach consists of an integration of three components: (1) a data pre-processing module - that captures the user's data input and restructured them to a more manageable format; (2) a detector - which deploys a Naïve Bayes classification algorithm (as in [10]) in order to create a set of distinct user profiles; and (3) a dynamic sampling technique for continuous authentication (so-called sequential sampling technique) - which is inherited from a recently pioneered work on

continuous authentication [1] and is used to distinguish the legitimacy of a given user based on a given user profile. 


\subsection{Contribution}

There have been a lot of works dealing with learning using command sequence to detect masquerade attacks in systems [10]. To our knowledge, the best detection rate achieved so far is attributed to the approach proposed by Maxion and Townsend [10].

The contribution of this thesis is the design of a novel intrusion detection system that learns from sequence-based user commands profile to detect classical masquerade attacks while learning the behavioural tendencies of a given user. This design is realized by complementing a recently proposed evaluation technique for continuous authentication [1] (so-called sequential sampling) with a novel Naïve Bayes learning algorithm, applied to user command sequence. Our approach is shown to achieve a significant improvement over the above-mentioned performance by Maxion and Townsend [10].

\subsection{Thesis Organization}

This thesis is composed of the following Chapters.

\section{Chapter 2: Background Research}

In this chapter, we discuss previous works on the subject and their limitations.

\section{Chapter 3: Continuous Authentication Based on Learning User Command Sequence $\underline{\text { (CABLUCS) }}$}

The chapter constitute the core of this thesis. We describe our CABLUCS intrusion detection system, including a discussion on its implementation. 


\section{Chapter 4: Experimental Evaluation}

Validating the proposed Continuous Authentication Based on Learning User Command Sequence (CABLUCS) scheme is of course an essential part of this research work. In this chapter, we describe the experimental setup as well as the performance parameters and the obtained results.

\section{Chapter 5: Conclusion}

We conclude our work and present future possible works that can be done to extend the scope of the content of this thesis. 


\section{Chapter 2: Background Research}

This chapter discusses related works on intrusion detection systems. Common methods and models employed within this field of research are discussed, as well as related research challenges. Finally, our new approach is contrasted against these related works.

\subsection{Intrusion detection System Approaches}

Various design approaches to Intrusion Detection Systems (IDSs) have been proposed in the literature, as well as a few attempts to produce taxonomies of IDSs [11], [12; 13; $14 ; 15 ; 16 ; 17]$, Typically, IDSs can be classified into three categories: sensors, detectors, and positive intrusion handlers (not including false alarms) [11]. Researches focus their attention mostly on detector entity along the system characteristics. There are currently two major types of detection approaches: Anomaly Detection and Signature Detection (Misuse Detection).

Anomaly Detection is based on abnormal behavior. It relies on self-learning for the purpose of detecting abnormal behaviors. A drawback of such system is that some behaviors may not be undesirable, leading to a high false positive rate [11]. Selflearning systems are broken down into two categories: non-time series systems and time series ones. Non-time series systems use stochastic models to determine what a normal behavior would be disregarding time constraints. On the other hand, timeseries systems determine normality based on techniques such as Markov models, artificial neural networks, to name a few $[11 ; 18]$. As instance, sequence learning for anomaly detection is an example of approach that records the normal working state of a 
system (i.e. the system's call traces, network packet traces, resource consumption patterns) with regards to its user, then uses this dataset to differentiate between normal and suspicious behavior, by comparing expected behavior patterns with lively detected behavior patterns. The goal is to produce a cost-effective and preeminent model that can, swiftly and appropriately be managed by a certain mechanism. This latter criteria is important because once these systems are deployed in large organizations, the datasets can be very large and in some cases unreliable in terms of timely masquerade detection.

Misuse detection systems are based on previously known intrusion attempts that are fairly common; therefore they may not be reliable in terms of catching new malicious behaviors. However, these systems can be used to detect sequences of instructions that violate the security policies. They make use of rule sets to distinguish behaviors; thus are unable to detect violations that are unknown to these rule sets.

\subsection{Quantitative Modeling Methods}

For quantitative modeling purpose, artificial neural networks are considered as an important class of tools [19]. These types of systems or computing models have been applied to various problems in many different areas, particularly for identifying the fundamental relationships among a set of variables or patterns in the data [19]. Two important characteristics of these systems are: parallel processing of information and learning and generalizing from experience. 


\subsubsection{Naïve Bayes Classifier}

In addition to artificial neural network, Bayesian learning algorithms have been used as a tool of choice for modeling various systems [19]. The Bayesian learning concept consists in inferring a set of parameters of a predefined model from the information contained in some data.

The Naive Bayes classifiers are among the most successful known class of Bayesian learning algorithms, for learning to classify text documents [20; 21]. The Naïve Bayes classifier is also widely used to detect and classify spam [23; 24] and many other unwanted electronic documents.

The Naïve Bayes classifier is directly related to tasks where each instance $x$ is described by a combination of attribute values. The target function $f(x)$ can represent any available value from a finite set $V$. Given a set of training examples of the target function, the algorithm can classify a new instance. More precisely, given the new instance tuple of attribute values $\left(a_{1}, a_{2} \ldots a_{n}\right)$, the classifier indicates the most probable target value $V_{M A P}$ as follows:

$$
V_{M A P}=\operatorname{argmax}_{v_{j} \in V} P\left(v_{j} \mid a_{1}, a_{2} \ldots a_{n}\right)
$$

By applying the Bayes theorem, Equation 1 can be re-formulated as: 


$$
\begin{gathered}
V_{M A P}=\underset{v_{j} \in V}{\operatorname{argmax}} \frac{P\left(a_{1}, a_{2} \ldots a_{n} \mid v_{j}\right) P\left(v_{j}\right)}{P\left(a_{1}, a_{2} \ldots a_{n}\right)} \\
=\operatorname{argmax}_{v_{j} \in V} P\left(a_{1}, a_{2} \ldots a_{n} \mid v_{j}\right) P\left(v_{j}\right)
\end{gathered}
$$

Given the training data, an estimation can be made using the two terms $P\left(a_{1}, a_{2} \ldots a_{n} \mid v_{j}\right)$ and $P\left(v_{j}\right)$. In order to evaluate $P\left(v_{j}\right)$, we calculate the frequency of which the target value $v_{j}$ appears in the training examples.

The Naïve Bayes classifier is built on the assumption that the tuple of attribute values $\left(a_{1}, a_{2} \ldots a_{n}\right)$ is conditionally independent given the target value [20]. Therefore, this naive assumption indicates that $P\left(a_{1}, a_{2} \ldots a_{n} \mid v_{j}\right)=\prod_{i} P\left(a_{i} \mid v_{j}\right)$ and hence the Naïve Bayes classifier is expressed as:

$$
V_{N B}=\operatorname{argmax}_{v_{j} \in V} P\left(v_{j}\right) \prod_{i \in \text { positions }} P\left(a_{i} \mid v_{j}\right)
$$

The Naïve Bayes classifier has a specific characteristic that is different from other learning algorithms. The hypothesis is evaluated by examining the frequency of different data combinations throughout the training examples and without the need of querying. A comprehensive description of this machine learning algorithm can be found in [20]. The procedural steps required to implement the Naïve Bayes classifier is detailed in Chapter 3.

\subsubsection{Intrusion Detection Systems in Conjunction with Naïve Bayes Classifier}

Typically, the Naïve Bayes classifier is used to classify text documents [25]. However, a successful implementation of a Naïve Bayes classifier in an intrusion detection environment has also been presented $[26 ; 27 ; 6 ; 10 ; 28]$. Due to the nature of this 
learning algorithm, it is natural to employ it in an application where the learning involves strings of text, such as command-lines. A description of procedures involved to implement such machine learning algorithm in an IDS based on learning user command-line sequences is given in Chapter 3.

\subsection{Continuous Authentication Based on Learning User Command Sequence}

The source of input data used in anomaly detection is normally extracted from different types of user/system input. The most commonly used data source in anomaly based intrusion detection systems involves one of, mouse dynamics [4; 29], keystroke dynamics, system processes [30;31;32;33;34], and/or command line sequence. In most literatures that involve learning command line sequence, the data is produced using UNIX or UNIX-like operating systems. The most commonly used dataset in this field, is the work of Schonlau et al. [9]. In the work of Schonlau et al., six different methods were used in order to learn and profile [35] user behaviour, based on their given UNIX command line history. These methods include:

- Uniqueness

- Bayes one-step Markov

- Hybrid multi-step Markov

- Compression

- IPAM (Incremental Probabilistic Action Modeling)

- Sequence-match 
The uniqueness method is established based on the command frequency in the training data. A command line that is not witnessed in the training data is deemed to be malicious. Commands that have a low frequency in the training data will demonstrate a higher indication of malicious behaviour.

The Bayes one-step Markov method is based on the concept of single iterations between commands. The system will compare the given sequence of iteration probabilities to previously known iteration tendencies and determine the legitimacy of the given user.

The hybrid multi-step Markov method is based on the $\mathrm{n}^{\text {th-order }}$ Markov chain and a given model that determines the proportionality of commands that were not witnessed in the training data.

The compression method is based on generating reversible maps for the data in correspondence to a representation that utilizes less storage than the original. New input from the user is compressed and compared to the given maps and tested for legitimacy based on the compression rates.

The IPAM (Incremental Probabilistic Action Modeling) method is based on one-step command iteration probabilities with regards to a given training data, while continuously expanding and updating its arrangement.

The sequence-matching method is based on determining the similarities between the ten most recent commands of a given user in comparison with a user's profile. The following table demonstrates the results achieved in each implemented method by Schonlau et al. [9]. 
Table 1: Results produced by 6 methods to detect masquerades. Schonlau et al

\begin{tabular}{|l|c|c|c|}
\hline Method & FRR (\%) & FAR (\%) & DR (\%) \\
\hline Uniqueness & 1.4 & 60.6 & 39.4 \\
\hline Bayes one-step Markov & 6.7 & 30.7 & 69.3 \\
\hline Hybrid multistep Markov & 3.2 & 50.7 & 49.3 \\
\hline Compression & 5.0 & 65.8 & 34.2 \\
\hline Sequence-Match & 3.7 & 63.2 & 36.8 \\
\hline IPAM & 2.7 & 58.9 & 41.1 \\
\hline
\end{tabular}

Looking at the results produced by Schonlau et al., we can clearly observe that the Uniqueness method has the lowest False Rejection Rate (FRR), while lacking a convincing False Acceptance Rate (FAR). Although the FRR value is relatively low, the chance of detecting malicious behaviour (DR) is $39.4 \%$.

Other recent work done in this field includes the work of Maxion and Townsend [10]. Using the Naïve Bayes classifier as their learning algorithm, they have produced encouraging results. The following table demonstrates their final results after testing their method against both the Schonlau et al. dataset (typically denoted as SEA) and the Greenberg [36] dataset.

Table 2: Results produced by implementing the Naive Bayes classifier. [10; 26]

\begin{tabular}{|l|c|c|c|l|}
\hline Method & FRR (\%) & FAR (\%) & DR (\%) & Dataset \\
\hline Naïve Bayes (updating) & 1.3 & 38.5 & 61.5 & SEA \\
\hline Naïve Bayes (no-updating) & 4.6 & 33.8 & 66.2 & SEA \\
\hline Naïve Bayes (truncated) & 4.7 & 29.1 & 70.9 & Greenberg \\
\hline Naïve Bayes (enriched) & 5.7 & 17.9 & 82.1 & Greenberg \\
\hline
\end{tabular}

The hybrid command sequence (HCS) [37] model is another method used in order to detect malicious behaviour based on learning user command sequence. By using a genetic algorithm, the model profiles users based on recorded sessions. It evaluates users considering multiple command sequence fragments in a single session [37]. 
Other detection methods with regards to learning command sequences include the use of SVM (Support Vector Machine) [7; 38]. SVM is a pattern recognition classifier. It has shown significant results in terms of producing high detection rates $[7 ; 38]$. However the FRR rates are still considered to be high.

The following table demonstrates a comprehensive statistical look at the results gained from implementing each of the mentioned methods with respect to a given dataset.

Table 3: A list of detection methods and their relative results

\begin{tabular}{|l|c|c|c|l|}
\hline Method & FRR (\%) & FAR (\%) & DR (\%) & Dataset \\
\hline Naïve Bayes (updating) [10] & 1.3 & 38.5 & 61.5 & SEA \\
\hline Naïve Bayes (no-updating) [10] & 4.6 & 33.8 & 66.2 & SEA \\
\hline Uniqueness [9] & 1.4 & 60.6 & 39.4 & SEA \\
\hline Bayes one-step Markov [9] & 6.7 & 30.7 & 69.3 & SEA \\
\hline Hybrid multistep Markov [9] & 3.2 & 50.7 & 49.3 & SEA \\
\hline Compression [9] & 5.0 & 65.8 & 34.2 & SEA \\
\hline Sequence-Match [9] & 3.7 & 63.2 & 36.8 & SEA \\
\hline IPAM [9] & 2.7 & 58.9 & 41.1 & SEA \\
\hline SVM (RBF Kernel) [7] & 9.7 & 19.9 & 80.1 & SEA \\
\hline SVM (K-gram Kernel) [38] & 14.19 & 10.39 & 89.61 & SEA \\
\hline SVM (String Kernel) [38] & 23.77 & 2.6 & 97.40 & SEA \\
\hline HCS [37] & 33.9 & 1.4 & 98.6 & SEA \\
\hline Naïve Bayes (truncated) [26] & 4.7 & 29.1 & 70.9 & Greenberg \\
\hline Naïve Bayes (enriched) [26] & 5.7 & 17.9 & 82.1 & Greenberg \\
\hline
\end{tabular}

These methods can be evaluated and ranked based on certain ranking functions [39; 9; 6; 26]. These ranking functions depend solely on certain predefined criteria. Depending on the application, the significance of the errors produced by each detection method can vary. The ranking functions involved in determining the quality of a detection method is discussed in more detail in further chapters. 
The detection rates (DR) in most of the mentioned methods are very low. In cases where the detection rate is above $90 \%$ the FRR rates are above $20-30 \%$. The challenge is to develop a system that would notably reduce the FAR and FRR rates.

In this thesis, we propose a novel IDS termed as Continuous Authentication Based on Learning User Command Sequence (CABLUCS). Our approach consists of using the sequential sampling technique (a novel proposed evaluation technique for continuous authentication [1]) in conjunction with the Naïve Bayes learning, applied to user command sequence, to detect masquerade attacks while learning the behavioural tendencies of a given user. More precisely, the user's normal behaviours are recorded and profiled using the Naïve Bayes classifier. The generated profile is used as their signature, while individuals whose behavioural tendency fails to match the given signature are identified as masqueraders. 


\section{Chapter 3: Continuous Authentication Based on Learning User Command Sequence (CABLUCS)}

This Chapter constitutes the main contribution of this thesis. Here, we describe the design of our proposed Continuous Authentication Based on Learning User Command Sequence (CABLUCS) scheme. The design space, system architecture, and data collection and processing methodologies are described in-depth. A typical intrusion scenario is also introduced to assess the stated design.

\subsection{Design Space}

Designing an IDS involves a few challenges, including the methods involved in implementing data collectors, detectors and the different responses offered by the intrusion handlers.

\subsubsection{Data Collection}

Data collection is an important part of the system. Sensors are placed in appropriate locations within the system, in order to listen to the system's activities and collect important data that will determine whether or not an intrusion has taken place. However, depending on the native system, this task can be rather difficult. Learning user command sequences will be a challenge in terms of being able to analyze this data in such a way as to produce the appropriate analysis of the active situation, which will then serve to take the proper actions. In UNIX based systems or other similar systems, one can take advantage of the input provided by the user within a shell (a separate software program that provides direct communication between the user and the operating system). This data is collected by the operating system and is usually defined 
by the term shell history. Although datasets can be controversial in terms of privacy issues [40], in most cases, shell history is readily available.

The shell history can be used to achieve a basic understanding of the user's common patterns. Typically, the command-line history is used when attempting to develop an IDS within a UNIX environment $[26 ; 10 ; 41 ; 42]$. In 1988 , Dr. Saul Greenberg of the Department of Computer Science at the University of Calgary, has collected traces of 168 users using the UNIX $\mathrm{C}$ shell $(c s h)$. These traces correspond to command line data executed by each user and the data was intended to be used for research purposes. This dataset [36] was kindly granted to us and we have used it in this thesis. Running this dataset using a slight modification of the $\mathrm{C}$ shell (csh) command interpreter has enabled us to duplicate Dr. Greenberg's data collection method, which is crucial in the context of this thesis in order to accomplish our data collection objectives.

Data collection in correspondence to different categories and groups of subjects is of importance. In order to relate different behaviours, it is important to have certain understanding of the given subjects, in which the data is being collected from. In this case, subjects were 168 unpaid volunteers, either students or employees of the University of Calgary. Subjects are divided by Greenberg into 4 different groups, which include:

- Novice Programmers

- This group consisted of individuals that had no prior programming experience, minimal knowledge of operating systems or UNIX-like command interpreters. These users spent the majority of their time 
learning programming techniques and concepts, while familiarizing themselves with the system's facilities [36].

- Experienced Programmers

- This group consisted of undergraduate Computer Science students completing their senior years. An understanding of the UNIX environment and moderate knowledge of programming languages were expected from this group. [36].

- Computer Scientists

- This group consisted of Computer Science graduates, including the members of the Faculty, researchers and past graduates from the Department of Computer Science [36].

\section{- Non-programmers}

- This group consisted of members that mostly concentrated on the use of word processing applications. These members had little or no experience in programming languages or no knowledge of the UNIX environment [36].

It should be acknowledged that subjects were assigned as members of these groups given their current agenda at the University of Calgary. Therefore, the assumption that all members fit the given criteria of a particular group cannot be made thoroughly. From the months of February 1987 through June 1987, command line data was continuously collected on site, at Dr. Greenberg's laboratory. The collected data had a specific formatting that includes different annotation for explaining certain situations 
that had incurred during data collection. These annotations along certain drawbacks to the collected data are discussed next.

\subsubsection{Greenberg's Data Organization}

The given data was organized through hierarchal folders. The base folder was named as unix_data. This base folder was composed of five subfolders. Four of these subfolders corresponded to the groups of subjects, which themselves stored all command trace data of every subject (e.g. novice programmers, experienced programmers, computer scientists, and non-programmers).

The fifth subfolder, showerrorcode, included a C program, which was designed to provide explanations for the error codes that were generated by users when executing certain command-lines.

The following tables give a brief description of the attributes used in this dataset.

Table 4: Login session record

\begin{tabular}{|l|ll|}
\hline Code & Description & Example \\
\hline $\mathbf{S}$ & Start time of the login session & S Thu Sep 20 14:23:32 2008 \\
\hline E & End time of the login session & E Thu Sep 20 19:11:12 2008 \\
\hline
\end{tabular}

Table 5: Command line record

\begin{tabular}{|lll|}
\hline Code & Description & Example \\
\hline C & The line entered by the user & C gedit document.txt\& \\
\hline D & The current working directory & D /home/user/documents/ \\
\hline
\end{tabular}




\begin{tabular}{|lll|}
\hline A & $\begin{array}{l}\text { The alias expansion of the previous command } \\
\text { (if any) }\end{array}$ & A NIL \\
\hline $\mathbf{H}$ & $\begin{array}{l}\text { The line entered had a history expansion in it } \\
\text { (True or Nil) }\end{array}$ & H NIL \\
\hline $\mathbf{X}$ & $\begin{array}{l}\text { The error detected in the line by csh (if any). A } \\
\text { following letter and number code indicates the } \\
\text { category and actual error type. }\end{array}$ & X NIL \\
\hline $\mathbf{T}$ & $\begin{array}{l}\text { The time the command line was executed by } \\
\text { the command interpreter. }\end{array}$ & T Thu Sep 20 16:11:43 2008 \\
\hline
\end{tabular}

\subsubsection{Reproducing Greenberg's Methodology}

In order to understand the data collection mechanism used by Greenberg et al. [36], we had to reproduce it using the software package that was kindly granted to us. C shell is a UNIX command interpreter that introduced new features such as aliases and command history. This justifies (in some sense) why Dr. Greenberg used this shell in order to collect command line history.

In order to achieve a consistent duplication of work, we also use $\mathrm{C}$ shell to reproduce Dr. Greenberg's data collection mechanism. Although this tool may be considered inadequate compared to more recent command interpreters, it is important to note that it serves its purpose in the case of collecting command line history. In order to reproduce Dr. Greenberg's data collection mechanism, a copy of the C shell source code was acquired from one of the Ubuntu's available repositories within our laboratory ${ }^{1}$.

\footnotetext{
${ }^{1}$ The Distributed Applications and Broadband Network laboratory (DABNEL), Department of Computer Science, Ryerson University, Toronto, Canada
} 
After making appropriate modifications to the $\mathrm{C}$ shell code provided by Greenberg et al. [36], sensors were placed accordingly in order to produce similar results. In addition to Dr. Greenberg's selection of attributes, a new attribute called Time (denoted T) is introduced. This attribute is used to determine the system time that the command line was executed by the command interpreter. Although Dr. Greenberg had included the attributes $S$ and $E$ which denote the starting and the ending time of each session respectively, it appeared important for us to track the displacement time $\Delta T$ of each command line. This is done in order to gain a better understanding of the user's intentions.

The output of our modified csh scheme compared to that of the Greenberg dataset scheme is captured in Table 6.

Table 6: Greenberg's reproduced dataset sample
Greenberg Sample Data
Reproduced Sample Data
C $l_{S}$
D /home/XXXX/documents/
A $l_{s}-l a$
$\mathrm{H} N I L$
$\mathrm{X} N I L$
C $1 s$
D /home/XXXX/documents/
A $l_{S}-l a$
$\mathrm{H} N I L$
$\mathrm{X} N I L$
T Thu Sep 20 16:11:432008

Few drawbacks of the Greenberg's approach for data collection [36] are as follows.

- Given the structure of the implementation, the "details of history directives were not recorded" [36]. However, there are indications of history being used and the command-line that was retrieved. 
- It is important to acknowledge that the system was unable to capture all user activity. This mainly relates to software packages that are invoked by the user, where the command line is no longer used (e.g. emacs versus $l_{S}$ ).

- The command line executed does not necessarily determine the program that was actually invoked. Because of the many ways a program can be invoked (e.g. through an alias or a script). Although the records for the alias used are included in the dataset, the dataset fails to compensate for events where an alias is used to invoke another alias.

\subsection{System Architecture}

Similarly to many existing intrusion detection systems, our architecture is composed of sensors, detectors and intrusion handlers as depicted in Figure 1.

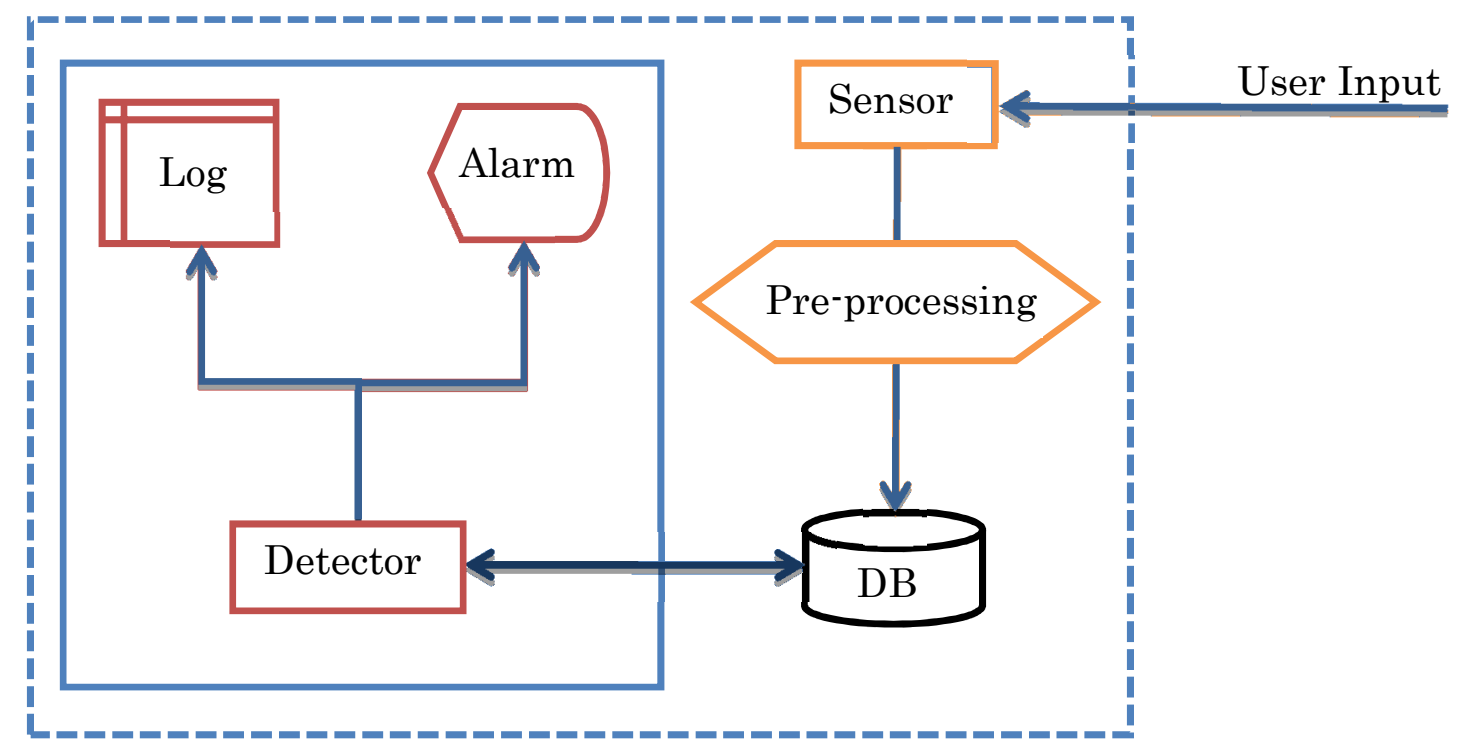

Figure 1: System architecture 
The user input is captured by a sensor and restructured into a desired format (by undergoing a pre-processing step). The output of the pre-processing step is then placed in an incoming pool in the database and made ready for use. The detection mechanism (so-called Detector) will then deploy its intrinsic evaluation algorithm (in the form of a Naïve Bayes classifier) and a decision of an acceptance or a rejection will be made. If the input is rejected, the system will be alarmed and appropriate actions will be taken. Meantime, the system will keep maintaining a Log file that stores all the activities that have been running the system's operations. In case of an acceptance, the users will continue to use their concessions while the system will continue to authenticate their behaviours.

\subsection{Implementation}

This section describes the implementation of the Continuous Authentication Based on Learning User Command Sequence (CABLUCS) design approach. The Greenberg dataset [36] is used as the source for generating user profiles and user inputs. The Naïve Bayes classifier is used in conjunction with a new evaluation technique based on continuous authentication [1], namely the sequential sampling technique, to classify and evaluate a given user.

\subsubsection{Data Structure}

The Greenberg dataset is used as the source of data for this implementation, for training and testing purposes.

The data is in its raw format. It consists of multiple folders, each representing a separate category of subjects. The categories are defined by each user's level of 
experience or position (i.e. novice programmers, experienced programmers, computer scientists, and non-programmers) within the set of test subjects. For the purpose of our implementation, the category under which the user falls into is ignored. This information may be useful for the implementations of certain IDSs. However, due to the nature of our approach, the user categories are deemed to be extraneous. A discussion on future works that can incorporate a primary and secondary levels of classification in a multi-category based user environment is given in the Conclusion Chapter.

Each user is separated with a text file that contains the recorded command line history of the user. Each session is separated by a start and end time stamp. Figure 2 illustrates a single user session. 
S Wed Feb 18 16:37:25 1987

E Wed Feb 18 16:56:22 1987

C date

D /user/srdg/xxxxx

A NIL

H NIL

X NIL

C mail

D /user/srdg/xxxxx

A NIL

H NIL

X NIL

C p audio.mail

D /user/srdg/xxxxx

A page audio.mail

H NIL

Figure 2: Greenberg's dataset user session sample

The filename for each user is constructed using the name of the category that the user is part of, and is concatenated with a numerical digit. An example is given in Table 7 .

Table 7: Filename structure used in the dataset

User Category

Computer Scientist
Filenames

scientist -1 , scientist- $2, \ldots$, scientist- 52 
Experienced Programmers

Non-Programmers

Novice Programmers experienced-1, experienced-2, .., experienced-36

non- 1 , non- $2, \ldots$, non-25

novice- 1 , novice- $2, \ldots$, novice- 55

In this dataset (Table 7), there are 168 users, among which 52 are Computer Scientists, 36 are Experienced Programmers, 25 are Non-Programmers and 55 are Novice Programmers.

\subsubsection{Pre-processing the Dataset}

Due to the difficulty encountered in using the data in its current raw format, we had to restructure the data into a more manageable configuration. To this effect, the data was pre-processed and restructured into a relational database. This step is vital in order to maintain the relations between the command-lines, sessions, users and the user categories while this process is being completed. During the pre-processing progression, the complete structure and integrity of the data was preserved and tested.

The dataset was restructured into four database tables, referred to as user_types, users, sessions and data (as shown in Tables 8 to 14).

- The user_types table (Table 8) is created in order to maintain the different user categories involved in the dataset. This table can also be used to merge other datasets of similar nature into Greenberg's dataset by introducing new sets of categories. 
Table 8: User types (user_types) database table schema

\begin{tabular}{|l|l|}
\hline Field Name & Description \\
\hline utid & This field maintains the id for each user \\
& category. This id is used in other tables \\
& to indicate which category a particular \\
& user belongs to. \\
\hline type & This field defines a two character \\
& identification of a user type. (i.e. 'cs' for \\
& Computer Scientist) \\
\hline description & This field is used to maintain a brief \\
& description of each user type. It is used \\
& for the purpose of describing the types \\
& in English for individuals who are new \\
& to using the Greenberg dataset. \\
\hline
\end{tabular}

- The user_types table (Table 9) maintains the four records that directly correspond to the four different categories involved in the dataset. 
Table 9: User types (user_types) database table sample data

\begin{tabular}{|lll|}
\hline utid & type & description \\
\hline 1 & cs & Computer Scientist \\
\hline 2 & $\mathrm{ep}$ & $\begin{array}{l}\text { Experienced } \\
\text { Programmer }\end{array}$ \\
\hline 3 & $\mathrm{np}$ & Non-Programmer \\
\hline 4 & $\mathrm{nv}$ & Novice Programmer \\
\hline
\end{tabular}

These values in Table 9 are static. They are used as a reference point to indicate which category a user belongs to.

- In order to maintain the identity of each user, the users' table (Table 10) is introduced. This table holds the basic records of all 168 users involved in the dataset. The uid field is used consistently throughout the database in order to maintain the data integrity.

Table 10: Users (users) database table schema

\begin{tabular}{|l|l|}
\hline Field Name & Description \\
& This field maintains the id for each \\
& user. This id is used in other tables to \\
& identify each user. \\
\hline utid & This field is used to indicate which \\
& category a particular user belongs to \\
& within the user_type table. \\
\hline greenberg_name & This field maintains the filename used \\
& in the Greenberg dataset that \\
\hline
\end{tabular}


corresponds to the given user. (i.e. scientist-1)

- The users table (Table 11) can also be used as a quick reference in order to distinguish between the different users while manually traversing through the database. This table plays a major role in keeping the integrity of the restructured dataset. Manipulation of this table can compromise the integrity of the overall dataset and hence it is only used as a reference.

\section{Table 11: Users (users) database table sample data}

\begin{tabular}{|lll|}
\hline Uid & Utid & greenberg_name \\
\hline 1 & 1 & scientist -1 \\
\hline 2 & 1 & scientist- 2 \\
\hline 3 & 2 & experienced -1 \\
\hline 4 & 3 & programmer-1 \\
\hline 5 & 4 & non-1 \\
\hline
\end{tabular}

- Sessions were handled with two lines at the beginning of each of the sessions. Each session's start and end time was parsed and converted into a UNIX Timestamp, and then inserted into the sessions table (Table 12). It is important to notice that once the newly converted timestamp is made available, date, time calculations and manipulations become simpler. 
Table 12: Sessions (sessions) database table schema

\begin{tabular}{|l|l|}
\hline Field Name & Description \\
& This field maintains the session id \\
& for each session within a user's \\
& stored data. This id is used in other \\
& tables to identify a session. \\
\hline uid & This field is used to indicate the \\
& user that this session belongs to. \\
\hline start & This field indicates the start time of \\
& a session. The values are kept in \\
& UNIX Timestamp format. \\
\hline end & This field indicates the start time of \\
& a session. The values are kept in \\
& UNIX Timestamp format. \\
\hline
\end{tabular}

A UNIX Timestamp is an integer which indicates the number of seconds elapsed since midnight proleptic Coordinated Universal Time (UTC) of January 1, 1970 [43]. For example, the UNIX Timestamp of 540682645 is the equivalent of February 18, 1987 at 3:37:25 pm. Every data item (command line) belongs to a particular session. Using the sessions table, we can immediately identify such session's start and end time/date as shown in Table 13. 
Table 13: User sessions (sessions) database table sample data

\begin{tabular}{|c|c|c|c|}
\hline sid & Uid & start & end \\
\hline 1 & 1 & 540682645 & 540683782 \\
\hline 2 & 1 & 540742586 & 540744368 \\
\hline 3 & 2 & 544203016 & 544203669 \\
\hline 4 & 2 & 544424264 & 544438849 \\
\hline 5 & 2 & 544449748 & 544459510 \\
\hline
\end{tabular}

- The data table (along with its relations with the user_types, users and sessions tables) holds the entire dataset. It contains 303,628 data items (command-lines) from 168 different users. Its main fields are shown in Table 14.

Table 14: Data items (data) database table schema

\begin{tabular}{|l|l|}
\hline Field Name & Description \\
& This field maintains the data id for \\
& each command line in the \\
& Greenberg dataset. This id is used \\
& in other tables to identify a \\
& command line. \\
\hline sid & This field indicates which session \\
& this command line belongs to. \\
& Using this id we can indicate the \\
& start and end time of the session. \\
\hline
\end{tabular}




\begin{tabular}{|l|l|}
\hline uid & This field is used to indicate the \\
& user that this command line \\
& belongs to. \\
\hline order & This field is used to maintain the \\
& order in which command lines in a \\
& session were entered. \\
\hline command & This field contains the entire \\
& command line. \\
\hline directory & This field contains the current \\
& working directory in which the \\
& command line was executed. \\
\hline This field will indicate if the \\
command line was in fact an alias \\
to execute another program. It \\
will contain the command in which \\
the alias is executing otherwise a \\
this value will be given. \\
\hline This field indicates whether or not \\
occurred during the executing of \\
command line. \\
\hline error
\end{tabular}


- The data table (Table 15) encompasses the completely restructured dataset. In its new format, the data can be searched, manipulated and tested at a higher rate of efficiency. Furthermore, this higher rate of efficiency can also be transferred onto any available platforms.

Table 15: Sample command lines in the 'data' table from User 1

\begin{tabular}{|l|l|l|l|l|l|l|l|l|}
\hline did & sid & uid & order & command & directory & alias & history & error \\
\hline 1 & 1 & 1 & 1 & Date & /user/srdg/xxxxx & NIL & NIL & NIL \\
\hline 2 & 1 & 1 & 2 & Mail & /user/srdg/xxxxx & NIL & NIL & NIL \\
\hline 3 & 1 & 1 & 3 & p audio.mail & $/$ user/srdg/xxxxx & $\begin{array}{l}\text { page } \\
\text { audio.mail }\end{array}$ & NIL & NIL \\
\hline 4 & 2 & 1 & 1 & Ls & /user/srdg/xxxxx & /bin/ls -Fs & NIL & NIL \\
\hline
\end{tabular}

In order to gain a better understanding of the simple statistical features of the dataset, the insert_report table (Table 16) is introduced. This table gives a general understanding on the number of command-lines, aliases, use of history and errors, which are involved in the dataset.

Table 16: Data report (insert_report) database table schema

\begin{tabular}{|l|l|}
\hline Field Name & Description \\
\hline id & This field contains the report id. \\
\hline filename & This field indicates the filename for \\
& which this report was generated. \\
\hline uid & This field is used to indicate the \\
\hline
\end{tabular}




\begin{tabular}{|l|l|}
\hline commands & user that this report belongs to. \\
& This field indicates the number of \\
& command lines that were executed \\
& by the given user. \\
\hline history & This field indicates the number of \\
& times the user resorted to using its \\
& history database. \\
\hline errors & This field indicates the number of \\
& times an error occurred while a user \\
& executed its command lines. \\
\hline aliases & This field indicates the number of \\
& times the user resorted to using an \\
& alias. \\
\hline lines & This field indicates the number of \\
& lines in the filename. \\
\hline
\end{tabular}

Primarily, the generated reports (Table 17) allow us to test the integrity of the database by comparing the results to its raw counterparts. The general statistical understanding of the dataset will also allow us to plan our implementation in a more meaningful way.

Our new knowledge of the data allows us to make better choices for the future. For example, based on this, we can determine which users will be beneficial for our testing purposes. Hence, if a user does not have sufficient amount of data items, then it 
becomes difficult to process a complete set of tasks in most training environments. The reports will also be helpful to index users who have made use of their history, aliases or are disposed to make errors in their command lines or vice versa. This type of information can become crucial in many research related tasks, particularly tasks that involve datasets being used in a controlled environment. The generated statistics for the Greenberg's dataset is made available in Appendix A: Generated Statistics for the Greenberg Dataset (Ordered by Commands).

Table 17: Sample reports in the 'insert_report' table from 5 different users

\begin{tabular}{|l|l|l|l|l|l|l|l|}
\hline id & filename & uid & commands & history & errors & aliases & lines \\
\hline 1 & scientist 1 & 1 & 1856 & 54 & 111 & 761 & 11792 \\
\hline 2 & scientist-10 & 2 & 2024 & 77 & 120 & 730 & 12658 \\
\hline 3 & scientist-11 & 3 & 205 & 0 & 13 & 0 & 1380 \\
\hline 4 & scientist-12 & 4 & 2499 & 53 & 52 & 1162 & 15412 \\
\hline 5 & scientist-13 & 5 & 3593 & 357 & 118 & 204 & 21988 \\
\hline
\end{tabular}

\subsubsection{Naïve Bayes Classifier}

The Naïve Bayes classifier is used as a learning mechanism (Detector box of Figure 1) in order to understand the available sample data.

The sample data is used in order to train the system and to familiarize it with possible outcomes. In principle, the available sample dataset will determine our expectations in anticipating accurate results in detecting legitimate versus illegitimate 
user sessions. The quality and the scale of available training data to the system will dictate our confidence in its results.

The basic algorithm involved in recognizing different predefined classifications with the use of the Naïve Bayes classifier involves two procedures, namely the Naïve Bayes learning mechanism and the Naïve Bayes classification. We first train our classifier with the available training data by using the Naïve Bayes learning mechanism. Once our learning procedure is completed, we classify new sets of input using our trained system.

In order to proceed with the learning mechanism, we first determine certain attributes that directly influence the Naïve Bayes learning algorithm. As previously stated, the Naïve Bayes classifier is typically used to classify text documents such as electronic news articles $[44 ; 45]$ or to classify spam, websites, documents, to name a few [23; 24]. But in the case of intrusion detection and profiling of legitimate users (that we deal with in this thesis), the classification must be achieved differently.

Here, our approach for classification consists in considering the possible outcomes of an IDS, i.e. the detection of a masquerader (illegitimate user) or the detection of a legitimate user. Therefore, we determine that our target value is either legitimate or illegitimate. Based on our training data, we can then determine the characteristics of a legitimate user. Yet, we do not have a direct understanding of what characterizes an illegitimate user. Naturally if a legitimate user is not detected, then the user must be considered as illegitimate. However the Naïve Bayes classifier requires us to have certain understanding on all defined classifications in our dataset 
prior to the detection. The absence of the training data for illegitimate users prevents us from gaining any understanding on the behavioural tendencies of a potential masquerader. Therefore, due to this shortcoming, we are obliged to practice the common adaptation [10] of using any training data available to us that does not belong to the potential legitimate user and consider this as the training data for an illegitimate user. Certainly, such an assumption may have certain consequences that may skew the final classification results. Depending on the scale of the dataset, an immediate consequence based on this assumption is as follows. Due to the nature of the Naïve Bayes classifier algorithm, the number of incidences in any classification is of importance. For instance, in our dataset of 168 users, the available training data for one legitimate user compared to its illegitimate counterpart (167 users) can potentially disrupt the classification. The reasoning behind this claim lies solely in the nature of the Naïve Bayes classifier algorithm.

Once the target values for the classification have been decided, we need to traverse through the training data and identify each element as a member of each target value. We then introduce the set $V$ to represent all the possible target values $v_{j}$. In order to begin the learning process we also introduce the setVocabulary. This set includes all the distinct words (command lines) that are available within the training data. The Vocabulary set can be regarded as our dataset dictionary.

Two probability terms $P\left(v_{j}\right)$ and $P\left(w_{k} \mid v_{j}\right)$ are used as the driving forces of the learning mechanism within the Naïve Bayes classifier. The term $P\left(v_{j}\right)$ also known as the prior probability, represents the probability of the target value $v_{j}$ occurring within 
the available training data. The term $P\left(w_{k} \mid v_{j}\right)$ represents the conditional probability, that a randomly selected word (command-line) from the training data belonging to the target value $v_{j}$ will be the word $w_{k}$.

Once learning is completed, we can then classify new sets of input using the following Equation:

$$
V_{N B}=\operatorname{argmax}_{v_{j} \in V} P\left(v_{j}\right) \prod_{i \in \text { positions }} P\left(a_{i} \mid v_{j}\right)
$$

The procedural steps that are required to train (hence produce the profile of a user) and classify user command sequences using the Naïve Bayes classifier are discussed next.

\subsubsection{Profiling Users}

In order to test the legitimacy of a user session, we must first develop an understanding of what constitutes a legitimate user session. To this effect, it is required to make use of the available training data which corresponds to the normal working state of any particular user within the system, then, develop a profile that accurately represents such user. This can be achieved by using a Naïve Bayes learning mechanism. The detailed procedural steps involved in implementing our Naïve Bayes learning mechanism with our dataset, in order to create a set of independent and distinct user profiles, is described as follows.

Our target value set $V$ is defined as as:

$$
V=\{\text { legitimate, } \text { illegitimate }\}
$$


As previously mentioned, the Naïve Bayes classifier requires the evaluation of the two probability terms $P\left(v_{j}\right)$ and $P\left(w_{k} \mid v_{j}\right)$ with regards to the training data, in order to successfully classify the new input.

To calculate $P\left(v_{j}\right)$ with respect to our new target value set $V$, we evaluate the following:

$$
\begin{gathered}
\mid \text { data }\left|=\sum_{j \in V}\right| \text { data }_{j} \mid \\
=\mid \text { data }_{\text {legitimate }}|+| \text { data }_{\text {illegitimate }} \mid
\end{gathered}
$$

where data is our training data.

$$
\begin{aligned}
P\left(v_{\text {legitimate }}\right)=\frac{\mid \text { data }_{\text {legitimate }} \mid}{\mid \text { data } \mid} & \\
P\left(v_{\text {illegitimate }}\right) & =\frac{\mid \text { data } \text { illegitimate }_{\text {lata }} \mid}{\mid \text { dat }} \\
& =1-P\left(v_{\text {legitimate }}\right)
\end{aligned}
$$

We estimate the conditional probability $P\left(w_{k} \mid v_{j}\right)$ the same way as done in [20], i.e.

$$
P\left(w_{k} \mid v_{j}\right)=\frac{n_{k}+1}{n+\mid \text { Vocabulary } \mid}
$$

where $w_{k} \in$ Vocabulary,

$n_{k}$ is the number of times the command line $w_{k}$ occurs in data $a_{j}$ and $n$ is the total number of distinct command lines in data $_{j}$ 
Having the preceding algorithms outlined, we can begin to document and profile every user within our training dataset.

In order to implement a successful training session, it is required to have a sufficient amount of data for each user's profile. Lack of sufficient data will directly contribute to inaccurate results. Our initial confidence in the system relies on the quality and the availability of a rich representation of a user's behavioural tendencies in a form of a dataset. By investigating the general statistical information (See Appendix A) regarding the available dataset, we can make certain decisions in regards to possible usability and suitability for each user's data and their potential candidacy for our training sessions.

Retrieving the generated statistical information allows us to consider each user as a potential candidate for a training session. As a rule of thumb, we consider each user that has equal or greater than 1500 command lines in its data pool as such candidate. After applying this rule to our 168 user dataset, we witness that 75 of the users meet the requirements.

These 75 users will be denoted as victims. We then use the available data associated with each victim to build our profiles. To this effect, we have considered the first 1000 command lines of each victim as the source for our training data. Once the victims have been identified and their designated training data has been extracted, we proceed to gain a more detailed understanding on the overall commuted training data. Based on the final commuted training data, a vocabulary is built, which consists of all 
distinct command lines used by all victims along with their number of occurrences. This vocabulary is used as a reference to build each victim's profile.

Along with the vocabulary, each user's distinct command lines are identified and recorded in a separate table. This table contains all the distinct command lines witnessed in the training data that belong to each user, along with their number of occurrences.

In order to build a profile for every user, all command lines witnessed in the vocabulary are coupled with each user. A user profile consists of all the terms (command-lines) within the vocabulary along with the probabilities $p_{\text {legitimate }}$ and $p_{\text {illegitamate }}$ associated with the term with respect to the user. These probabilities are the representations of the results gained from evaluating the probability term $P\left(w_{k} \mid v_{j}\right)$, where $w_{k}$ represents each command line in the vocabulary. $p_{\text {legitimate }}$ indicates the probability that the given command line belongs to the user, where $p_{\text {illegitimate }}$ indicates the probability that the given command line belongs to other users within the training data.

The vocabulary associated with our training data consisted of 17,982 terms (command lines). Therefore, each user's profile consists of the same number of terms along with their associated probabilities. After the completion of the learning mechanism, 1,348,650 records were generated, representing the profile information for the 75 victims. The following table (Table 18) represents a small segment of a profile belonging to one of the victims. 
Table 18: Small segment of a given profile

\begin{tabular}{|l|l|l|l|}
\hline uid & Command Line & \multicolumn{1}{|c|}{$\boldsymbol{p}_{\text {legitimate }}$} & \multicolumn{1}{c|}{$\boldsymbol{p}_{\text {illegitimate }}$} \\
\hline 78 & $\mathrm{ls}$ & 0.40874959414524 & 0.43698392003477 \\
\hline 78 & $\mathrm{fg}$ & 0.12359203459912 & 0.12143113580107 \\
\hline 78 & $\mathrm{e}$ & 0.11171047003469 & 0.052685310845613 \\
\hline 78 & lpq & 0.092699966731619 & 0.046794399410464 \\
\hline 78 & bye & 0.059431585951238 & 0.023954223940219 \\
\hline 78 & myada & 0.052302647212585 & $5.5574636180644 \mathrm{e}-07$ \\
\hline 78 & e conq.a & 0.052302647212585 & $5.5574636180644 \mathrm{e}-07$ \\
\hline 78 & ada -m conq.a & 0.049926334299701 & $5.5574636180644 \mathrm{e}-07$ \\
\hline 78 & a.out & 0.042797395561047 & 0.018062312505071 \\
\hline 78 & who & 0.03329214390951 & 0.040570040158232 \\
\hline 78 & purge & 0.03329214390951 & 0.0016122201956005 \\
\hline 78 & rwho | more & 0.035668456822394 & 0.0043909520046327 \\
\hline 78 & e queens.a & 0.016657953519319 & $5.5574636180644 \mathrm{e}-07$ \\
\hline
\end{tabular}

In Table 18, each command line is represented with its associated $p_{\text {legitimate }}$ and $p_{\text {illegitimate. }}$ In the next section, we discuss the classification process involved in determining whether a command line is classified as legitimate or illegitimate.

\subsubsection{Classifying Users}

Once we have established the probability values $p_{\text {legitimate }}$ and $p_{\text {illegitimate }}$ for all the terms within the vocabulary with respect to each victim, we can proceed to classify new terms (command-lines). As previously mentioned, ideally $p_{\text {illegitimate }}$ should represent the probability that the command line belongs to masqueraders. However, due to the absence of such data, it is required to build the probability from other sources. Although it may seem that the data is not authentic, it will nevertheless give a fair representation of what a legitimate user is not, which is the sole purpose of creating the counterpart classification to the target value legitimate. As a result of this assumption, 
$P\left(v_{\text {illegitimate }}\right)$ will always dominate $P\left(v_{\text {legitimate }}\right)$, since the available data ratio is $74: 1$. Consequently, the probability term $P\left(v_{j}\right)$ will dominate the Naïve Bayes classification, which in turn, will always classify inputs as illegitimate. In order to compensate for the dominating factor of the probability term $P\left(v_{\text {illegitimate }}\right)$, we have to make the assumption that the likelihood of a masquerader will be equal to that of the legitimate user (victim). Thus, the consideration of the term $P\left(v_{j}\right)$ can be eliminated from the classification process.

As an example, given the sequence of command lines described in the set $\{l s, c d$ classes, $c d$ cps511, pico deadlines, exit $\}$, the Naïve Bayes classifier will determine the classification based on each command-line's predetermined probability. For instance, if the probability distribution for an arbitrary user who has entered the command-lines within the given set is captured in Table 19, the Naïve Bayes classifier can be used to classify the set as whether it is legitimate or illegitimate compared to the given sample user profile. The Naïve Bayes classifier makes the assumption that each element in the set is independent, which explains the naïve nature of the classifier.

Table 19: Probability distribution for an arbitrary user

\begin{tabular}{|l|l|l|}
\hline Command Line & $\boldsymbol{p}_{\text {legitimate }}$ & $\boldsymbol{p}_{\text {illegitimate }}$ \\
\hline ls & 0.3121 & 0.4351 \\
\hline cd classes & 0.3123 & 0.0922 \\
\hline cd cps511 & 0.0422 & 0.0311 \\
\hline pico deadlines & 0.0911 & 0.0022 \\
\hline exit & 0.0332 & 0.0021 \\
\hline
\end{tabular}

In this example, to determine the classification, we perform the following calculations: 


$$
\begin{gathered}
V_{N B}=\operatorname{argmax}_{v_{j} \in V} \prod_{i \in \text { positions }} P\left(a_{i} \mid v_{j}\right) \quad(10) \\
\prod_{\mathrm{i} \in \text { positions }} \mathrm{P}\left(\mathrm{a}_{\mathrm{i}} \mid \mathrm{v}_{\text {legitimate }}\right)=\mathrm{P}\left(\mathrm{ls} \mid \mathrm{v}_{\text {legitimate }}\right) \mathrm{P}\left(\text { cd classes } \mid \mathrm{v}_{\text {legitimate }}\right) \ldots \mathrm{P}\left(\text { exit } \mid \mathrm{v}_{\text {legitimate }}\right) \\
=(0.3121)(0.3123)(0.0422)(0.0911)(0.0332) \\
=1.24 \times 10^{-5} \\
\prod_{\mathrm{i} \in \text { positions } \quad \mathrm{P}\left(\mathrm{a}_{\mathrm{i}} \mid \mathrm{v}_{\text {illegitimate }}\right)}=\mathrm{P}\left(\mathrm{ls} \mid \mathrm{v}_{\text {illegitimate }}\right) \mathrm{P}\left(\text { cd classes } \mid \mathrm{v}_{\text {illegitimate }}\right) \ldots \mathrm{P}\left(\text { exit } \mid \mathrm{v}_{\text {illegitimate }}\right) \\
=(0.4351)(0.0922)(0.0311)(0.0022)(0.0021) \\
=5.76 \times 10^{-9}
\end{gathered}
$$

Since $5.76 \times 10^{-9}<1.24 \times 10^{-5}$, it is concluded that the set is classified as legitimate, i.e. the given set is recognized as legitimate input produced by the owner of our sample user profile.

In our implementation, we use the Naïve Bayes classifier to classify each new input that is witnessed in our evaluation. However, the final decision to accept or reject a user is made by using the sequential sampling method, as described in the next section.

\subsubsection{Continuous Authentication and the Sequential Sampling Technique}

In order to appropriately evaluate the legitimacy of a particular user against a certain profile, an evaluation technique is required, where the crucial characteristics of the system are recognized. In an IDS, decisions can be made using any number of input values, regardless of the relevancy of the data in question. However, the accuracy of the decisions can be questionable. In order to maintain a certain confidence rate in our 
system, we have adopted a recently proposed evaluation technique [1], which can be considered as a pioneer method for achieving continuous authentication based on learning biometrics data. In this thesis, this technique has been adapted for use in the case of user command sequence.

The term continuous authentication $[46 ; 47 ; 48]$ refers to a system where the authentication process is continuously active throughout the session. Typically, the behavioural patterns of the user are tested and evaluated against a predefined signature. The data rates for the input streams are unknown. Hence, the authenticity of the user is tested continuously as data becomes available.

Due to the nature of our application, the input data flow rate is also unknown; hence an evaluation technique is required that can systematically adapt itself to the rate at which new input data are available. In order to evaluate the legitimacy of a user, we use the above-mentioned evaluation technique (sequential sampling technique [1]). This method has previously been used to evaluate the legitimacy of a user based on mouse dynamics. This is the first time that the method is being used for evaluating command line sequences.

The sequential sampling technique is a dynamic sample size decision technique. Typically, a classical sampling is used to make the decision on the validity of a particular user [1]. In classical sampling, the sample size is predetermined and decisions are only made when the end of the data collection procedure is met. The sequential sampling method was introduced to compensate for certain shortcomings of the classical sampling approach when dealing with continuous authentication. In a 
classical sampling approach, a decision cannot be made, until there is a sufficient amount of data available. Hence, the system can be vulnerable during that time, and thus the system's TTA (Time-to-Alarm) can be significantly influenced by such a method. By using the sequential sampling method, the decision making is active during data collection. Therefore, decisions can be made as new input is presented, and data collection and analysis can be done simultaneously [1].

In order to utilize the sequential sampling technique, it is required to first model a sampling plan which consists of three regions, namely, Accept, Reject and Continue. While the data is being collected continuously (as shown in Figure 3), the sample size is incremented accordingly until a decision is made.

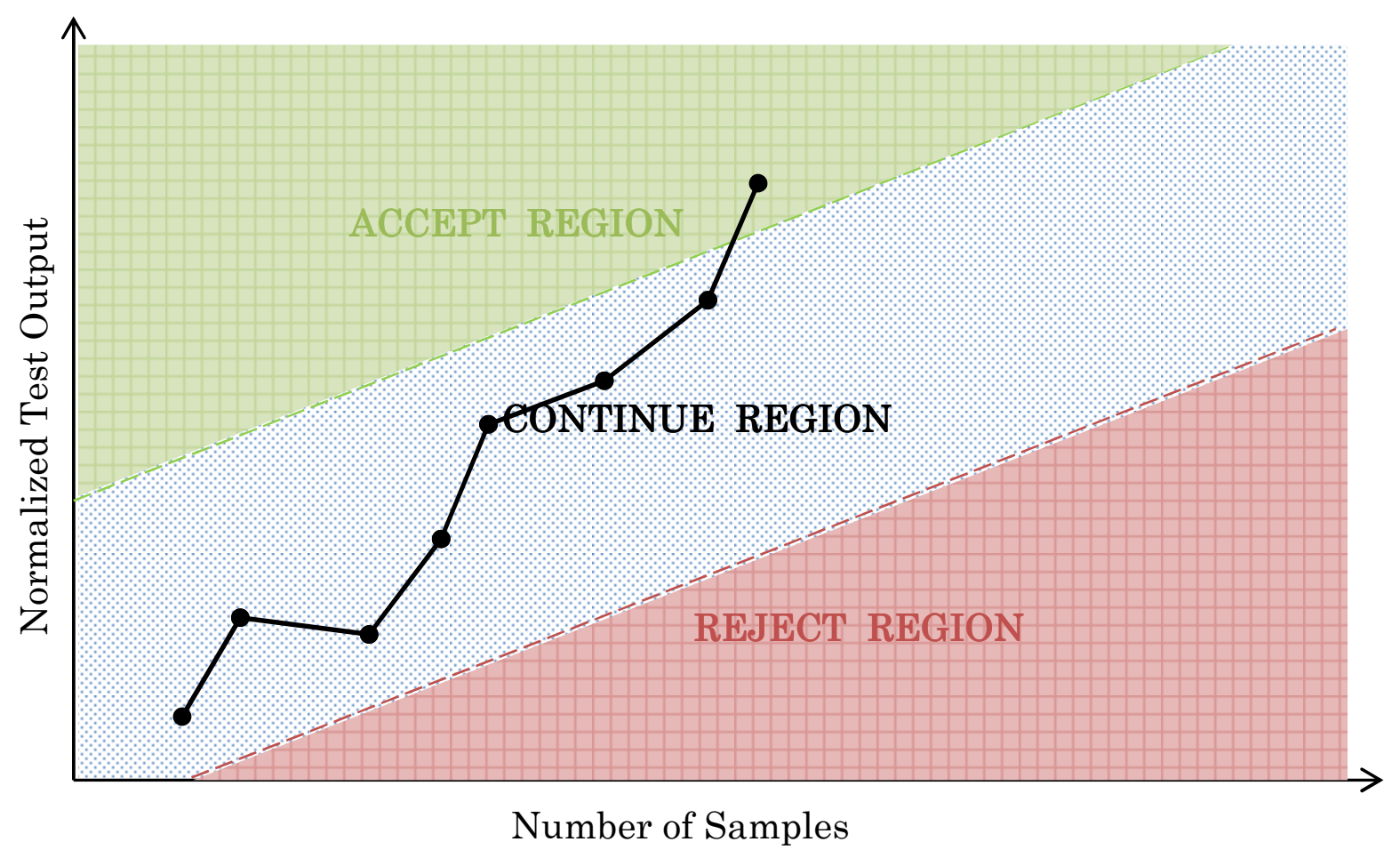

Figure 3: General sampling plan 
The sequential sampling technique will continuously test the null hypothesis as the number of test inputs increases. The amount of collected data within each iteration is based on a predetermined sample size, while the sample size itself is determined based on the application in which the sequential sampling technique is used. Depending on the application the sample size can range from collecting only a single data item to collecting a large set of data items.

The sampling plan is developed in accordance with the following parameters [1]:

$$
\begin{aligned}
& \alpha=\text { acceptable type I error (false positive) } \\
& \beta=\text { acceptable type II error (false negative) } \\
& p_{1}=\text { lower threshold limit (as proportion) } \\
& p_{2}=\text { higher threshold limit (as proportion) }
\end{aligned}
$$

A decision is only made when a normalized confidence rate value enters one of the decision regions (i.e. accept or reject). The acceptance and rejection lines are expressed as follows [1]:

$$
\begin{aligned}
& N_{C R}=h_{2}+s n \\
& N_{C R}=-h_{1}+s n
\end{aligned}
$$

where $N_{C R}$ is the normalized value of the confidence ratio computed for test number $n$.

$$
N_{C R}=C R_{n} \times \frac{n}{100}
$$

The parameters $h_{1}, h_{2}$ and $s$ can be expressed as follows: 


$$
\begin{aligned}
& h_{1}=\ln \frac{1-\alpha}{\beta} \div \ln \frac{p_{2}\left(1-p_{1}\right)}{p_{1}\left(1-p_{2}\right)} \\
& h_{2}=\ln \frac{1-\beta}{\alpha} \div \ln \frac{p_{2}\left(1-p_{1}\right)}{p_{1}\left(1-p_{2}\right)} \\
& s=\ln \frac{1-p_{1}}{1-p_{2}} \div \ln \frac{p_{2}\left(1-p_{1}\right)}{p_{1}\left(1-p_{2}\right)}
\end{aligned}
$$

It can be observed that equation 13 and 14, follow the basic principles of a straight line. Therefore, the variables $h_{1}$ and $h_{2}$ influence the distance between the two lines and $s$ represents their slope [1]. 


\section{Chapter 4: Experimental Evaluation}

This Chapter discusses the performance evaluation of the Continuous Authentication Based on Learning User Command Sequence (CABLUCS) scheme proposed in this thesis. This includes the evaluation approach, the experiments setup and operational aspects, and finally a description of the results obtained.

\subsection{Challenges}

The goal of a IDS is to accurately determine the legitimacy of a given user in a timely fashion. To this effect, several parameters can been predefined and then used to measure the efficiency and accuracy of the system in terms of detection rate. In this thesis, we have considered the following parameters.

- The Detection Rate (DR): this is the rate at which the system can successfully detect an intrusion, in the event of a masquerade attack.

- False Reject Rate (FRR) and False Accept Rate (FAR): Typically, two types of errors arose when decisions are made using experimental data in an IDS. They are symbolized as Type I and Type II errors, respectively

- A Type I error implies that a reject decision has inappropriately been made, indicating that a false rejection has occurred. In this case, the rejection of a legitimate user has happened. The FRR represents the percentage in which the system has falsely rejected a legitimate user.

- A Type II error implies that an accept decision has inappropriately been made. This type of error indicates that a false acceptance has occurred. In this case, the acceptance of a masquerader as a legitimate user has happened. The FAR represents the percentage in which the system has falsely accepted a 
masquerader as a legitimate user. It is also an indication of the rate at which the system has been compromised.

- Time-to-Alarm (TTA) and Mean-Time-to-Alarm (MTTA): The accuracy of the detection mechanism is an important factor. However, if the decision is not made in a timely fashion, the system can be jeopardised. The TTA indicates the time elapsed until the masquerader was detected and the MTTA represents its responsiveness.

In the design of an IDS, attempting to minimize the FRR, FAR, and the MTTA rates is a difficult task in the sense that these attributes are loosely related to each other. In order to reduce the MTTA value, decisions must be made faster. However, a quick decision may not be appropriate since this may lead to increased FAR and FRR. The challenge is thus to minimize all attributes while maintaining an efficient and operational system.

\subsection{Evaluation Approach and Setup}

In order to setup the working environment for our experiments, we have acquired the LAMP software bundle. LAMP was installed on a Dell Workstation, Quad Xeon Processors at $1.86 \mathrm{GHz}$ with $8 \mathrm{~GB}$ RAM. LAMP is an open source software bundle that consists of Linux, Apache HTTP Server, MySQL relational database management system and PHP. This combination is generally used to create dynamic, database driven, web applications. LAMP projects tend to be platform independent, hence once developed, they can be executed on most operating systems. 
PHP is a scripting language with syntax similar to the $\mathrm{C}$ programming language, which is simple to deploy and execute. It is generally bundled for the use of dynamic web programming in relations with MySQL. The setup was configured to be sensitive to errors, while disabling caching and timeouts. Caching is typically used in PHP for the purpose of enhancing the processing time for the re-runs of the same code. Because we are interested in monitoring and differentiating the processing time (in seconds) of the different test cases, we have set this feature to 'disabled'. PHP processing timeouts are usually set to 30 seconds, which is a reasonable time if we are concerned with executing a program that results in producing a simple web page. However, due to the nature of our experiment, we anticipate a much higher of processing time. Therefore, timeouts have also been disabled. In our experiments, we have also configured PHP warning and error settings, to make all potential warnings and errors visible within our apparatus.

Along with PHP, the MySQL server and the Apache HTTP Server also utilize caching and other performance enhancing features as the system adapts itself to its environment. Therefore, for every test case, the servers are reset to their original status and restarted accordingly. As a result, certain tests could not be done simultaneously and longer testing times are required to test different parameters. This procedure is followed to maintain a fair comparison between the different test cases in comparative processing times (measured in seconds). These precautionary steps do not influence the FRR, FAR and MTTA values since these values do not incorporate time (in seconds) as their unit. The MTTA value is based on the number of actions required to make a decision, thus is not based on the processing time (in seconds) since different 
workstations can produce different processing times while testing the same IDS, but in contrast, they will all output the same MTTA value.

\subsubsection{Extracting the Data from Its Raw Format}

A PHP script was written in order to extract the data from its raw format into a MySQL database. The script traverses through the different folders looking for files that matched the required criteria. The criteria are set based on the provided Greenberg dataset structure. Once the script has determined that a file meets the criteria, it detects the user and its type. Using the discovered information regarding the user, the script then creates a user record in the database, while documenting the related information.

Once the user has been determined, the script traverses through its given data, scanning for user sessions. Each user session that is found is recorded in the database. The related information on this user is documented, which include the session's start and end time. A function is then used to convert the start and end time to a UNIX timestamp value.

\subsubsection{Extracting the Command Line of a Session}

Once a session has been determined, the data collection proceeds to extract command lines relative to the given session. Each command line is extracted from the session and is given an order number, which represents the order in which the command line is seen within that session. 
Certain string values (i.e. command-line, working directory and alias) are required to be character-escaped in order to meet certain PHP and MySQL compatibility issues. Due to the nature of the Greenberg dataset, each command line is known to be coupled with certain attributes (such as working directory, history, alias and error). In order to retrieve the attributes with respect to the command line, the script is parsed through each item and the necessary information is collected. An audit is also kept on the general statistical information of each user (i.e. the number of command lines, the errors, to name a few).

Due to certain hidden characters within the Greenberg dataset, several string comparisons in each user session tended to fail. By trimming whitespaces and other unknown hidden characters, this issue has been fixed. Binary data comparison is used in all tests, in order to represent a perfect match. The script finally traverses through every session within every 168 users available in the Greenberg dataset and records their entire data, while maintaining complete data integrity. The resulting data in its new format is highly accessible, easy to use, flexible and customizable.

\subsubsection{Deciding the Victims and Masqueraders}

After the above-mentioned data extraction, 31 masqueraders and 75 victims are decided by examining the general statistical information acquired. A PHP script is written in order to train the 75 profiles using our Naïve Bayes learning algorithm. The profiling process took approximately 5 hours to complete, yielding a total of 1,348,650 data items. 


\subsubsection{Calculating the Confidence Ratio}

In order to calculate the confidence ratio (CR), a set of command lines are tested against a given profile. For instance, considering the following test input set of five command-lines $\{l s, c d$ classes, vi hello.txt,whoami, exit $\}$, we test each command line against user $x$ 's profile. Assuming that after each command line's independent classification based on the Naïve Bayes classifier, 4 command lines are classified as legitimate and 1 is classified as illegitimate. In this example, our CR for the five

command lines belonging to user $x$ is obtained as $\frac{4}{5} \times 100=80$. Although initially it may seem that the tested input set belongs to user $x$ given a $\mathrm{CR}$ of $80 \%$, this conclusion is based on only five command lines. If the next five command lines produces a CR of $5 \%$, we can immediately sense that our original hypothesis may be faulty.

\subsubsection{Determining the Legitimacy of Users}

We use the sequential sampling technique in order to complement the nature of continuous authentication systems. The sequential sampling technique allows us to make better decisions as the size of our input set increases based on a given sample size. Different applications require different sample sizes.

The sequential sampling technique has been used to determine the legitimacy of users based on mouse dynamics, where sample sizes ranged from 25 to 100 [1]. The sample size used in a mouse dynamics application differs from that of a command-line based application. For instance, five command lines may differ in significance than five mouse gestures or clicks. 
In order to use the sequential sampling technique, we have built different sampling plans that will allow us to test the legitimacy of a given user. To build a sampling plan, we have to determine the accept, continue and reject regions. In order to determine these regions, we have established the parameters involved in producing the two lines that separate the three regions. Depending on the application, the values of the required parameters are different. In order to determine suitable values for the required parameters $p_{1}$ and $p_{2}$, which represent the lower and higher thresholds respectively, we have conducted a simple test. This test consisted in determining values that will adjust the distance between the accept/reject lines in such a way that it will satisfy the overall range of our CR values. After several trials and error cases, three sampling plans are selected. The values chosen for each sampling plan are recorded in Table 20.

Table 20: Three selected sampling plans

\begin{tabular}{|c|c|c|c|c|}
\hline $\begin{array}{c}\text { Sampling } \\
\text { Plan }\end{array}$ & $\boldsymbol{p}_{\mathbf{1}}$ & $\boldsymbol{p}_{\boldsymbol{2}}$ & $\boldsymbol{\alpha}$ & $\boldsymbol{\beta}$ \\
\hline A & 0.29 & 0.71 & 0.01 & 0.01 \\
\hline B & 0.30 & 0.70 & 0.01 & 0.01 \\
\hline C & 0.31 & 0.69 & 0.01 & 0.01 \\
\hline
\end{tabular}

In Table 20, $\alpha$ and $\beta$ represent the acceptable type I and the acceptable type II errors respectively. In all our test cases, the values for these two parameters are set to 0.01 . The three selected sampling plans are illustrated in Figure 4, Figure 5 and Figure 6. 


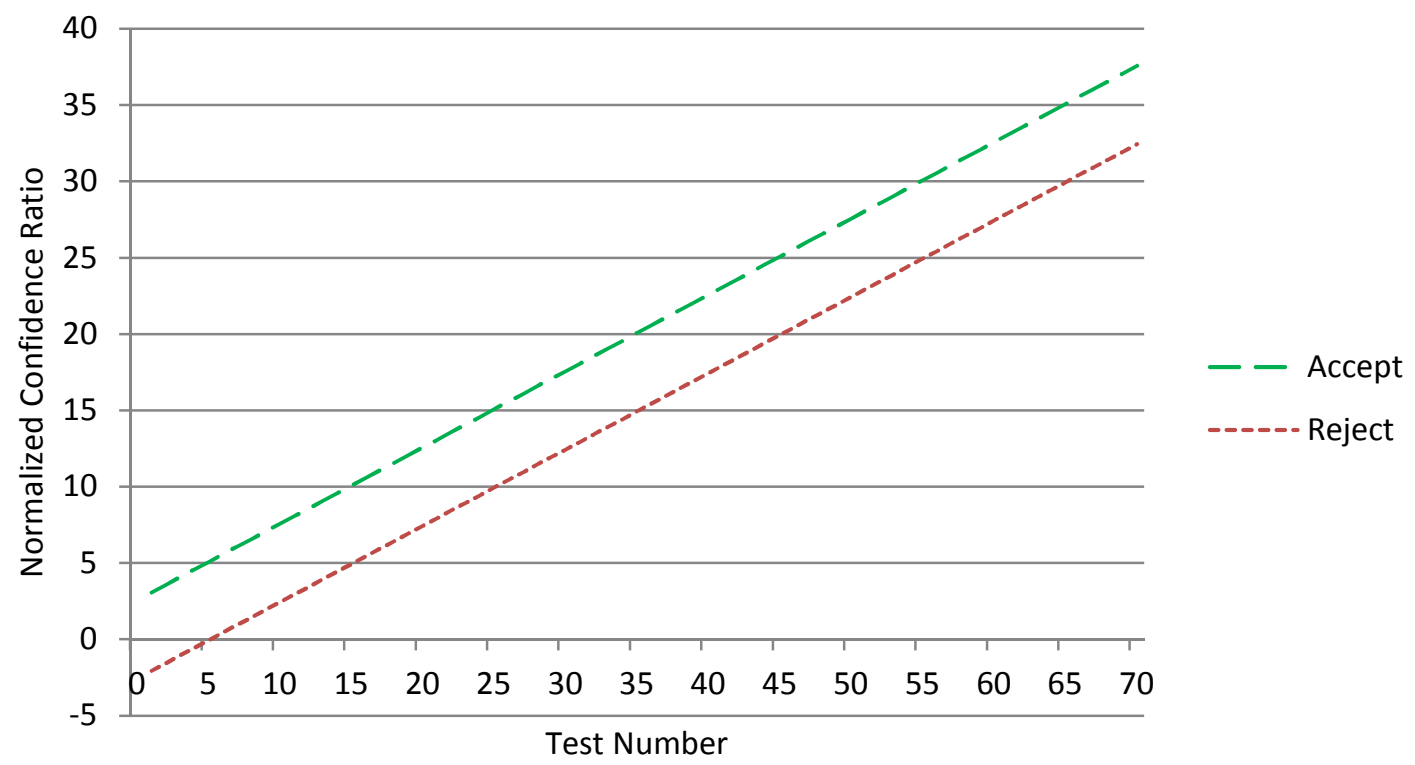

Figure 4: Sampling Plan $A\left(p_{1}=0.29, p_{2}=0.71, \alpha=0.01, \beta=0.01\right)$.

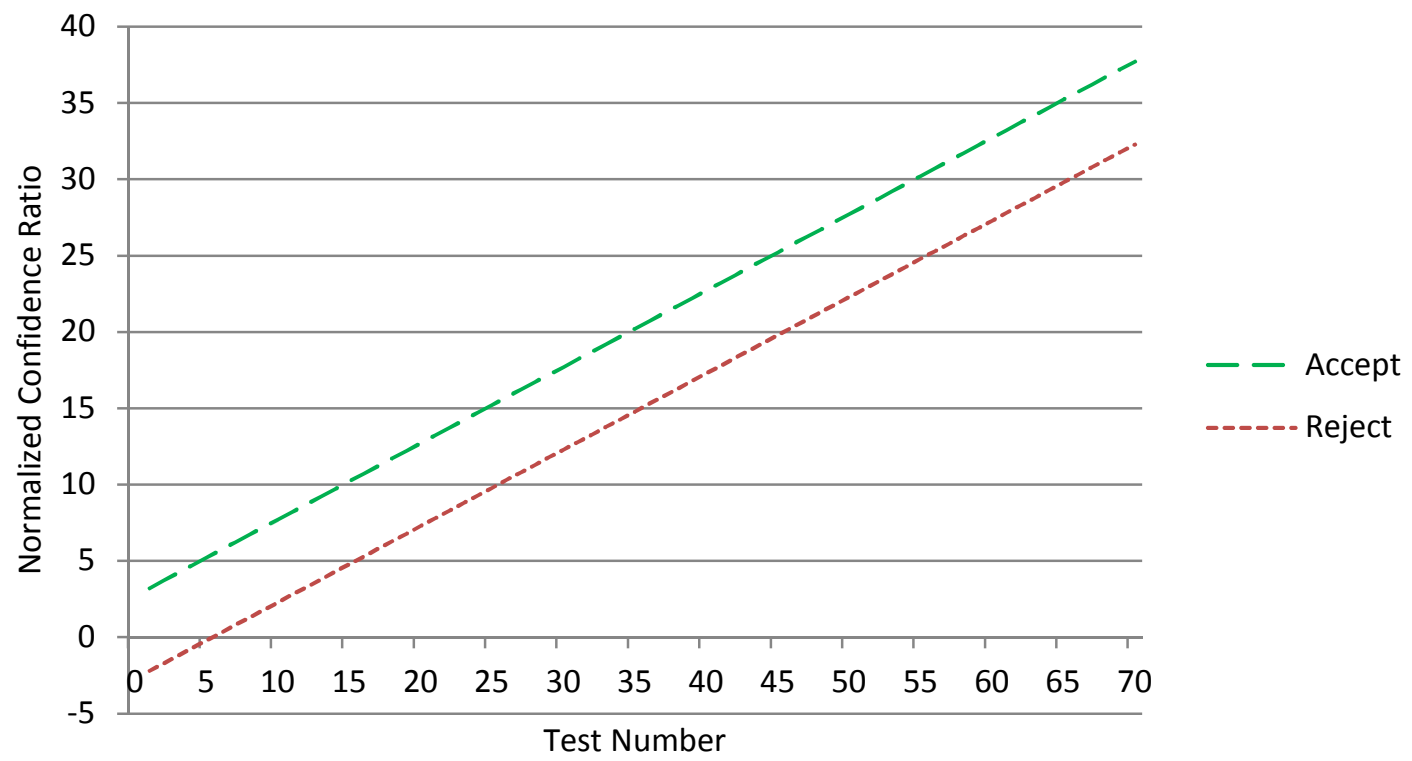

Figure 5: Sampling Plan B ( $\left.p_{1}=0.30 p_{2}=0.70, \alpha=0.01, \beta=0.01\right)$. 


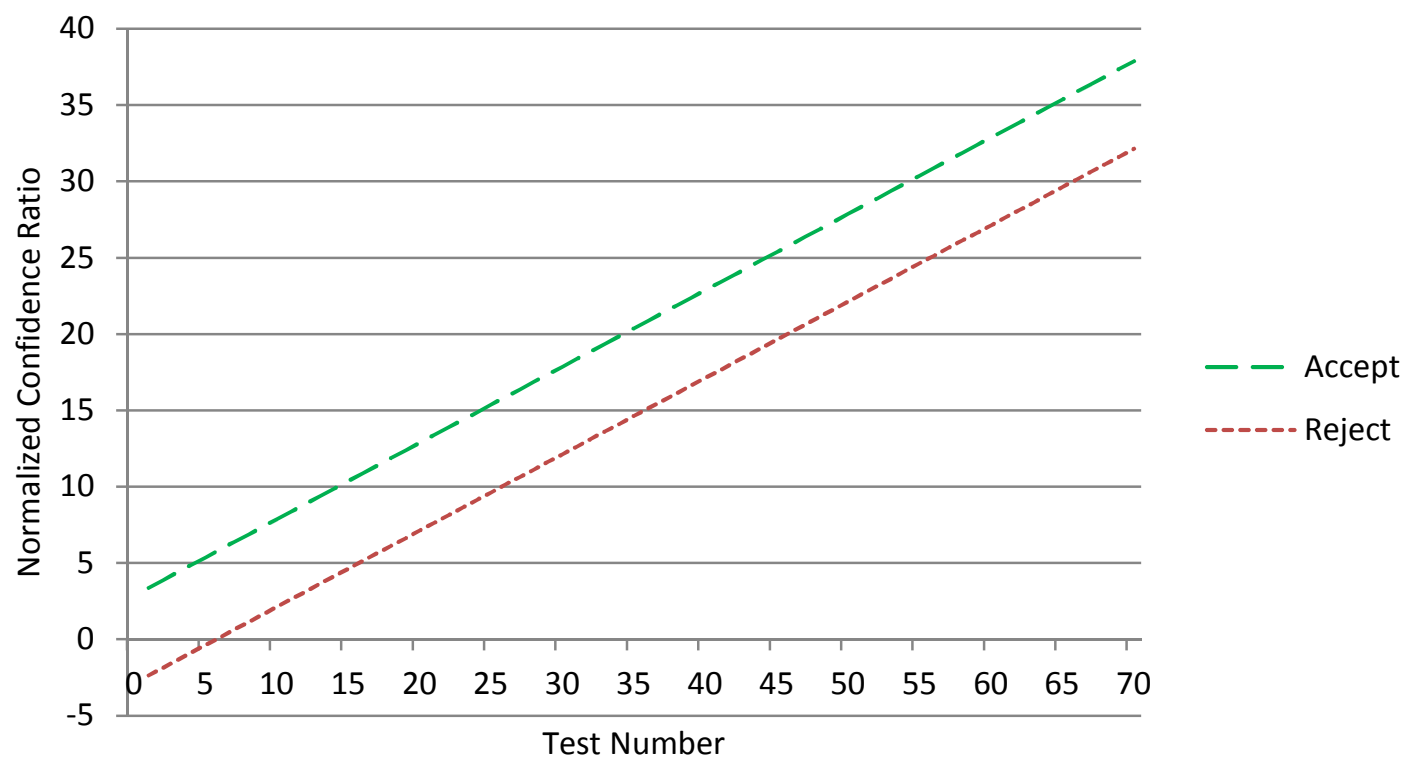

Figure 6: Sampling Plan $C\left(p_{1}=0.31 p_{2}=0.69, \alpha=0.01, \beta=0.01\right)$

\subsubsection{Calculating the MTTA, FAR, and FRR}

The $p_{1}$ and $p_{2}$ values determine the sensitivity of the system to decision making. As $p_{1}$ is decremented, the continue region becomes smaller and the system becomes more susceptible to making faster decisions. Hence, Sampling Plan A is expected to have a lower Mean-Time-to-Alarm (MTTA) value than Sampling Plan B and C. As $p_{1}$ is incremented, the MTTA value is expected to climb, hence, decisions are made slower. A lower MTTA does not always suggest a better system. The challenge is to lower the MTTA while making accurate decisions.

In order to calculate the False Rejection Rate (FRR), every victim is tested against its own profile. As previously mentioned, the first 1,000 command lines of a user are utilized in order to build its relative profile. It is crucial not to use the same 
data source as the new input for testing purposes; otherwise the results will not carry great weight in our conclusion. The first command-line used to test the user against its own profile is the $1,001^{\text {st }}$ element in the given user's data pool. There are 75 victims in total, hence, 75 tests are completed for each sampling plan in order to calculate the relative FRR.

Calculating the FAR value involves testing all masqueraders against all victims for each sampling plan. All 31 masqueraders are contributing in attacking each of the 75 victims. The expected results in this test are rejections. In the case where an acceptance has been issued to a masquerader, the result is recorded and the FAR value is updated accordingly.

A maximum TTA of 1000 command-lines is set in order to compensate for system halts. System halts can occur when a decision cannot be made using the available input data against the sampling plan. This can occur if the normalized confidence ratio continues to be in the continue region of the sampling plan without penetrating the final decision regions (i.e. accept or reject). System halts can also be triggered as a result of insufficient input data that can cause the prevention of a decision from being made.

\subsubsection{Comparing a User against a Given Profile}

In order to utilize the sequential sampling technique, for each sampling plan, a sample size must be set before any testing can begin. The sample size determines the number of command lines to sample before attempting to make a decision. For instance, a sample size of 5 means that the iterator will sample and accumulate every 5 command- 
lines as they are made available. Every iteration is recognized by a number, which is denoted as a test number. With a sample size of 5 command-lines, test number 3 indicates that 15 command lines have been collected and tested.

A PHP script is written in order to simulate the testing process of a user against a given profile. This script incorporates the sequential sampling technique in order to make decisions on the legitimacy of the test user. The three different sampling plans are tested using different sample sizes, while the results are recorded in two different MySQL tables. The engine_report MySQL table (Table 21) is used to detail the final results made by the algorithm as a user is tested against a profile.

Table 21: engine_report MySQL table schema

\begin{tabular}{|l|l|}
\hline Field Name & Description \\
\hline id & This field is used as the primary key \\
& for this table. It is used to identify \\
& the given report (record). \\
\hline uid_input & This field identifies the user that is \\
& being tested against a given profile. \\
\hline uid_profile & This field indicates the user-profile. \\
\hline iteration & This field indicates the number of \\
& iterations required to make a \\
& decision. \\
\hline decision_expected & This field indicates the expected \\
& decision to be made. (i.e. Accept if \\
\hline
\end{tabular}




\begin{tabular}{|c|c|}
\hline & uid_input is equal to uid_profile) \\
\hline decisioin_made & $\begin{array}{l}\text { is field indicates the final decision } \\
\text { tde after the testing was completed. }\end{array}$ \\
\hline seconds & $\begin{array}{l}\text { This field indicates the number of } \\
\text { seconds required to make a decision. }\end{array}$ \\
\hline sample_size & $\begin{array}{l}\text { This field indicates the sample size } \\
\text { used in the sequential sampling } \\
\text { technique. }\end{array}$ \\
\hline num_commands & $\begin{array}{l}\text { This field indicates the number of } \\
\text { command-lines that were required in } \\
\text { order to make a decision. }\end{array}$ \\
\hline num_trained_items & $\begin{array}{l}\text { This field indicates the number of } \\
\text { command-lines that were used to } \\
\text { train the profile. In all of the tested } \\
\text { cases, this number remained at } \\
1,000 \text {. }\end{array}$ \\
\hline p1 & $\begin{array}{l}\text { This field indicates the lower } \\
\text { threshold used in the sampling plan. }\end{array}$ \\
\hline $\mathrm{p} 2$ & $\begin{array}{l}\text { This field indicates the higher } \\
\text { threshold used in the sampling plan. }\end{array}$ \\
\hline alpha & $\begin{array}{l}\text { This field represents the acceptable } \\
\text { type I error (false positive) }\end{array}$ \\
\hline beta & This field represents the acceptable \\
\hline
\end{tabular}




\begin{tabular}{|l|l|}
\hline & type II error (false negative) \\
\hline max_iterations & This field indicates the maximum \\
& allowed number of iterations. \\
\hline
\end{tabular}

The details of each decision are recorded in the engine_output MySQL table (Table 22). This table outlined the successive progression of the decision making process of the sequential sampling technique (as shown in Table 23 and Table 24).

Table 22: engine_output MySQL table schema

\begin{tabular}{|c|c|}
\hline Field Name & Description \\
\hline report_id & $\begin{array}{l}\text { This field identifies the report that } \\
\text { this record belongs to. }\end{array}$ \\
\hline test_num & $\begin{array}{l}\text { This field indicates the test number } \\
\text { for the given iteration. (i.e. } 1,2,3 . . \text { ) }\end{array}$ \\
\hline accept_limit & $\begin{array}{l}\text { This field represents the accept } \\
\text { value, given the test number,p1, p2, } \\
\text { alpha and beta. }\end{array}$ \\
\hline reject_limit & $\begin{array}{l}\text { This field represents the reject value, } \\
\text { given the test number, } \mathrm{p} 1, \mathrm{p} 2 \text {, alpha } \\
\text { and beta. }\end{array}$ \\
\hline normalized_cr & $\begin{array}{l}\text { This field represents the normalized } \\
\text { confidence ratio after testing } n * N \\
\text { command lines, where } n \text { is the test }\end{array}$ \\
\hline
\end{tabular}




\begin{tabular}{|l|l|}
\hline number and $\mathrm{N}$ is the sample size. \\
\hline cr & This field represents the confidence \\
& ratio after testing $\mathrm{n}^{*} \mathrm{~N}$ command \\
& lines, where $\mathrm{n}$ is the test number and \\
& $\mathrm{N}$ is the sample size. \\
\hline num_commands & This field represents the number of \\
& command-lines used to calculate the \\
& confidence ratio. \\
\hline
\end{tabular}

Table 23: Details of the final results made by using CABLUCS

\begin{tabular}{|c|c|c|c|c|c|c|c|c|c|c|c|c|c|c|}
\hline Id & $\begin{array}{l}\text { Uid } \\
\text { Input }\end{array}$ & $\begin{array}{c}\text { Uid } \\
\text { Profile }\end{array}$ & Iteration & $\begin{array}{l}\text { Decision } \\
\text { Expected }\end{array}$ & $\begin{array}{l}\text { Decision } \\
\text { Made }\end{array}$ & Seconds & $\begin{array}{c}\text { Sample } \\
\text { Size }\end{array}$ & $\begin{array}{l}\text { Number of } \\
\text { Commands }\end{array}$ & $\begin{array}{l}\text { Number of } \\
\text { Trained } \\
\text { Commands }\end{array}$ & P1 & P2 & Alpha & Beta & Max Iteration \\
\hline 1 & 86 & 86 & 12 & Accept & Accept & 20 & 5 & 55 & 1000 & 0.3 & 0.7 & 0.01 & 0.01 & 1000 \\
\hline 2 & 30 & 30 & 67 & Accept & Reject & 138 & 5 & 330 & 1000 & 0.3 & 0.7 & 0.01 & 0.01 & 1000 \\
\hline 3 & 89 & 55 & 6 & Reject & Reject & 9 & 3 & 15 & 1000 & 0.31 & 0.69 & 0.01 & 0.01 & 1000 \\
\hline 4 & 24 & 24 & 9 & Accept & Accept & 77 & 20 & 160 & 1000 & 0.29 & 0.71 & 0.01 & 0.01 & 1000 \\
\hline
\end{tabular}

Table 24: Successive progression of the decision making process

\begin{tabular}{|c|c|c|c|c|c|c|}
\hline Report id & Test Number & Accept Limit & Reject Limit & Normalized CR & CR & Number of Commands \\
\hline 3 & 1 & 3.3715216902596 & -2.3715216902596 & 0 & 0 & 3 \\
\hline 3 & 2 & 3.8715216902596 & -1.8715216902596 & 0 & 0 & 0 \\
\hline 3 & 3 & 4.3715216902596 & -1.3715216902596 & 0 & 0 \\
\hline 3 & 4 & 4.8715216902596 & -0.8715216902596 & 0 & 0 \\
\hline 3 & 5 & 5.3715216902596 & -0.3715216902596 & 0 & 0 & 12 \\
\hline
\end{tabular}




\begin{tabular}{|l|l|l|l|l|l|l|l|}
\hline 3 & 6 & 5.8715216902596 & 0.12847830974036 & 0 & 0 \\
\hline
\end{tabular}

\subsection{Performance Results}

The following table describes the annotations used to describe each completed test.

Here, TID is used to indicate the given test. The list of prepared sampling plans and tests are shown in Table 25.

Table 25: List of prepared sampling plans and tests

\begin{tabular}{|c|c|c|c|c|c|c|}
\hline TID & $\begin{array}{c}\text { Sample } \\
\text { Size }\end{array}$ & $\begin{array}{c}\text { Sampling } \\
\text { Plan }\end{array}$ & $p_{1}$ & $p_{2}$ & $\alpha$ & $\boldsymbol{\beta}$ \\
\hline $1_{\mathrm{A}}$ & 3 & A & 0.29 & 0.71 & 0.01 & 0.01 \\
\hline $1_{R}$ & 3 & A & 0.29 & 0.71 & 0.01 & 0.01 \\
\hline $2_{\mathrm{A}}$ & 3 & B & 0.30 & 0.70 & 0.01 & 0.01 \\
\hline $22_{R}$ & 3 & B & 0.30 & 0.70 & 0.01 & 0.01 \\
\hline $3 \mathrm{~A}$ & 3 & $\mathrm{C}$ & 0.31 & 0.69 & 0.01 & 0.01 \\
\hline $3_{R}$ & 3 & B & 0.31 & 0.69 & 0.01 & 0.01 \\
\hline $4_{\mathrm{A}}$ & 3 & - & 0.32 & 0.68 & 0.01 & 0.01 \\
\hline $5_{\mathrm{A}}$ & 3 & - & 0.33 & 0.67 & 0.01 & 0.01 \\
\hline $6_{\mathrm{A}}$ & 3 & - & 0.34 & 0.66 & 0.01 & 0.01 \\
\hline $7 \mathrm{~A}$ & 3 & - & 0.35 & 0.65 & 0.01 & 0.01 \\
\hline $8 \mathrm{~A}$ & 5 & A & 0.29 & 0.71 & 0.01 & 0.01 \\
\hline $4 R$ & 5 & A & 0.29 & 0.71 & 0.01 & 0.01 \\
\hline $9 \mathrm{~A}$ & 5 & B & 0.30 & 0.70 & 0.01 & 0.01 \\
\hline $9_{R}$ & 5 & B & 0.30 & 0.70 & 0.01 & 0.01 \\
\hline $10_{\mathrm{A}}$ & 5 & $\mathrm{C}$ & 0.31 & 0.69 & 0.01 & 0.01 \\
\hline $10_{R}$ & 5 & $\mathrm{C}$ & 0.31 & 0.69 & 0.01 & 0.01 \\
\hline $11_{\mathrm{A}}$ & 10 & A & 0.29 & 0.71 & 0.01 & 0.01 \\
\hline $11_{R}$ & 10 & A & 0.29 & 0.71 & 0.01 & 0.01 \\
\hline $12_{\mathrm{A}}$ & 10 & B & 0.30 & 0.70 & 0.01 & 0.01 \\
\hline $12_{\mathrm{R}}$ & 10 & B & 0.30 & 0.70 & 0.01 & 0.01 \\
\hline $13_{\mathrm{A}}$ & 10 & $\mathrm{C}$ & 0.31 & 0.69 & 0.01 & 0.01 \\
\hline $13_{R}$ & 10 & $\mathrm{C}$ & 0.31 & 0.69 & 0.01 & 0.01 \\
\hline $14_{\mathrm{R}}$ & 15 & A & 0.29 & 0.71 & 0.01 & 0.01 \\
\hline $15_{R}$ & 15 & B & 0.30 & 0.70 & 0.01 & 0.01 \\
\hline $16_{R}$ & 15 & $\mathrm{C}$ & 0.31 & 0.69 & 0.01 & 0.01 \\
\hline $17_{\mathrm{R}}$ & 20 & A & 0.29 & 0.71 & 0.01 & 0.01 \\
\hline $18 \mathrm{R}$ & 20 & B & 0.30 & 0.70 & 0.01 & 0.01 \\
\hline $19_{R}$ & 20 & $\mathrm{C}$ & 0.31 & 0.69 & 0.01 & 0.01 \\
\hline
\end{tabular}


Figure 7 and Figure 8 illustrate a visualization of the sequential sampling technique using actual data taken from our experiment. Figure 7 shows a user's test input on its own profile based on TID 9 A TID values with the subscript letter $A$, represent a test case where the expected decision is an acceptance, and values with the subscript letter $R$, represent a test case where the expected decision is a rejection.

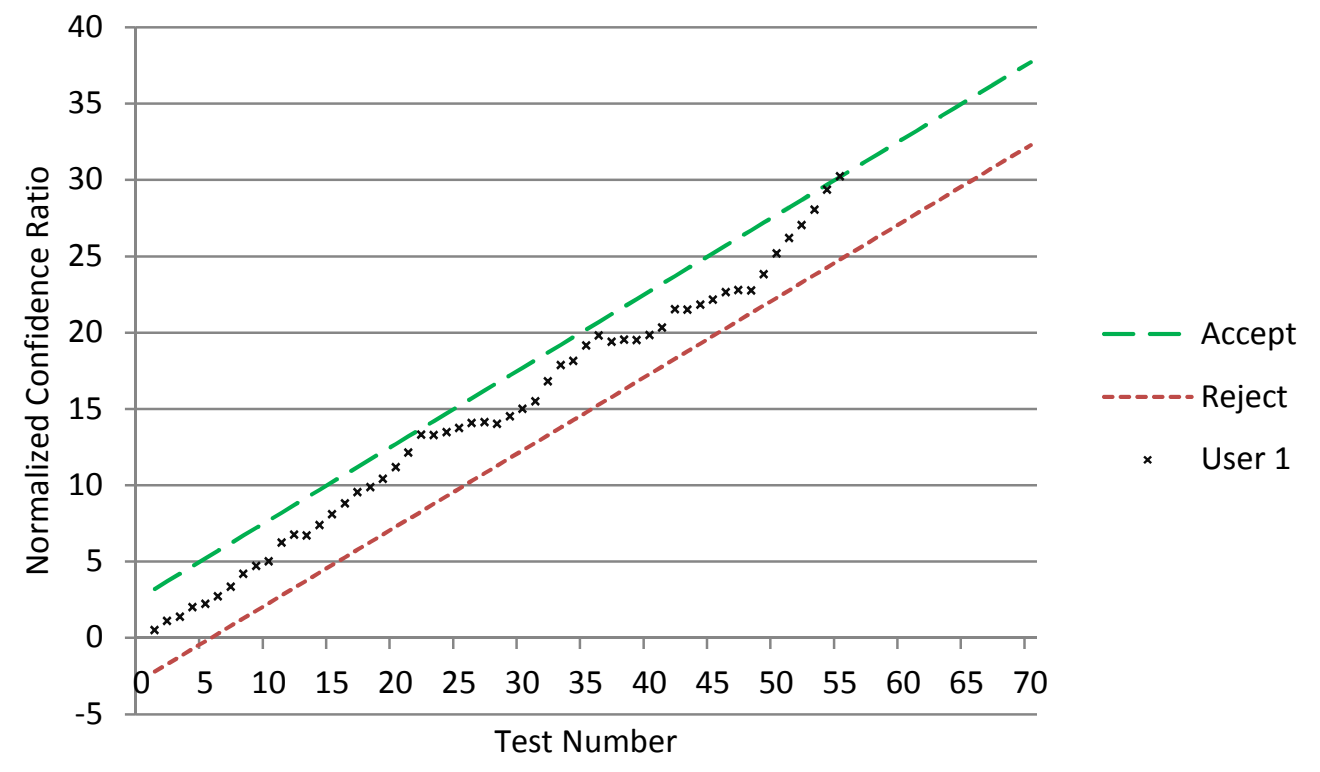

Figure 7: User 1's new input tested on User 1's profile

The user's normalized confidence ratio (CR) navigates through the continue region, until a decision has been made. We can witness a decision being made at test number 55, where the user's normalized confidence ratio crosses the acceptance line and hence the user is accepted. This is an example of a successful trial. Figure 8 demonstrates a test (TID $9 \mathrm{R}$ ) on the same profile, however this time, the profile does not belong to the 
user. By looking at this figure, we can witness that the system comes close to detecting the masquerader near test number 24 , however the confidence ratio climbs as new data is made available. Eventually, this user is rejected at test number 70 , where the confidence ratio penetrates the reject region.

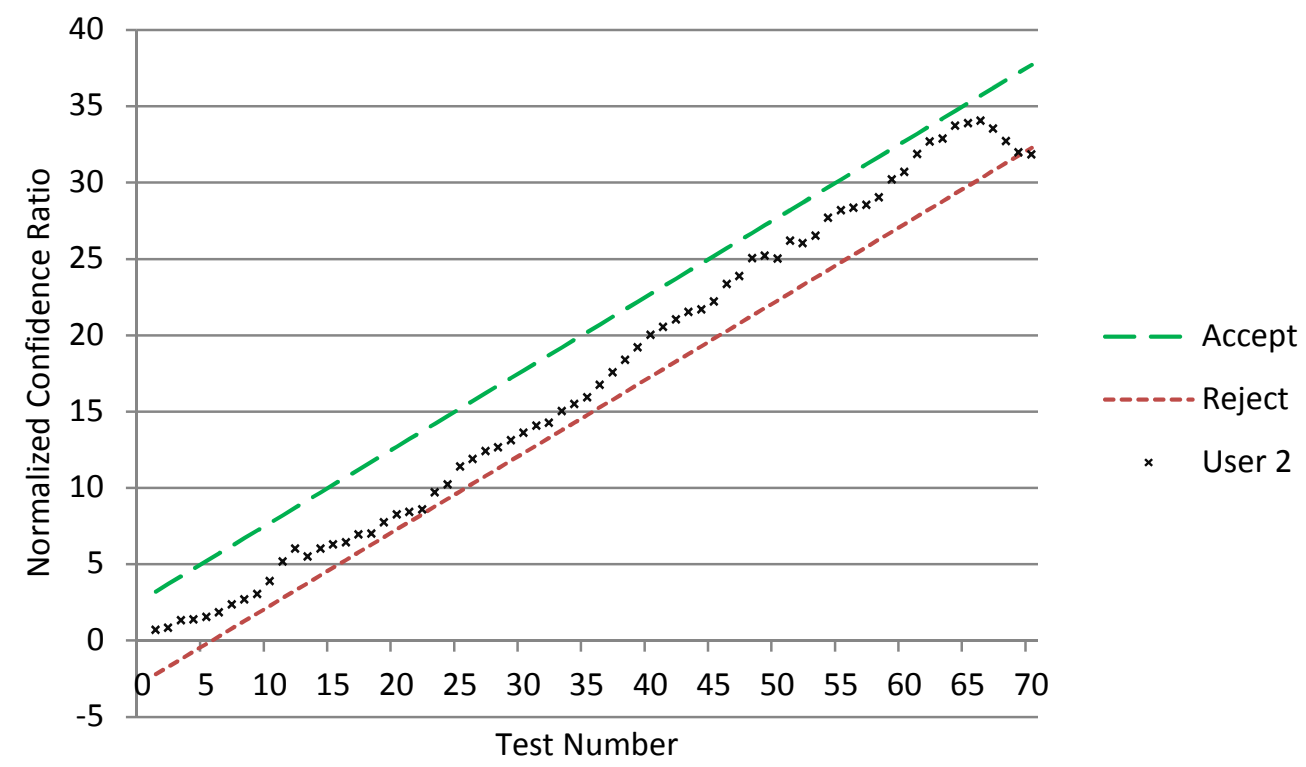

Figure 8: User 2's input tested on User 1's profile

During the course of the system's analysis of the user, we can witness that the decision can go either way. Depending on the sensitivity of the system, decisions can be altered dramatically. For instance, if the continue region was reduced in size, a faster decision would have been made at test number 24 , which is more than $50 \%$ faster than the latter. The challenge lies in determining a sampling plan that will be ideal to the given application. It is important to mention that a sampling plan must be built, such that it compensates for the entire training range.

The following table (Table 26) illustrates the decision making process of the 
system for the previous two examples.

Table 26: Decision making process of the sequential sampling technique

\begin{tabular}{|c|c|c|c|c|c|c|}
\hline $\begin{array}{l}\text { Test } \\
\text { Number }\end{array}$ & $\begin{array}{l}\text { Number of } \\
\text { Commands }\end{array}$ & Accept Limit & Reject Limit & $\begin{array}{c}\text { User 1's } \\
\text { Normalized } \\
\text { CR }\end{array}$ & $\begin{array}{c}\text { User 2's } \\
\text { Normalized } \\
\text { CR }\end{array}$ & Decision \\
\hline 1 & 5 & 3.211632 & -2.21163 & 0.5 & 0.666667 & Continue \\
\hline 2 & 10 & 3.711632 & -1.71163 & 1.111111 & 0.8 & Continue \\
\hline 3 & 15 & 4.211632 & -1.21163 & 1.384615 & 1.285714 & Continue \\
\hline 4 & 20 & 4.711632 & -0.71163 & 2 & 1.333333 & Continue \\
\hline 5 & 25 & 5.211632 & -0.21163 & 2.222222 & 1.5 & Continue \\
\hline 6 & 30 & 5.711632 & 0.288368 & 2.7 & 1.8 & Continue \\
\hline 7 & 35 & 6.211632 & 0.788368 & 3.333333 & 2.333333 & Continue \\
\hline 8 & 40 & 6.711632 & 1.288368 & 4.173913 & 2.666667 & Continue \\
\hline 9 & 45 & 7.211632 & 1.788368 & 4.695652 & 3 & Continue \\
\hline 10 & 50 & 7.711632 & 2.288368 & 5 & 3.846154 & Continue \\
\hline 11 & 55 & 8.211632 & 2.788368 & 6.233333 & 5.133333 & Continue \\
\hline 12 & 60 & 8.711632 & 3.288368 & 6.75 & 6 & Continue \\
\hline 13 & 65 & 9.211632 & 3.788368 & 6.685714 & 5.473684 & Continue \\
\hline 14 & 70 & 9.711632 & 4.288368 & 7.388889 & 6 & Continue \\
\hline 15 & 75 & 10.21163 & 4.788368 & 8.076923 & 6.25 & Continue \\
\hline 16 & 80 & 10.71163 & 5.288368 & 8.8 & 6.4 & Continue \\
\hline 17 & 85 & 11.21163 & 5.788368 & 9.536585 & 6.925926 & Continue \\
\hline 18 & 90 & 11.71163 & 6.288368 & 9.857143 & 6.967742 & Continue \\
\hline 19 & 95 & 12.21163 & 6.788368 & 10.40476 & 7.71875 & Continue \\
\hline 20 & 100 & 12.71163 & 7.288368 & 11.16279 & 8.235294 & Continue \\
\hline 21 & 105 & 13.21163 & 7.788368 & 12.13333 & 8.4 & Continue \\
\hline 22 & 110 & 13.71163 & 8.288368 & 13.29167 & 8.555556 & Continue \\
\hline 23 & 115 & 14.21163 & 8.788368 & 13.26923 & 9.684211 & Continue \\
\hline 24 & 120 & 14.71163 & 9.288368 & 13.47368 & 10.2 & Continue \\
\hline 25 & 125 & 15.21163 & 9.788368 & 13.75 & 11.36364 & Continue \\
\hline 26 & 130 & 15.71163 & 10.28837 & 14.06557 & 11.86957 & Continue \\
\hline 27 & 135 & 16.21163 & 10.78837 & 14.12308 & 12.375 & Continue \\
\hline 28 & 140 & 16.71163 & 11.28837 & 14 & 12.62745 & Continue \\
\hline 29 & 145 & 17.21163 & 11.78837 & 14.5 & 13.07843 & Continue \\
\hline 30 & 150 & 17.71163 & 12.28837 & 15 & 13.58491 & Continue \\
\hline 31 & 155 & 18.21163 & 12.78837 & 15.5 & 14.03774 & Continue \\
\hline 32 & 160 & 18.71163 & 13.28837 & 16.8 & 14.22222 & Continue \\
\hline 33 & 165 & 19.21163 & 13.78837 & 17.85882 & 15 & Continue \\
\hline 34 & 170 & 19.71163 & 14.28837 & 18.13333 & 15.45455 & Continue \\
\hline 35 & 175 & 20.21163 & 14.78837 & 19.15789 & 15.90909 & Continue \\
\hline 36 & 180 & 20.71163 & 15.28837 & 19.8 & 16.71429 & Continue \\
\hline 37 & 185 & 21.21163 & 15.78837 & 19.38095 & 17.52632 & Continue \\
\hline 38 & 190 & 21.71163 & 16.28837 & 19.53271 & 18.34483 & Continue \\
\hline
\end{tabular}




\begin{tabular}{|c|c|c|c|c|c|c|}
\hline 39 & 195 & 22.21163 & 16.78837 & 19.5 & 19.16949 & Continue \\
\hline 40 & 200 & 22.71163 & 17.28837 & 19.82301 & 20 & Continue \\
\hline 41 & 205 & 23.21163 & 17.78837 & 20.32479 & 20.5 & Continue \\
\hline 42 & 210 & 23.71163 & 18.28837 & 21.52066 & 21 & Continue \\
\hline 43 & 215 & 24.21163 & 18.78837 & 21.5 & 21.5 & Continue \\
\hline 44 & 220 & 24.71163 & 19.28837 & 21.824 & 21.66154 & Continue \\
\hline 45 & 225 & 25.21163 & 19.78837 & 22.14286 & 22.16418 & Continue \\
\hline 46 & 230 & 25.71163 & 20.28837 & 22.64063 & 23.33333 & Continue \\
\hline 47 & 235 & 26.21163 & 20.78837 & 22.78788 & 23.84058 & Continue \\
\hline 48 & 240 & 26.71163 & 21.28837 & 22.75556 & 25.01408 & Continue \\
\hline 49 & 245 & 27.21163 & 21.78837 & 23.8 & 25.18056 & Continue \\
\hline 50 & 250 & 27.71163 & 22.28837 & 25.17241 & 25 & Continue \\
\hline 51 & 255 & 28.21163 & 22.78837 & 26.18 & 26.17105 & Continue \\
\hline 52 & 260 & 28.71163 & 23.28837 & 27.02632 & 26 & Continue \\
\hline 53 & 265 & 29.21163 & 23.78837 & 28.03871 & 26.5 & Continue \\
\hline 54 & 270 & 29.71163 & 24.28837 & 29.3625 & 27.65854 & Continue \\
\hline 55 & 275 & 30.21163 & 24.78837 & 30.21605 & 28.15476 & $\begin{array}{l}\text { Accept } \\
\text { User } 1\end{array}$ \\
\hline 56 & 280 & 30.71163 & 25.28837 & & 28.32941 & Continue \\
\hline 57 & 285 & 31.21163 & 25.78837 & & 28.5 & Continue \\
\hline 58 & 290 & 31.71163 & 26.28837 & & 29 & Continue \\
\hline 59 & 295 & 32.21163 & 26.78837 & & 30.17045 & Continue \\
\hline 60 & 300 & 32.71163 & 27.28837 & & 30.66667 & Continue \\
\hline 61 & 305 & 33.21163 & 27.78837 & & 31.82609 & Continue \\
\hline 62 & 310 & 33.71163 & 28.28837 & & 32.66667 & Continue \\
\hline 63 & 315 & 34.21163 & 28.78837 & & 32.84043 & Continue \\
\hline 64 & 320 & 34.71163 & 29.28837 & & 33.68421 & Continue \\
\hline 65 & 325 & 35.21163 & 29.78837 & & 33.85417 & Continue \\
\hline 66 & 330 & 35.71163 & 30.28837 & & 34.02062 & Continue \\
\hline 67 & 335 & 36.21163 & 30.78837 & & 33.5 & Continue \\
\hline 68 & 340 & 36.71163 & 31.28837 & & 32.69231 & Continue \\
\hline 69 & 345 & 37.21163 & 31.78837 & & 31.94444 & Continue \\
\hline 70 & 350 & 37.71163 & 32.28837 & & 31.81818 & $\begin{array}{l}\text { Reject } \\
\text { User } 2 \\
\end{array}$ \\
\hline
\end{tabular}

The two types of tests that were conducted in this experiment include the testing for rejection and the testing for acceptance. Acceptance tests involved the testing of a user against its own profile. The purpose of this test is to calculate the FRR of our intrusion detection system. Given that there are 75 victims, this type of testing did not require vast amount of computational time. Depending on the sample size, the acceptance tests 
did not require more than an hour to complete a single trial on our workstation. However, in the case of a rejection test, 31 masqueraders are used as input to 75 profiles. Depending on the sample size, this type of test can take up to 10 hours to complete a single trial on our workstation. The purpose of a rejection test is to calculate the FAR of our intrusion detection system.

The following table (Table 27) illustrates the final results that we have achieved by implementing a Naïve Bayes learning mechanism in conjunction with the decision making of the sequential sampling technique.

Table 27: Results achieved based on different parameters and sampling sizes

\begin{tabular}{|l|l|l|l|l|l|l|l|l|l|l|l|l|}
\hline $\begin{array}{c}\text { Test } \\
\text { ID }\end{array}$ & $\begin{array}{c}\text { Sample } \\
\text { Size } \\
\text { N } \\
\text { (Actions) }\end{array}$ & $p_{1}$ & $p_{2}$ & $\boldsymbol{\alpha}$ & $\boldsymbol{\beta}$ & $\begin{array}{c}\text { FAR } \\
(\%)\end{array}$ & $\begin{array}{c}\text { FRR } \\
(\%)\end{array}$ & $\begin{array}{c}\text { DR } \\
(\%)\end{array}$ & $\begin{array}{c}\text { Min } \\
\text { TTA } \\
\text { (Commands) }\end{array}$ & $\begin{array}{c}\text { Max } \\
\text { TAA } \\
\text { (cctions) }\end{array}$ & $\begin{array}{c}\text { Mean } \\
\text { TTA } \\
\text { (Actions) }\end{array}$ & $\begin{array}{c}\text { CPU } \\
\text { Usage } \\
\text { (Seconds) }\end{array}$ \\
\hline & 3 & 0.27 & 0.73 & 0.01 & 0.01 & 03.225 & & 96.78 & 12 & 183 & 16.28 & \\
\hline & 3 & 0.28 & 0.72 & 0.01 & 0.01 & 03.183 & & 96.82 & 12 & 186 & 16.61 & \\
\hline $\mathbf{1}$ & 3 & 0.29 & 0.71 & 0.01 & 0.01 & 02.968 & 12.00 & 97.03 & 15 & 198 & 19.35 & 6.97 \\
\hline $\mathbf{2}$ & 3 & 0.30 & 0.70 & 0.01 & 0.01 & 02.882 & 12.00 & 97.12 & 15 & 237 & 20.09 & 7.20 \\
\hline $\mathbf{3}$ & 3 & 0.31 & 0.69 & 0.01 & 0.01 & 02.882 & 12.00 & 97.12 & 15 & 252 & 20.60 & 7.35 \\
\hline $\mathbf{4}$ & 3 & 0.32 & 0.68 & 0.01 & 0.01 & 02.796 & 12.00 & 97.20 & 18 & 252 & 23.63 & - \\
\hline $\mathbf{5}$ & 3 & 0.33 & 0.67 & 0.01 & 0.01 & 02.581 & 12.00 & 97.42 & 18 & 300 & 24.46 & - \\
\hline $\mathbf{6}$ & 3 & 0.34 & 0.66 & 0.01 & 0.01 & 02.581 & 12.00 & 97.42 & 18 & 546 & 25.25 & - \\
\hline $\mathbf{7}$ & 3 & 0.35 & 0.65 & 0.01 & 0.01 & 02.581 & 12.00 & 97.42 & 21 & 552 & 29.13 & - \\
\hline $\mathbf{8}$ & 5 & 0.29 & 0.71 & 0.01 & 0.01 & 02.237 & 12.00 & 97.76 & 25 & 555 & 34.26 & 10.42 \\
\hline $\mathbf{9}$ & 5 & 0.30 & 0.70 & 0.01 & 0.01 & 02.237 & 12.00 & 97.76 & 25 & 575 & 35.17 & 10.47 \\
\hline $\mathbf{1 0}$ & 5 & 0.31 & 0.69 & 0.01 & 0.01 & 02.237 & 12.00 & 97.76 & 25 & 575 & 36.16 & 10.59 \\
\hline $\mathbf{1 1}$ & 10 & 0.29 & 0.71 & 0.01 & 0.01 & 01.464 & 13.33 & 98.41 & 50 & 910 & 67.94 & 17.61 \\
\hline $\mathbf{1 2}$ & 10 & 0.30 & 0.70 & 0.01 & 0.01 & 01.421 & 13.33 & 98.45 & 50 & 910 & 70.22 & 18.10 \\
\hline $\mathbf{1 3}$ & 10 & 0.31 & 0.69 & 0.01 & 0.01 & 01.421 & 13.33 & 98.41 & 50 & 920 & 72.59 & 18.75 \\
\hline $\mathbf{1 4}$ & 15 & 0.29 & 0.71 & 0.01 & 0.01 & - & 12.00 & & - & - & - & - \\
\hline $\mathbf{1 5}$ & 15 & 0.30 & 0.70 & 0.01 & 0.01 & - & 12.00 & & - & - & - & - \\
\hline $\mathbf{1 6}$ & 15 & 0.31 & 0.69 & 0.01 & 0.01 & - & 12.00 & & - & - & - & - \\
\hline $\mathbf{1 7}$ & 20 & 0.30 & 0.70 & 0.01 & 0.01 & - & 13.33 & & - & - & - & - \\
\hline $\mathbf{1 8}$ & 20 & 0.31 & 0.69 & 0.01 & 0.01 & - & 13.33 & & - & - & - & - \\
\hline $\mathbf{1 9}$ & 20 & 0.29 & 0.71 & 0.01 & 0.01 & - & 13.33 & & - & - & - & - \\
\hline
\end{tabular}

Looking at the results, a few patterns are visible. We can witness that as the sample size increases the FAR rate is decreased. A larger sample size allows the system to gain a better understanding of the given user, before making a decision. Figure 9 illustrates 
the three different sampling plans used in testing the system. We can clearly notice that as the sample size is increased, the FAR rate decreases.

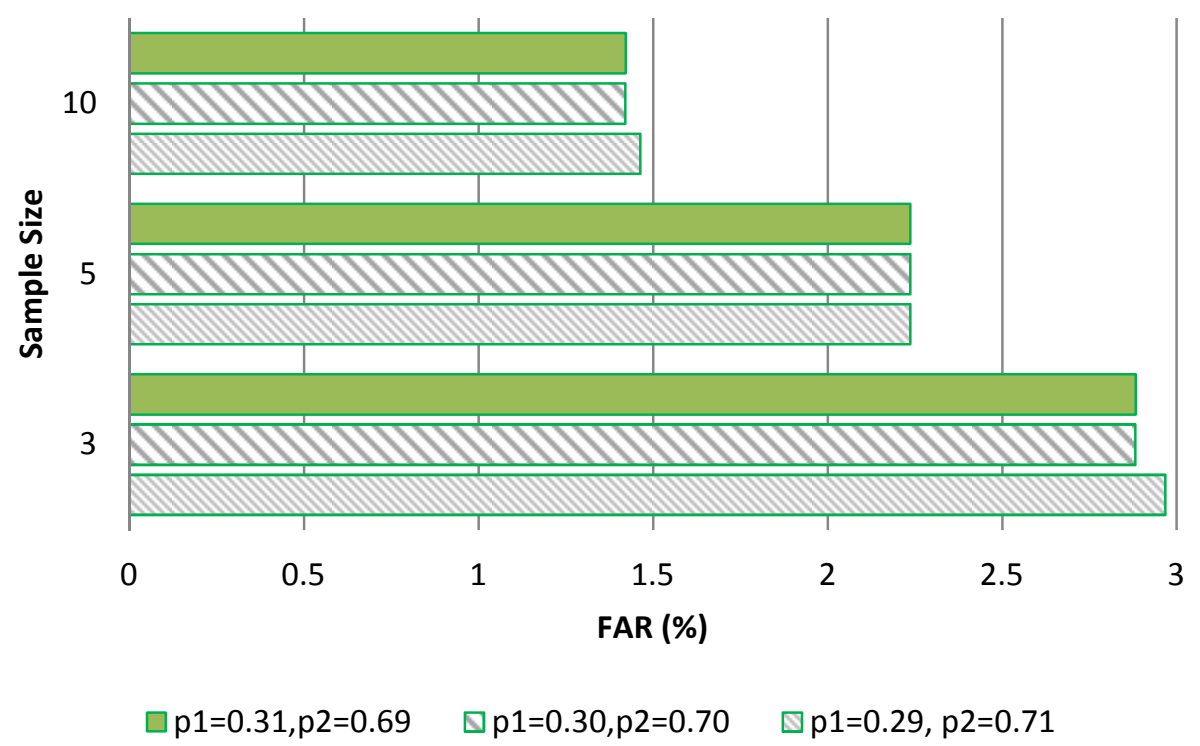

Figure 9: Analysing the system's FAR rate versus the sample size

Figure 10 illustrates the analysis of the FRR rate as the sample size increases. We can witness that as the sample size increases the FRR rate also increased. 


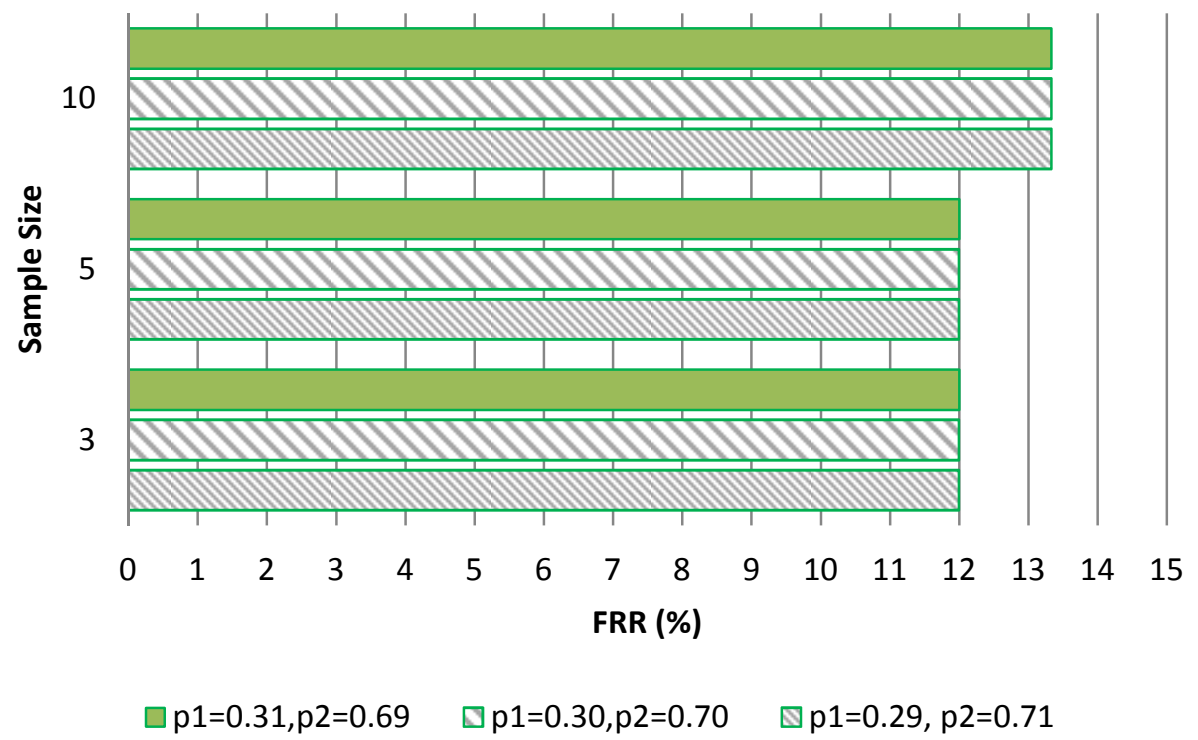

Figure 10: Analysing the system's FRR rate versus the sample size

Figure 11 illustrates the pattern of the MTTA value as the sample size is increased. It is observed that the Time-to-Alarm (TTA) is increased as the sample size is increased.

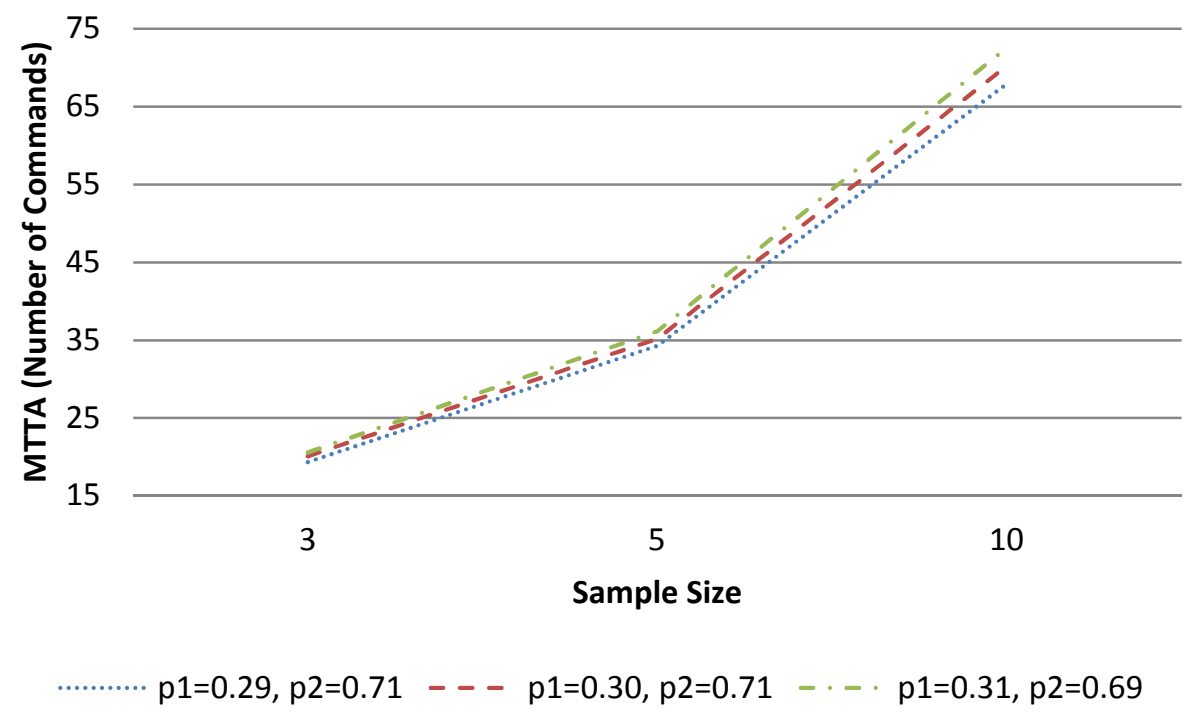

Figure 11: Analysis of the system's MTTA value as the sample size is increased

Figure 12 shows a closer view at the effects of the sample size on the MTTA value. We 
can observed that depending on the sampling plan, the MTTA is also affected. This is understandable due to the fact that as the lower threshold is increased within the sampling plan, the continue region is also increased in size. Therefore, the time spent in the continue region is increased.

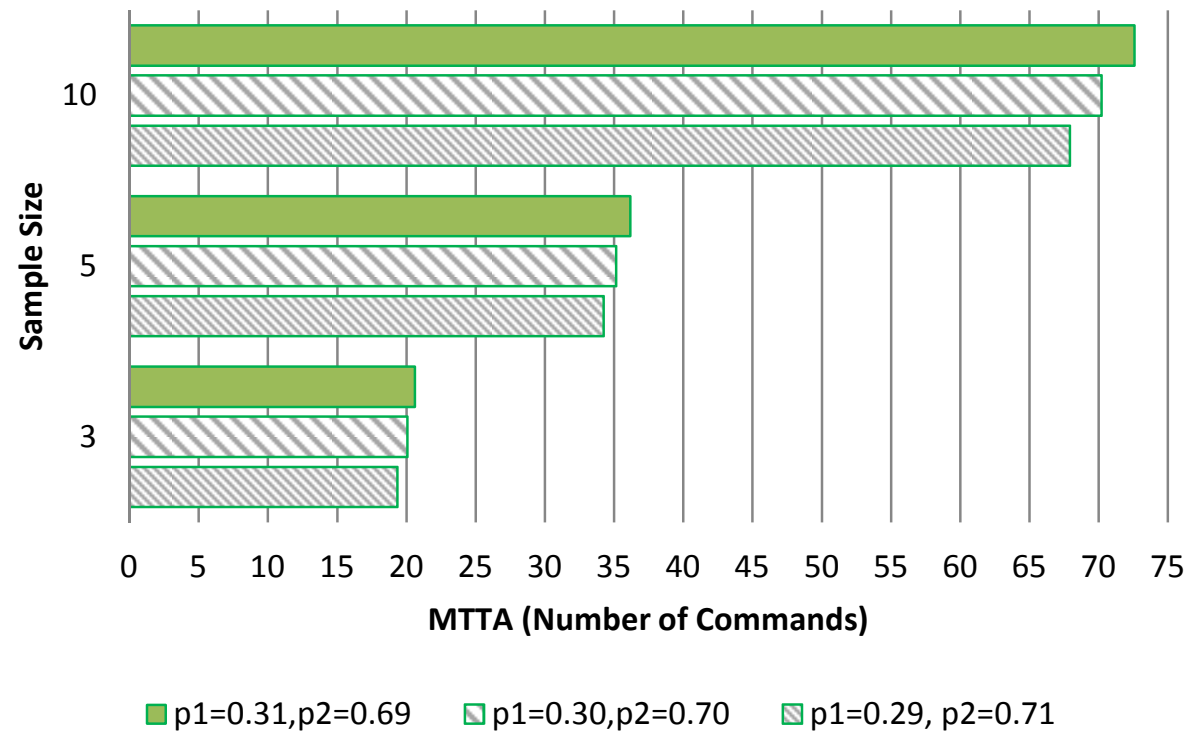

Figure 12: Analysing the system's MTTA value versus the sample size

Figure 13 illustrates the pattern between the CPU usages and the different sample sizes. Naturally, the CPU usage follows the same pattern as the MTTA value. Depending on the workstation used, the values in seconds are different. However, the illustrated pattern should remain the same. 


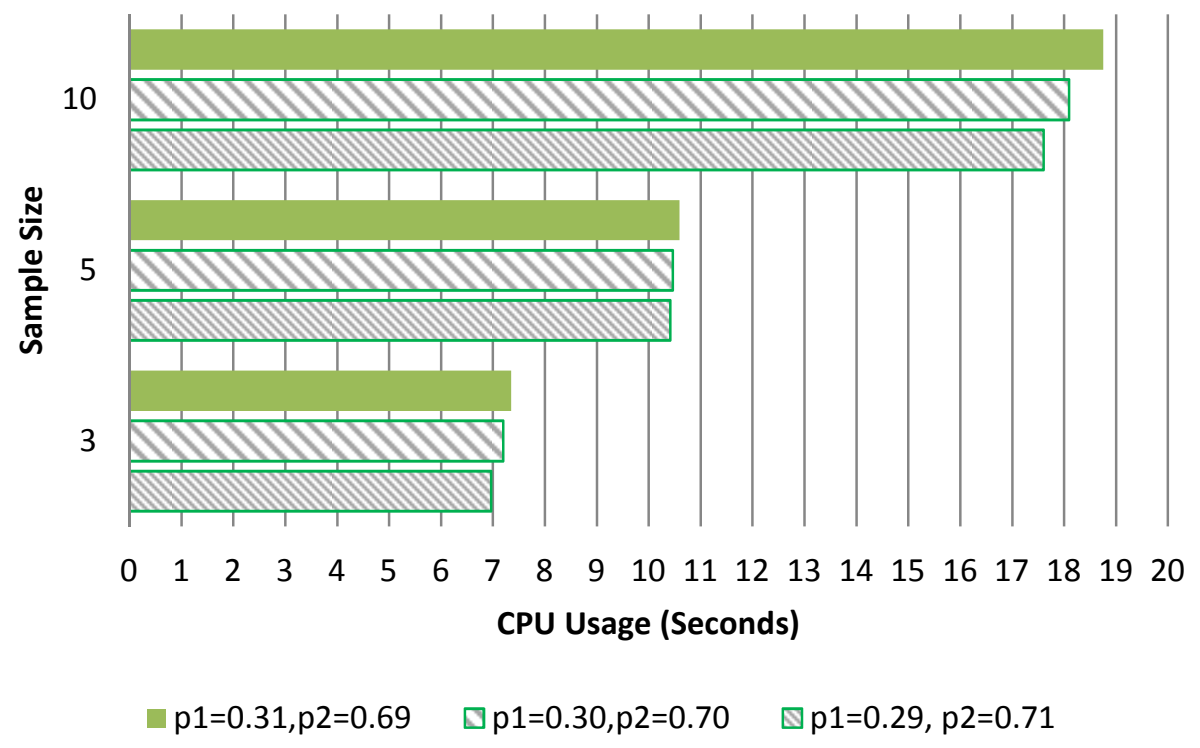

Figure 13: Analyzing the systems CPU Usage versus the sample size

A brief discussion on the given results will be given in the next section.

\subsection{Discussion}

As stated earlier, it is the goal of any intrusion detection system to reduce the FAR, FRR and the MTTA values. The challenge lies in finding suitable approaches that can accomplish this task in an efficient way. Looking at our results, we can witness a common trend, the more accurate our results are, the more time is consumed. Figure 14, Figure 15 and Figure 16 show the relationship between the FRR and the FAR value in three different sampling plans, using three different sample sizes. 


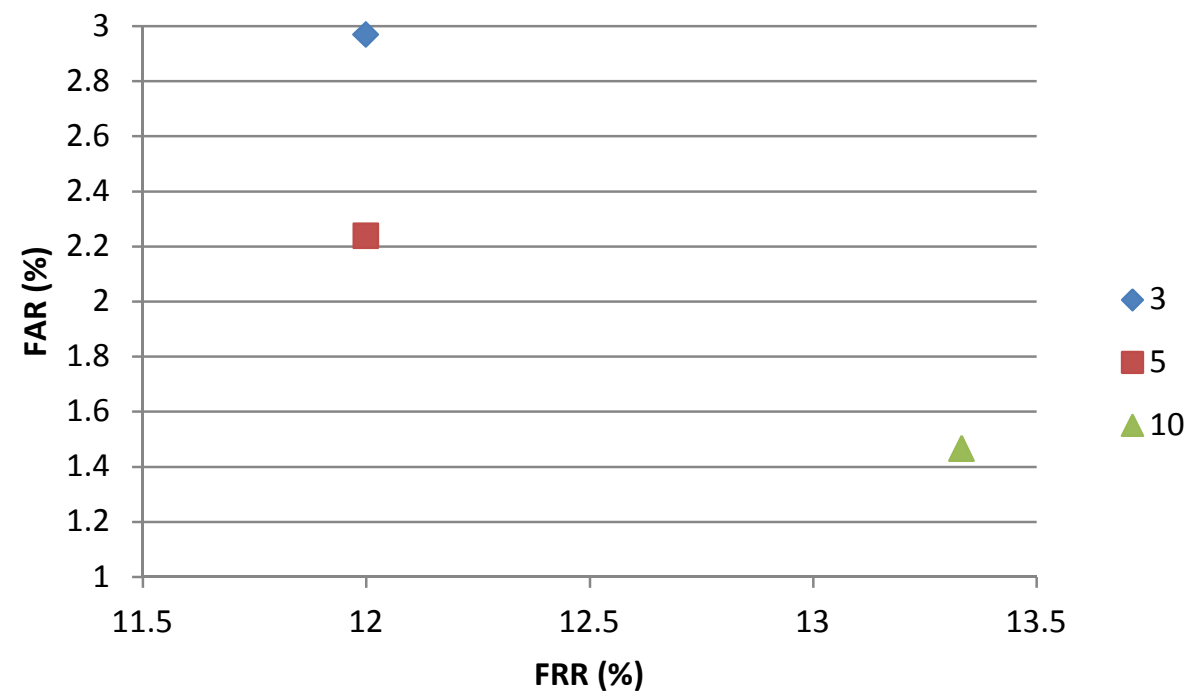

Figure 14: Relationship between sample size and FRR/FAR using sampling plan $A$

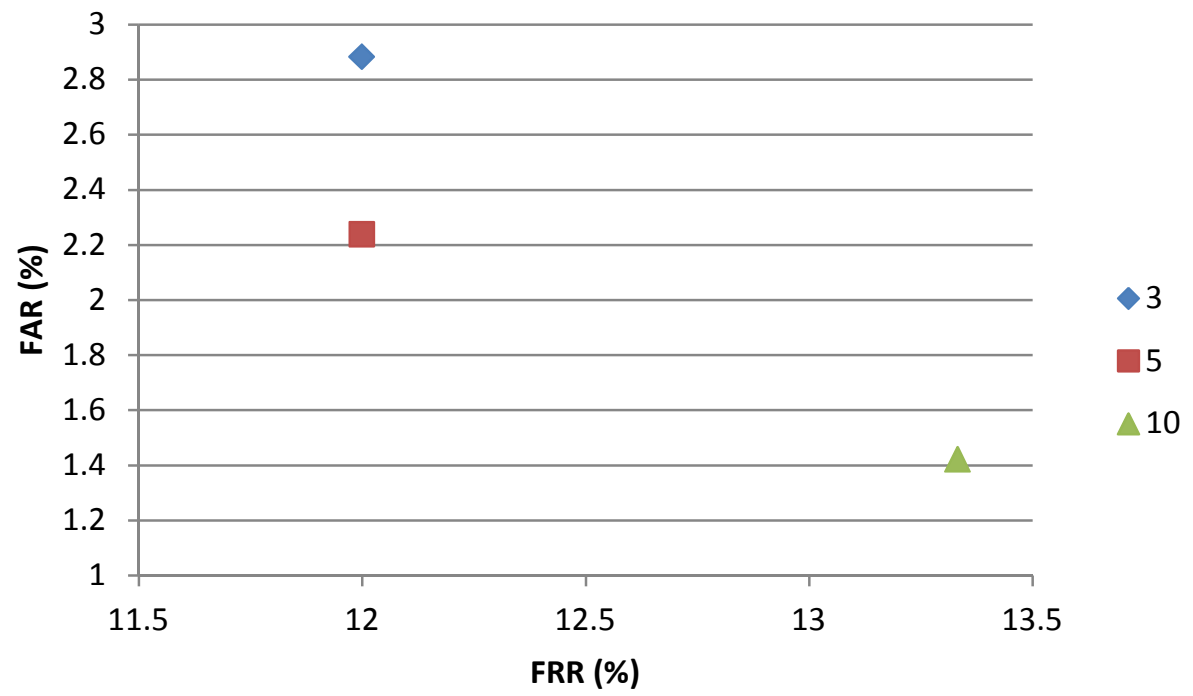

Figure 15: Relationship between sample size and FRR/FAR using sampling plan B 


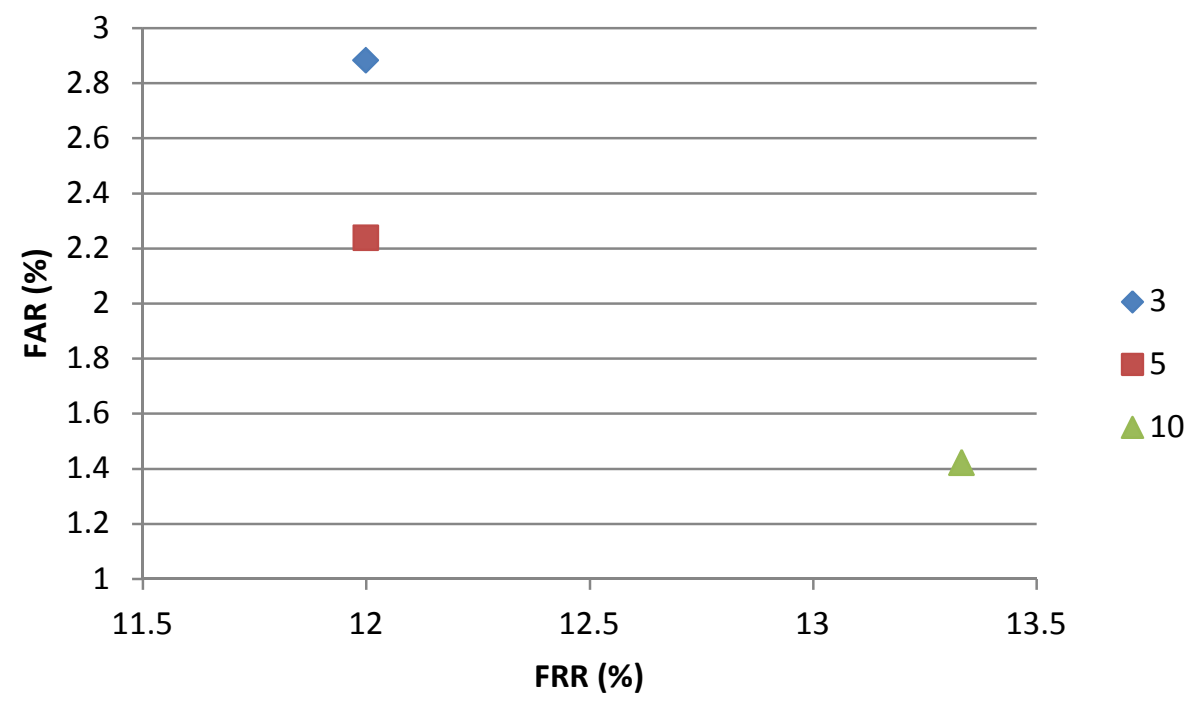

Figure 16: Relationship between sample size and FRR/FAR using sampling plan $C$ It is important to acknowledge that the data used to calculate the FRR rate as opposed to the FAR rate was minimal. In order to calculate the FRR rate, 75 cases were tested, whereas the FAR rate was calculated by testing 3,235 cases. The FRR rate may become quite different if a more extensive testing procedure is applied.

The results show a promising range of detection rates. Depending on the sampling plan and sample size, the detection rate ranged from $96.78 \%$ to $98.41 \%$. These values represent a promising system compared to other proposed intrusion detection systems [15]. Although there are not many literatures in the intrusion detection field where the Greenberg dataset has been used used, comparing these results solely on the basis of the outcome achieved, we can confirm that the results produced are quite competitive.

Depending on the ranking function used to evaluate the integrity of the system, the ranking results can vary. Hence, the emphasis can be set either on type I errors or 
type II errors. The following ranking function [10] represents the foundation for determining such emphasis.

$$
\text { Cost }=\alpha(F A R)+\beta(F R R)
$$

If there is no preference to the type of error considered, the $\alpha$ and $\beta$ attributes can be ignored, i.e. set to 1 . Therefore, in order to calculate the cost of a given detection algorithm, we add the FAR and FRR rates. Table 28 shows comparison comparative study of numerous detection methods based on a ranking function that does not emphasize on a particular type of error.

Table 28: Result comparison based on Cost $=(F A R)+(F R R)$

\begin{tabular}{|l|c|c|c|c|c|c|l|}
\hline Method & Cost & $\begin{array}{c}\text { DR } \\
(\%)\end{array}$ & $\begin{array}{c}\text { FAR } \\
(\%)\end{array}$ & $\begin{array}{c}\text { FRR } \\
(\%)\end{array}$ & $\begin{array}{c}\text { Sample } \\
\text { Size }\end{array}$ & Trained & Data \\
\hline N. Bayes Classifier & 33.8 & 70.9 & 29.1 & 4.7 & 10 & 1000 & Greenberg \\
\hline N. Bayes Classifier & 23.6 & 82.1 & 17.9 & 5.7 & 10 & 1000 & Greenberg \\
\hline CABLUCS & 14.97 & 97.3 & 2.97 & 12 & 3 & 1000 & Greenberg \\
\hline CABLUCS & 14.24 & 97.76 & 2.24 & 12 & 5 & 1000 & Greenberg \\
\hline CABLUCS & 14.75 & 98.45 & 1.42 & 13.33 & 10 & 1000 & Greenberg \\
\hline $\begin{array}{l}\text { Customized } \\
\text { Grammars }\end{array}$ & 14.1 & 93.1 & 6.9 & 7.2 & - & - & SEA \\
\hline $\begin{array}{l}\text { Customized } \\
\text { Grammars }\end{array}$ & 30.6 & 71.0 & 29.0 & 1.6 & - & - & SEA \\
\hline $\begin{array}{l}\text { Self Signature with } \\
\text { Uniqueness }\end{array}$ & 14.6 & 91.3 & 8.7 & 5.9 & - & - & SEA \\
\hline $\begin{array}{l}\text { Self Signature with } \\
\text { Uniqueness }\end{array}$ & 33.9 & 67.5 & 32.5 & 1.4 & - & - & SEA \\
\hline $\begin{array}{l}\text { Boosting Decision } \\
\text { Stumps }\end{array}$ & 20.9 & 89.2 & 10.8 & 10.1 & - & - & SEA \\
\hline SVM & 29.6 & 80.1 & 19.9 & 9.7 & - & - & SEA \\
\hline ECM & 30.2 & 72.3 & 27.7 & 2.5 & - & - & SEA \\
\hline $\begin{array}{l}\text { N. Bayes (no } \\
\text { updating) }\end{array}$ & 38.4 & 66.2 & 33.8 & 4.6 & - & - & SEA \\
\hline N. Bayes (updating) & 39.8 & 61.5 & 38.5 & 1.3 & - & - & SEA \\
\hline Uniqueness & 62 & 39.4 & 60.6 & 1.4 & - & - & SEA \\
\hline IPAM & 61.3 & 41.4 & 58.6 & 2.7 & - & - & SEA \\
\hline Hybrid Markov & 53.9 & 49.3 & 50.7 & 3.2 & - & - & SEA \\
\hline
\end{tabular}




\begin{tabular}{|l|c|c|c|c|c|c|l|}
\hline Sequence match & 66.9 & 36.8 & 63.2 & 3.7 & - & - & SEA \\
\hline Compression & 70.8 & 34.2 & 65.8 & 5.0 & - & - & SEA \\
\hline $\begin{array}{l}\text { Bayes one-step } \\
\text { Markov }\end{array}$ & 37.4 & 69.3 & 30.7 & 6.7 & - & - & SEA \\
\hline
\end{tabular}

Using this ranking function, we can witness that our system ranks $1^{\text {st }}$ in the detection methods that use the Greenberg dataset, and ranks $2^{\text {nd }}$ overall, regardless of the dataset used.

Table 29: Result ranking comparison based on Cost $=(F A R)+(F R R)$

\begin{tabular}{|l|l|l|l|}
\hline Rank & Method & Cost & Dataset \\
\hline 1 & Customized Grammars & 14.1 & SEA \\
\hline 2 & CABLUCS & 14.24 & Greenberg \\
\hline 3 & Self Signature with Uniqueness & 14.6 & SEA \\
\hline 4 & CABLUCS & 14.75 & Greenberg \\
\hline 5 & CABLUCS S & 14.97 & Greenberg \\
\hline 6 & Boosting Decision Stumps & 20.9 & SEA \\
\hline 7 & Naïve Bayes Classifier & 23.6 & Greenberg \\
\hline 8 & SVM & 29.6 & SEA \\
\hline 9 & ECM & 30.2 & SEA \\
\hline 10 & Customized Grammars & 30.6 & SEA \\
\hline 11 & Naïve Bayes Classifier & 33.8 & Greenberg \\
\hline 12 & Self Signature with Uniqueness & 33.9 & SEA \\
\hline 13 & Bayes one-step Markov & 37.4 & SEA \\
\hline 14 & Naïve Bayes (no updating) & 38.4 & SEA \\
\hline 15 & Naïve Bayes (updating) & 39.8 & SEA \\
\hline 16 & Hybrid Markov & 53.9 & SEA \\
\hline 17 & IPAM & 61.3 & SEA \\
\hline 18 & Uniqueness & 62 & SEA \\
\hline 19 & Sequence match & 66.9 & SEA \\
\hline 20 & Compression & 70.8 & SEA \\
\hline & & & \\
\hline
\end{tabular}

The SEA dataset is the work of Schonlau et al. [9], which is a more commonly used benchmark dataset. In their work, the emphasis was set based on achieving a 1\% FRR rate [10]. After the completion of their tests, the only successful method to achieve the given FRR rate was Uniqueness. In order to rank Uniqueness as the best detection 
method based on the given criteria, the $\beta$ (type II error) emphasis was set to 6 . Table 30 shows the results in comparison to the Schonlau et al. ranking function.

Table 30: Result comparison based on Cost $=(F A R)+6(F R R)$

\begin{tabular}{|l|c|c|c|c|c|c|l|}
\hline Method & Cost & $\begin{array}{c}\text { DR } \\
(\%)\end{array}$ & $\begin{array}{c}\text { FAR } \\
(\%)\end{array}$ & $\begin{array}{c}\text { FRR } \\
(\%)\end{array}$ & $\begin{array}{c}\text { Sample } \\
\text { Size }\end{array}$ & Trained & Dataset \\
\hline N. Bayes Classifier & 57.3 & 70.9 & 29.1 & 4.7 & 10 & 1000 & Greenberg \\
\hline $\begin{array}{l}\text { Naïve Bayes } \\
\text { Classifier }\end{array}$ & 52.1 & 82.1 & 17.9 & 5.7 & 10 & 1000 & Greenberg \\
\hline CABLUCS & 74.97 & 97.3 & 2.97 & 12 & 3 & 1000 & Greenberg \\
\hline CABLUCS & 74.24 & 97.76 & 2.24 & 12 & 5 & 1000 & Greenberg \\
\hline CABLUCS & 81.4 & 98.45 & 1.42 & 13.33 & 10 & 1000 & Greenberg \\
\hline $\begin{array}{l}\text { Customized } \\
\text { Grammars }\end{array}$ & 14.1 & 93.1 & 6.9 & 7.2 & - & - & SEA \\
\hline $\begin{array}{l}\text { Customized } \\
\text { Grammars }\end{array}$ & 30.6 & 71.0 & 29.0 & 1.6 & - & - & SEA \\
\hline $\begin{array}{l}\text { Self Signature with } \\
\text { Uniqueness }\end{array}$ & 14.6 & 91.3 & 8.7 & 5.9 & - & - & SEA \\
\hline $\begin{array}{l}\text { Self Signature with } \\
\text { Uniqueness }\end{array}$ & 33.9 & 67.5 & 32.5 & 1.4 & - & - & SEA \\
\hline $\begin{array}{l}\text { Boosting Decision } \\
\text { Stumps }\end{array}$ & 20.9 & 89.2 & 10.8 & 10.1 & - & - & SEA \\
\hline SVM & 29.6 & 80.1 & 19.9 & 9.7 & - & - & SEA \\
\hline ECM & 30.2 & 72.3 & 27.7 & 2.5 & - & - & SEA \\
\hline $\begin{array}{l}\text { N. Bayes (no } \\
\text { updating) }\end{array}$ & 38.4 & 66.2 & 33.8 & 4.6 & - & - & SEA \\
\hline N. Bayes (updating) & 39.8 & 61.5 & 38.5 & 1.3 & - & - & SEA \\
\hline Uniqueness & 62.0 & 39.4 & 60.6 & 1.4 & - & - & SEA \\
\hline IPAM & 61.3 & 41.4 & 58.6 & 2.7 & - & - & SEA \\
\hline Hybrid Markov & 53.9 & 49.3 & 50.7 & 3.2 & - & - & SEA \\
\hline Sequence match & 66.9 & 36.8 & 63.2 & 3.7 & - & - & SEA \\
\hline Compression & 70.8 & 34.2 & 65.8 & 5.0 & - & - & SEA \\
\hline $\begin{array}{l}\text { Bayes one-step } \\
\text { Markov }\end{array}$ & 37.4 & 69.3 & 30.7 & 6.7 & - & - & SEA \\
\hline
\end{tabular}

Table 31 shows the ranking comparison of the detection methods based on the new criteria. We can witness that the results have dramatically changed given that the emphasis is now based on the type II error. Considering the relatively high FRR rate of 
our system in comparison to other mentioned detection methods, it comes as no surprise that our system is now ranked the lowest.

Table 31: Result ranking comparison based on Cost $=(F A R)+6(F R R)$

\begin{tabular}{|l|l|l|l|}
\hline Rank & Method & Cost & Dataset \\
\hline 1 & Customized Grammars & 14.1 & SEA \\
\hline 2 & Self Signature with Uniqueness & 14.6 & SEA \\
\hline 3 & Boosting Decision Stumps & 20.9 & SEA \\
\hline 4 & SVM & 29.6 & SEA \\
\hline 5 & ECM & 30.2 & SEA \\
\hline 6 & Customized Grammars & 30.6 & SEA \\
\hline 7 & Self Signature with Uniqueness & 33.9 & SEA \\
\hline 8 & Bayes one-step Markov & 37.4 & SEA \\
\hline 9 & N. Bayes (no updating) & 38.4 & SEA \\
\hline 10 & N. Bayes (updating) & 39.8 & SEA \\
\hline 11 & Naïve Bayes Classifier & 52.1 & Greenberg \\
\hline 12 & Hybrid Markov & 53.9 & SEA \\
\hline 13 & N. Bayes Classifier & 57.3 & Greenberg \\
\hline 14 & IPAM & 61.3 & SEA \\
\hline 15 & Uniqueness & 62 & SEA \\
\hline 16 & Sequence match & 66.9 & SEA \\
\hline 17 & Compression & 70.8 & SEA \\
\hline 18 & CABLUCS & 74.24 & Greenberg \\
\hline 19 & CABLUCS & 74.97 & Greenberg \\
\hline 20 & CABLUCS & 81.4 & Greenberg \\
\hline
\end{tabular}

Depending on the favouritism of the ranking function, each detection method can be ranked and used differently, in contingent with the application in question. It can be said that the FAR value of our system shows a more accurate representation of our detection method than the FRR rate. As previously stated, the FAR value is determined after numerous testing for each given trial (3,235 cases per trial), while the FRR value is tested using only 75 cases per trial. Hence, a single incident of a false rejection can significantly skew the overall results. 
Looking at the cases where the false rejections were witnessed, we can gain a better understanding for the reasoning behind our high FRR rate. Table 32 shows the 13 profiles that were consistently rejected throughout 21 different test cases. Our high FRR rate is based on $17.33 \%$ of our victims that have an average probability of $72.16 \%$ in producing a false rejection. Further investigation of the relative sampling plans and the sequential progression in the decision making process of these 13 profiles can demonstrate the reasoning for such consistently high rejection rates.

Table 32 :Users that were falsely rejected in 21 different test cases

\begin{tabular}{|l|l|}
\hline Uid & FRR (\%) \\
\hline $\mathbf{1 0}$ & 100 \\
\hline $\mathbf{1 3}$ & 4.76 \\
\hline $\mathbf{1 9}$ & 100 \\
\hline $\mathbf{2 0}$ & 33.33 \\
\hline $\mathbf{3 0}$ & 76.19 \\
\hline $\mathbf{4 0}$ & 100 \\
\hline $\mathbf{4 3}$ & 100 \\
\hline $\mathbf{4 5}$ & 100 \\
\hline $\mathbf{7 1}$ & 52.38 \\
\hline $\mathbf{1 2 6}$ & 14.29 \\
\hline $\mathbf{1 2 8}$ & 33.33 \\
\hline $\mathbf{1 2 9}$ & 76.19 \\
\hline $\mathbf{1 5 4}$ & 47.62 \\
\hline $\mathbf{1 5 7}$ & 100 \\
\hline
\end{tabular}




\section{Chapter 5: Conclusion}

In this thesis, we have proposed a hybrid approach based on learning user command sequence for detecting classical masquerade attacks. Our approach (so-called CABLUCS) consisted of two methods, the Naïve Bayes classifier and the sequential sampling technique, used to enhance the capability of a continuous authentication mechanism within an intrusion detection system. In addition, a newly structured dataset was formed using the Greenberg raw dataset, in such a way as to maximize its usability and efficiency. Using this newly structured dataset, a general statistical analysis of the given data was produced, which can be quite helpful to future researchers using the Greenberg dataset. Through experimental evaluation, we found that our scheme achieves a significant improvement over the Maxion and Townsend scheme in terms of accuracy detection.

We believe that this performance is largely attributed to the contribution of the part of our approach that deal with sequential sampling technique for continuous authentication, which constitutes the core of the decision making regarding the legitimacy of a user.

Departing from the results achieved in this thesis, we can infer that our technique can provide significant advancement to the field of masquerade detection, by opening the possibility of exploring the method to other areas of anomaly detection. This can be classified as future work. 


\section{Appendix A: Generated Statistics for the Greenberg Dataset (Ordered by Commands)}

\begin{tabular}{|c|c|c|c|c|c|c|}
\hline User & Uid & Commands & History & Errors & Aliases & Lines \\
\hline scientist-36 & 20 & 12056 & 488 & 566 & 3161 & 73434 \\
\hline scientist-52 & 15 & 7705 & 231 & 299 & 717 & 47280 \\
\hline scientist-42 & 10 & 6068 & 6 & 644 & 3243 & 37598 \\
\hline experienced-7 & 55 & 5857 & 67 & 612 & 2926 & 35896 \\
\hline scientist-18 & 48 & 5584 & 6 & 240 & 1816 & 34258 \\
\hline non-4 & 106 & 5050 & 18 & 161 & 3296 & 30830 \\
\hline scientist -40 & 38 & 4605 & 0 & 98 & 628 & 29020 \\
\hline experienced-20 & 53 & 4556 & 435 & 370 & 1646 & 28054 \\
\hline scientist -4 & 23 & 4507 & 178 & 320 & 789 & 27992 \\
\hline experienced-35 & 78 & 4272 & 28 & 169 & 2504 & 26290 \\
\hline scientist-37 & 32 & 4187 & 121 & 83 & 1866 & 25604 \\
\hline novice-46 & 146 & 4163 & 112 & 372 & 1909 & 26080 \\
\hline scientist -9 & 26 & 4067 & 224 & 65 & 665 & 25424 \\
\hline non-20 & 99 & 4042 & 165 & 124 & 2122 & 24798 \\
\hline experienced -5 & 62 & 4015 & 35 & 222 & 910 & 25220 \\
\hline experienced-28 & 74 & 3893 & 78 & 60 & 2516 & 25116 \\
\hline scientist-27 & 37 & 3817 & 102 & 85 & 0 & 23344 \\
\hline experienced -4 & 75 & 3776 & 2 & 123 & 1329 & 23258 \\
\hline scientist-38 & 24 & 3775 & 48 & 168 & 1312 & 23172 \\
\hline experienced-1 & 80 & 3714 & 174 & 298 & 1906 & 22830 \\
\hline scientist-13 & 42 & 3593 & 357 & 118 & 204 & 21988 \\
\hline scientist-25 & 30 & 3508 & 7 & 122 & 379 & 22706 \\
\hline scientist-14 & 47 & 3433 & 178 & 183 & 2 & 21404 \\
\hline novice-19 & 164 & 3401 & 7 & 363 & 0 & 20816 \\
\hline scientist-23 & 16 & 3360 & 52 & 135 & 481 & 21454 \\
\hline experienced-24 & 86 & 3331 & 222 & 228 & 1456 & 20440 \\
\hline novice -36 & 142 & 3213 & 0 & 137 & 0 & 19840 \\
\hline novice-14 & 126 & 3194 & $O$ & 208 & 0 & 19786 \\
\hline novice-33 & 122 & 3127 & 0 & 106 & 0 & 19556 \\
\hline scientist-43 & 39 & 3106 & 0 & 101 & 546 & 19066 \\
\hline scientist-2 & 25 & 2954 & 236 & 149 & 1656 & 18514 \\
\hline experienced -8 & 68 & 2930 & 67 & 265 & 625 & 18114 \\
\hline scientist-19 & 43 & 2831 & 106 & 112 & 1330 & 17560 \\
\hline experienced-22 & 73 & 2814 & 325 & 122 & 560 & 17478 \\
\hline scientist-20 & 45 & 2697 & 74 & 189 & 804 & 17080 \\
\hline scientist-29 & 17 & 2683 & 20 & 243 & 530 & 16632 \\
\hline scientist-34 & 52 & 2639 & 15 & 88 & 910 & 16648 \\
\hline scientist -46 & 3 & 2551 & 80 & 110 & 495 & 16480 \\
\hline scientist-12 & 28 & 2499 & 53 & 52 & 1162 & 15412 \\
\hline novice-1 & 151 & 2457 & 37 & 213 & 1381 & 14960 \\
\hline novice-12 & 118 & 2436 & 0 & 210 & 0 & 16366 \\
\hline experienced-21 & 59 & 2394 & 157 & 83 & 974 & 14762 \\
\hline experienced -9 & 71 & 2351 & 86 & 136 & 502 & 14500 \\
\hline experienced -17 & 79 & 2343 & 0 & 144 & 102 & 14396 \\
\hline novice -3 & 150 & 2337 & 0 & 93 & 0 & 15400 \\
\hline novice -41 & 115 & 2317 & 0 & 51 & 1000 & 14244 \\
\hline experienced-23 & 81 & 2306 & 189 & 119 & 1004 & 14214 \\
\hline novice-28 & 136 & 2221 & 0 & 120 & 0 & 13816 \\
\hline experienced-29 & 84 & 2214 & 59 & 133 & 1072 & 13566 \\
\hline novice-23 & 129 & 2138 & 0 & 72 & 0 & 13186 \\
\hline scientist-30 & 40 & 2129 & 186 & 123 & 409 & 13492 \\
\hline novice-31 & 157 & 2073 & 0 & 102 & 18 & 12692 \\
\hline novice-25 & 155 & 2066 & 2 & 217 & 0 & 13070 \\
\hline scientist-41 & 49 & 2037 & 0 & 36 & 0 & 13036 \\
\hline experienced-30 & 66 & 2028 & 82 & 110 & 624 & 12686 \\
\hline scientist-10 & 27 & 2024 & 77 & 120 & 730 & 12658 \\
\hline novice-37 & 154 & 1949 & 0 & 57 & 0 & 12044 \\
\hline
\end{tabular}




\begin{tabular}{|c|c|c|c|c|c|c|}
\hline novice-4 & 131 & 1919 & 0 & 123 & 0 & 11758 \\
\hline novice-22 & 166 & 1893 & 1 & 51 & 547 & 11844 \\
\hline experienced-34 & 76 & 1869 & 206 & 218 & 598 & 11676 \\
\hline scientist -1 & 13 & 1856 & 54 & 111 & 761 & 11792 \\
\hline non-11 & 98 & 1848 & $O$ & 61 & 0 & 12210 \\
\hline novice-8 & 153 & 1822 & 0 & 19 & 0 & 11298 \\
\hline experienced -14 & 88 & 1810 & 23 & 153 & 996 & 11270 \\
\hline experienced-19 & 58 & 1807 & 163 & 88 & 829 & 11328 \\
\hline experienced -12 & 77 & 1763 & 106 & 92 & 889 & 10840 \\
\hline scientist-21 & 19 & 1762 & 50 & 134 & 586 & 10894 \\
\hline scientist-39 & 41 & 1753 & 173 & 77 & 530 & 10992 \\
\hline experienced-27 & 67 & 1693 & 77 & 54 & 741 & 11032 \\
\hline novice -55 & 128 & 1662 & 6 & 40 & 0 & 10218 \\
\hline non-1 & 90 & 1622 & 0 & 59 & 410 & 10110 \\
\hline experienced-36 & 63 & 1580 & 56 & 116 & 781 & 9718 \\
\hline non-22 & 112 & 1567 & 48 & 56 & 0 & 10004 \\
\hline scientist -5 & 21 & 1563 & 18 & 78 & 558 & 10164 \\
\hline scientist-44 & 44 & 1543 & 12 & 84 & 394 & 9544 \\
\hline scientist -50 & 12 & 1496 & 219 & 225 & 387 & 9526 \\
\hline scientist-24 & 29 & 1494 & 0 & 55 & 1217 & 9250 \\
\hline experienced-25 & 83 & 1465 & 69 & 89 & 346 & 9072 \\
\hline novice-10 & 114 & 1464 & 0 & 40 & 872 & 9038 \\
\hline experienced-11 & 70 & 1456 & 21 & 86 & 927 & 9126 \\
\hline scientist -49 & 51 & 1448 & 138 & 97 & 179 & 9106 \\
\hline novice-35 & 135 & 1444 & 0 & 54 & 50 & 9022 \\
\hline scientist-15 & 50 & 1429 & 200 & 81 & 175 & 9216 \\
\hline non-18 & 111 & 1403 & 0 & 64 & 0 & 8804 \\
\hline novice -47 & 148 & 1316 & 0 & 78 & 0 & 8118 \\
\hline non-23 & 89 & 1294 & 0 & 48 & 636 & 8118 \\
\hline experienced-33 & 82 & 1292 & 83 & 65 & 649 & 7986 \\
\hline novice -44 & 158 & 1277 & 0 & 40 & 0 & 7896 \\
\hline novice-34 & 147 & 1276 & 4 & 46 & 0 & 8146 \\
\hline novice-2 & 160 & 1267 & 0 & 58 & 0 & 8072 \\
\hline non-3 & 108 & 1265 & 9 & 15 & 209 & 7928 \\
\hline non-7 & 101 & 1231 & 3 & 54 & 792 & 7704 \\
\hline novice-29 & 120 & 1230 & 0 & 44 & 0 & 7754 \\
\hline scientist -47 & 11 & 1229 & 9 & 81 & 618 & 7672 \\
\hline novice-27 & 139 & 1195 & 1 & 63 & 414 & 7452 \\
\hline novice-17 & 132 & 1194 & 0 & 59 & 0 & 7702 \\
\hline novice-15 & 141 & 1139 & 0 & 48 & 0 & 7148 \\
\hline novice-26 & 137 & 1120 & 0 & 60 & 0 & 7066 \\
\hline experienced -13 & 85 & 1109 & 25 & 160 & 446 & 6848 \\
\hline novice-39 & 163 & 1107 & 0 & 51 & 9 & 6936 \\
\hline scientist -6 & 14 & 1103 & 33 & 49 & 278 & 7196 \\
\hline novice-18 & 167 & 1088 & 0 & 38 & 0 & 6710 \\
\hline novice-42 & 119 & 1068 & 0 & 33 & 5 & 6774 \\
\hline scientist-35 & 22 & 1049 & 23 & 29 & 594 & 6612 \\
\hline novice-7 & 145 & 1039 & 98 & 51 & 36 & 6608 \\
\hline novice -53 & 124 & 1028 & 0 & 41 & 51 & 6558 \\
\hline novice-50 & 117 & 985 & 0 & 92 & 0 & 6100 \\
\hline scientist-26 & 6 & 983 & 0 & 70 & 231 & 6388 \\
\hline scientist-3 & 36 & 978 & 1 & 69 & 255 & 6398 \\
\hline experienced-32 & 54 & 974 & 47 & 87 & 303 & 6102 \\
\hline novice -40 & 165 & 967 & 0 & 24 & 722 & 6032 \\
\hline novice-30 & 149 & 946 & 0 & 28 & 0 & 5986 \\
\hline experienced -3 & 87 & 915 & 88 & 42 & 356 & 5600 \\
\hline scientist-51 & 34 & 910 & 0 & 67 & 358 & 5754 \\
\hline novice- 6 & 123 & 871 & 0 & 44 & 0 & 5520 \\
\hline scientist -45 & 35 & 862 & 17 & 59 & 223 & 5330 \\
\hline novice-9 & 134 & 853 & 0 & 63 & 0 & 5292 \\
\hline
\end{tabular}




\begin{tabular}{|c|c|c|c|c|c|c|}
\hline novice-21 & 127 & 849 & 1 & 42 & 0 & 5268 \\
\hline novice-24 & 130 & 849 & 48 & 53 & 118 & 5436 \\
\hline non-17 & 110 & 848 & 0 & 65 & $O$ & 5330 \\
\hline scientist -8 & 2 & 842 & 0 & 51 & 79 & 5294 \\
\hline novice-38 & 159 & 839 & 0 & 17 & 0 & 5468 \\
\hline non-16 & 105 & 821 & 144 & 26 & 0 & 5108 \\
\hline scientist-48 & 9 & 819 & 0 & 43 & 0 & 5216 \\
\hline experienced-16 & 60 & 795 & 24 & 22 & 245 & 4932 \\
\hline scientist-28 & 18 & 765 & 64 & 26 & 235 & 5032 \\
\hline experienced- 6 & 57 & 757 & 0 & 32 & 69 & 4752 \\
\hline scientist-22 & 7 & 750 & 0 & 39 & 20 & 5026 \\
\hline novice -49 & 156 & 723 & 0 & 31 & 0 & 4428 \\
\hline novice -54 & 138 & 683 & 0 & 56 & 0 & 4248 \\
\hline experienced-31 & 61 & 683 & 19 & 38 & 454 & 4368 \\
\hline experienced-26 & 72 & 679 & 0 & 66 & 59 & 4192 \\
\hline novice-13 & 168 & 652 & 0 & 49 & 0 & 4106 \\
\hline novice -45 & 116 & 651 & 0 & 16 & 0 & 4120 \\
\hline novice-52 & 140 & 650 & 0 & 38 & 0 & 4174 \\
\hline novice -43 & 125 & 608 & 0 & 26 & 45 & 3778 \\
\hline scientist-32 & 5 & 601 & 0 & 20 & 0 & 3916 \\
\hline novice-5 & 121 & 593 & 1 & 67 & 0 & 3804 \\
\hline experienced-18 & 69 & 575 & 5 & 21 & 114 & 3548 \\
\hline non-15 & 94 & 571 & 0 & 28 & 0 & 3736 \\
\hline scientist-17 & 46 & 569 & 0 & 38 & 0 & 3792 \\
\hline non-24 & 96 & 542 & 0 & 34 & 0 & 3390 \\
\hline non-10 & 93 & 495 & 0 & 20 & 0 & 3096 \\
\hline non-13 & 100 & 487 & $O$ & 5 & 0 & 3072 \\
\hline novice -51 & 143 & 480 & 0 & 20 & 0 & 3046 \\
\hline non-2 & 113 & 454 & 0 & 15 & 63 & 2934 \\
\hline experienced -10 & 56 & 446 & 2 & 26 & 170 & 2774 \\
\hline novice-20 & 144 & 418 & 5 & 19 & $O$ & 2722 \\
\hline novice-32 & 133 & 385 & 0 & 37 & 60 & 2512 \\
\hline scientist-7 & 33 & 366 & $O$ & 28 & 169 & 2246 \\
\hline non-9 & 102 & 357 & 4 & 23 & 45 & 2432 \\
\hline non-25 & 104 & 327 & 3 & 18 & 48 & 2264 \\
\hline scientist-16 & 8 & 326 & 0 & 29 & 38 & 2250 \\
\hline scientist-33 & 4 & 325 & 0 & 12 & 0 & 2044 \\
\hline novice -48 & 152 & 269 & 0 & 9 & 0 & 1704 \\
\hline novice-11 & 162 & 256 & 2 & 21 & 0 & 1770 \\
\hline novice-16 & 161 & 256 & 0 & 25 & 0 & 1598 \\
\hline scientist-31 & 1 & 250 & 9 & 20 & 12 & 1758 \\
\hline non-5 & 103 & 244 & 0 & 11 & 0 & 1770 \\
\hline non-8 & 97 & 239 & 28 & 13 & 18 & 1524 \\
\hline experienced-15 & 65 & 225 & 0 & 12 & 85 & 1404 \\
\hline experienced -2 & 64 & 219 & 6 & 11 & 33 & 1414 \\
\hline non-12 & 107 & 216 & 0 & 26 & 0 & 1390 \\
\hline scientist-11 & 31 & 205 & 0 & 13 & 0 & 1380 \\
\hline non-14 & 109 & 201 & 1 & 4 & 0 & 1272 \\
\hline non-6 & 95 & 177 & $O$ & 7 & 0 & 1152 \\
\hline non-19 & 92 & 175 & 0 & 7 & 116 & 1356 \\
\hline non-21 & 91 & 132 & 0 & 7 & 0 & 890 \\
\hline
\end{tabular}


Appendix B: Generated Statistics for the Greenberg Dataset (Ordered by History)

\begin{tabular}{|c|c|c|c|c|c|c|}
\hline User & Uid & Commands & History & Errors & Aliases & Lines \\
\hline scientist-36 & 20 & 12056 & 488 & 566 & 3161 & 73434 \\
\hline experienced-20 & 53 & 4556 & 435 & 370 & 1646 & 28054 \\
\hline scientist-13 & 42 & 3593 & 357 & 118 & 204 & 21988 \\
\hline experienced-22 & 73 & 2814 & 325 & 122 & 560 & 17478 \\
\hline scientist-2 & 25 & 2954 & 236 & 149 & 1656 & 18514 \\
\hline scientist-52 & 15 & 7705 & 231 & 299 & 717 & 47280 \\
\hline scientist-9 & 26 & 4067 & 224 & 65 & 665 & 25424 \\
\hline experienced-24 & 86 & 3331 & 222 & 228 & 1456 & 20440 \\
\hline scientist -50 & 12 & 1496 & 219 & 225 & 387 & 9526 \\
\hline experienced-34 & 76 & 1869 & 206 & 218 & 598 & 11676 \\
\hline scientist-15 & 50 & 1429 & 200 & 81 & 175 & 9216 \\
\hline experienced-23 & 81 & 2306 & 189 & 119 & 1004 & 14214 \\
\hline scientist-30 & 40 & 2129 & 186 & 123 & 409 & 13492 \\
\hline scientist-4 & 23 & 4507 & 178 & 320 & 789 & 27992 \\
\hline scientist-14 & 47 & 3433 & 178 & 183 & 2 & 21404 \\
\hline experienced-1 & 80 & 3714 & 174 & 298 & 1906 & 22830 \\
\hline scientist-39 & 41 & 1753 & 173 & 77 & 530 & 10992 \\
\hline non-20 & 99 & 4042 & 165 & 124 & 2122 & 24798 \\
\hline experienced -19 & 58 & 1807 & 163 & 88 & 829 & 11328 \\
\hline experienced-21 & 59 & 2394 & 157 & 83 & 974 & 14762 \\
\hline non-16 & 105 & 821 & 144 & 26 & 0 & 5108 \\
\hline scientist-49 & 51 & 1448 & 138 & 97 & 179 & 9106 \\
\hline scientist-37 & 32 & 4187 & 121 & 83 & 1866 & 25604 \\
\hline novice-46 & 146 & 4163 & 112 & 372 & 1909 & 26080 \\
\hline scientist-19 & 43 & 2831 & 106 & 112 & 1330 & 17560 \\
\hline experienced -12 & 77 & 1763 & 106 & 92 & 889 & 10840 \\
\hline scientist-27 & 37 & 3817 & 102 & 85 & 0 & 23344 \\
\hline novice-7 & 145 & 1039 & 98 & 51 & 36 & 6608 \\
\hline experienced-3 & 87 & 915 & 88 & 42 & 356 & 5600 \\
\hline experienced-9 & 71 & 2351 & 86 & 136 & 502 & 14500 \\
\hline experienced-33 & 82 & 1292 & 83 & 65 & 649 & 7986 \\
\hline experienced -30 & 66 & 2028 & 82 & 110 & 624 & 12686 \\
\hline scientist -46 & 3 & 2551 & 80 & 110 & 495 & 16480 \\
\hline experienced-28 & 74 & 3893 & 78 & 60 & 2516 & 25116 \\
\hline experienced-27 & 67 & 1693 & 77 & 54 & 741 & 11032 \\
\hline scientist-10 & 27 & 2024 & 77 & 120 & 730 & 12658 \\
\hline scientist-20 & 45 & 2697 & 74 & 189 & 804 & 17080 \\
\hline experienced-25 & 83 & 1465 & 69 & 89 & 346 & 9072 \\
\hline experienced-7 & 55 & 5857 & 67 & 612 & 2926 & 35896 \\
\hline experienced -8 & 68 & 2930 & 67 & 265 & 625 & 18114 \\
\hline scientist-28 & 18 & 765 & 64 & 26 & 235 & 5032 \\
\hline experienced-29 & 84 & 2214 & 59 & 133 & 1072 & 13566 \\
\hline experienced -36 & 63 & 1580 & 56 & 116 & 781 & 9718 \\
\hline scientist -1 & 13 & 1856 & 54 & 111 & 761 & 11792 \\
\hline scientist-12 & 28 & 2499 & 53 & 52 & 1162 & 15412 \\
\hline scientist-23 & 16 & 3360 & 52 & 135 & 481 & 21454 \\
\hline scientist-21 & 19 & 1762 & 50 & 134 & 586 & 10894 \\
\hline scientist-38 & 24 & 3775 & 48 & 168 & 1312 & 23172 \\
\hline non-22 & 112 & 1567 & 48 & 56 & 0 & 10004 \\
\hline novice-24 & 130 & 849 & 48 & 53 & 118 & 5436 \\
\hline experienced-32 & 54 & 974 & 47 & 87 & 303 & 6102 \\
\hline novice-1 & 151 & 2457 & 37 & 213 & 1381 & 14960 \\
\hline experienced -5 & 62 & 4015 & 35 & 222 & 910 & 25220 \\
\hline scientist-6 & 14 & 1103 & 33 & 49 & 278 & 7196 \\
\hline experienced-35 & 78 & 4272 & 28 & 169 & 2504 & 26290 \\
\hline non-8 & 97 & 239 & 28 & 13 & 18 & 1524 \\
\hline experienced -13 & 85 & 1109 & 25 & 160 & 446 & 6848 \\
\hline
\end{tabular}




\begin{tabular}{|c|c|c|c|c|c|c|}
\hline experienced-16 & 60 & 795 & 24 & 22 & 245 & 4932 \\
\hline scientist-35 & 22 & 1049 & 23 & 29 & 594 & 6612 \\
\hline experienced-14 & 88 & 1810 & 23 & 153 & 996 & 11270 \\
\hline experienced-11 & 70 & 1456 & 21 & 86 & 927 & 9126 \\
\hline scientist-29 & 17 & 2683 & 20 & 243 & 530 & 16632 \\
\hline experienced-31 & 61 & 683 & 19 & 38 & 454 & 4368 \\
\hline non-4 & 106 & 5050 & 18 & 161 & 3296 & 30830 \\
\hline scientist -5 & 21 & 1563 & 18 & 78 & 558 & 10164 \\
\hline scientist -45 & 35 & 862 & 17 & 59 & 223 & 5330 \\
\hline scientist-34 & 52 & 2639 & 15 & 88 & 910 & 16648 \\
\hline scientist-44 & 44 & 1543 & 12 & 84 & 394 & 9544 \\
\hline non-3 & 108 & 1265 & 9 & 15 & 209 & 7928 \\
\hline scientist -47 & 11 & 1229 & 9 & 81 & 618 & 7672 \\
\hline scientist-31 & 1 & 250 & 9 & 20 & 12 & 1758 \\
\hline scientist-25 & 30 & 3508 & 7 & 122 & 379 & 22706 \\
\hline novice-19 & 164 & 3401 & 7 & 363 & 0 & 20816 \\
\hline experienced-2 & 64 & 219 & 6 & 11 & 33 & 1414 \\
\hline novice -55 & 128 & 1662 & 6 & 40 & 0 & 10218 \\
\hline scientist-18 & 48 & 5584 & 6 & 240 & 1816 & 34258 \\
\hline scientist -42 & 10 & 6068 & 6 & 644 & 3243 & 37598 \\
\hline novice-20 & 144 & 418 & 5 & 19 & 0 & 2722 \\
\hline experienced-18 & 69 & 575 & 5 & 21 & 114 & 3548 \\
\hline non-9 & 102 & 357 & 4 & 23 & 45 & 2432 \\
\hline novice-34 & 147 & 1276 & 4 & 46 & 0 & 8146 \\
\hline non-25 & 104 & 327 & 3 & 18 & 48 & 2264 \\
\hline non-7 & 101 & 1231 & 3 & 54 & 792 & 7704 \\
\hline novice-25 & 155 & 2066 & 2 & 217 & 0 & 13070 \\
\hline novice-11 & 162 & 256 & 2 & 21 & 0 & 1770 \\
\hline experienced-4 & 75 & 3776 & 2 & 123 & 1329 & 23258 \\
\hline experienced-10 & 56 & 446 & 2 & 26 & 170 & 2774 \\
\hline non-14 & 109 & 201 & 1 & 4 & $O$ & 1272 \\
\hline novice-27 & 139 & 1195 & 1 & 63 & 414 & 7452 \\
\hline novice-21 & 127 & 849 & 1 & 42 & 0 & 5268 \\
\hline scientist -3 & 36 & 978 & 1 & 69 & 255 & 6398 \\
\hline novice-22 & 166 & 1893 & 1 & 51 & 547 & 11844 \\
\hline novice-5 & 121 & 593 & 1 & 67 & 0 & 3804 \\
\hline novice-9 & 134 & 853 & $O$ & 63 & 0 & 5292 \\
\hline novice-17 & 132 & 1194 & 0 & 59 & 0 & 7702 \\
\hline novice-23 & 129 & 2138 & 0 & 72 & 0 & 13186 \\
\hline novice -40 & 165 & 967 & 0 & 24 & 722 & 6032 \\
\hline scientist-26 & 6 & 983 & 0 & 70 & 231 & 6388 \\
\hline novice -4 & 131 & 1919 & 0 & 123 & 0 & 11758 \\
\hline novice-15 & 141 & 1139 & 0 & 48 & 0 & 7148 \\
\hline novice-32 & 133 & 385 & 0 & 37 & 60 & 2512 \\
\hline novice-54 & 138 & 683 & 0 & 56 & 0 & 4248 \\
\hline novice-26 & 137 & 1120 & 0 & 60 & 0 & 7066 \\
\hline novice-28 & 136 & 2221 & 0 & 120 & 0 & 13816 \\
\hline novice-35 & 135 & 1444 & 0 & 54 & 50 & 9022 \\
\hline novice-52 & 140 & 650 & 0 & 38 & 0 & 4174 \\
\hline novice-51 & 143 & 480 & 0 & 20 & 0 & 3046 \\
\hline scientist-32 & 5 & 601 & $O$ & 20 & 0 & 3916 \\
\hline novice -49 & 156 & 723 & 0 & 31 & 0 & 4428 \\
\hline novice-31 & 157 & 2073 & 0 & 102 & 18 & 12692 \\
\hline novice -44 & 158 & 1277 & 0 & 40 & 0 & 7896 \\
\hline novice-38 & 159 & 839 & $O$ & 17 & 0 & 5468 \\
\hline novice-2 & 160 & 1267 & 0 & 58 & 0 & 8072 \\
\hline novice-16 & 161 & 256 & 0 & 25 & 0 & 1598 \\
\hline novice-39 & 163 & 1107 & 0 & 51 & 9 & 6936 \\
\hline novice-18 & 167 & 1088 & 0 & 38 & 0 & 6710 \\
\hline novice-36 & 142 & 3213 & 0 & 137 & 0 & 19840 \\
\hline
\end{tabular}




\begin{tabular}{|c|c|c|c|c|c|c|}
\hline novice-37 & 154 & 1949 & 0 & 57 & 0 & 12044 \\
\hline scientist-33 & 4 & 325 & 0 & 12 & 0 & 2044 \\
\hline novice-14 & 126 & 3194 & 0 & 208 & 0 & 19786 \\
\hline novice -47 & 148 & 1316 & 0 & 78 & 0 & 8118 \\
\hline novice-30 & 149 & 946 & 0 & 28 & 0 & 5986 \\
\hline novice-3 & 150 & 2337 & 0 & 93 & 0 & 15400 \\
\hline scientist -8 & 2 & 842 & 0 & 51 & 79 & 5294 \\
\hline novice-48 & 152 & 269 & 0 & 9 & 0 & 1704 \\
\hline novice-8 & 153 & 1822 & $O$ & 19 & 0 & 11298 \\
\hline novice-13 & 168 & 652 & 0 & 49 & 0 & 4106 \\
\hline experienced-15 & 65 & 225 & 0 & 12 & 85 & 1404 \\
\hline scientist-24 & 29 & 1494 & 0 & 55 & 1217 & 9250 \\
\hline non-23 & 89 & 1294 & 0 & 48 & 636 & 8118 \\
\hline non-1 & 90 & 1622 & 0 & 59 & 410 & 10110 \\
\hline non-21 & 91 & 132 & 0 & 7 & 0 & 890 \\
\hline non-19 & 92 & 175 & 0 & 7 & 116 & 1356 \\
\hline non-10 & 93 & 495 & 0 & 20 & 0 & 3096 \\
\hline non-15 & 94 & 571 & 0 & 28 & 0 & 3736 \\
\hline non-6 & 95 & 177 & 0 & 7 & 0 & 1152 \\
\hline scientist-11 & 31 & 205 & 0 & 13 & 0 & 1380 \\
\hline scientist-7 & 33 & 366 & 0 & 28 & 169 & 2246 \\
\hline experienced-6 & 57 & 757 & 0 & 32 & 69 & 4752 \\
\hline scientist-41 & 49 & 2037 & $O$ & 36 & 0 & 13036 \\
\hline experienced-26 & 72 & 679 & 0 & 66 & 59 & 4192 \\
\hline scientist-17 & 46 & 569 & $O$ & 38 & 0 & 3792 \\
\hline scientist -43 & 39 & 3106 & 0 & 101 & 546 & 19066 \\
\hline scientist -40 & 38 & 4605 & 0 & 98 & 628 & 29020 \\
\hline experienced-17 & 79 & 2343 & 0 & 144 & 102 & 14396 \\
\hline scientist -51 & 34 & 910 & 0 & 67 & 358 & 5754 \\
\hline non-24 & 96 & 542 & 0 & 34 & 0 & 3390 \\
\hline non-11 & 98 & 1848 & 0 & 61 & 0 & 12210 \\
\hline scientist-48 & 9 & 819 & 0 & 43 & 0 & 5216 \\
\hline novice -45 & 116 & 651 & $O$ & 16 & 0 & 4120 \\
\hline novice -50 & 117 & 985 & 0 & 92 & 0 & 6100 \\
\hline novice-12 & 118 & 2436 & 0 & 210 & 0 & 16366 \\
\hline novice -42 & 119 & 1068 & 0 & 33 & 5 & 6774 \\
\hline novice-29 & 120 & 1230 & $O$ & 44 & 0 & 7754 \\
\hline novice-33 & 122 & 3127 & 0 & 106 & 0 & 19556 \\
\hline novice-6 & 123 & 871 & 0 & 44 & 0 & 5520 \\
\hline novice-53 & 124 & 1028 & 0 & 41 & 51 & 6558 \\
\hline novice-41 & 115 & 2317 & $O$ & 51 & 1000 & 14244 \\
\hline novice-10 & 114 & 1464 & 0 & 40 & 872 & 9038 \\
\hline non-13 & 100 & 487 & 0 & 5 & 0 & 3072 \\
\hline non-5 & 103 & 244 & 0 & 11 & 0 & 1770 \\
\hline scientist-16 & 8 & 326 & 0 & 29 & 38 & 2250 \\
\hline non-12 & 107 & 216 & 0 & 26 & 0 & 1390 \\
\hline non-17 & 110 & 848 & 0 & 65 & 0 & 5330 \\
\hline non-18 & 111 & 1403 & 0 & 64 & 0 & 8804 \\
\hline scientist-22 & 7 & 750 & 0 & 39 & 20 & 5026 \\
\hline non-2 & 113 & 454 & 0 & 15 & 63 & 2934 \\
\hline novice-43 & 125 & 608 & $O$ & 26 & 45 & 3778 \\
\hline
\end{tabular}


Appendix C: Generated Statistics for the Greenberg Dataset (Ordered by Errors)

\begin{tabular}{|c|c|c|c|c|c|c|}
\hline$U_{\text {ser }}$ & Uid & Commands & History & Errors & Aliases & Lines \\
\hline scientist-42 & 10 & 6068 & 6 & 644 & 3243 & 37598 \\
\hline experienced-7 & 55 & 5857 & 67 & 612 & 2926 & 35896 \\
\hline scientist-36 & 20 & 12056 & 488 & 566 & 3161 & 73434 \\
\hline novice-46 & 146 & 4163 & 112 & 372 & 1909 & 26080 \\
\hline experienced-20 & 53 & 4556 & 435 & 370 & 1646 & 28054 \\
\hline novice-19 & 164 & 3401 & 7 & 363 & 0 & 20816 \\
\hline scientist -4 & 23 & 4507 & 178 & 320 & 789 & 27992 \\
\hline scientist-52 & 15 & 7705 & 231 & 299 & 717 & 47280 \\
\hline experienced-1 & 80 & 3714 & 174 & 298 & 1906 & 22830 \\
\hline experienced -8 & 68 & 2930 & 67 & 265 & 625 & 18114 \\
\hline scientist-29 & 17 & 2683 & 20 & 243 & 530 & 16632 \\
\hline scientist-18 & 48 & 5584 & 6 & 240 & 1816 & 34258 \\
\hline experienced-24 & 86 & 3331 & 222 & 228 & 1456 & 20440 \\
\hline scientist -50 & 12 & 1496 & 219 & 225 & 387 & 9526 \\
\hline experienced -5 & 62 & 4015 & 35 & 222 & 910 & 25220 \\
\hline experienced-34 & 76 & 1869 & 206 & 218 & 598 & 11676 \\
\hline novice-25 & 155 & 2066 & 2 & 217 & 0 & 13070 \\
\hline novice-1 & 151 & 2457 & 37 & 213 & 1381 & 14960 \\
\hline novice-12 & 118 & 2436 & 0 & 210 & 0 & 16366 \\
\hline novice-14 & 126 & 3194 & 0 & 208 & 0 & 19786 \\
\hline scientist-20 & 45 & 2697 & 74 & 189 & 804 & 17080 \\
\hline scientist-14 & 47 & 3433 & 178 & 183 & 2 & 21404 \\
\hline experienced-35 & 78 & 4272 & 28 & 169 & 2504 & 26290 \\
\hline scientist-38 & 24 & 3775 & 48 & 168 & 1312 & 23172 \\
\hline non-4 & 106 & 5050 & 18 & 161 & 3296 & 30830 \\
\hline experienced -13 & 85 & 1109 & 25 & 160 & 446 & 6848 \\
\hline experienced-14 & 88 & 1810 & 23 & 153 & 996 & 11270 \\
\hline scientist-2 & 25 & 2954 & 236 & 149 & 1656 & 18514 \\
\hline experienced -17 & 79 & 2343 & 0 & 144 & 102 & 14396 \\
\hline novice-36 & 142 & 3213 & 0 & 137 & 0 & 19840 \\
\hline experienced -9 & 71 & 2351 & 86 & 136 & 502 & 14500 \\
\hline scientist-23 & 16 & 3360 & 52 & 135 & 481 & 21454 \\
\hline scientist-21 & 19 & 1762 & 50 & 134 & 586 & 10894 \\
\hline experienced-29 & 84 & 2214 & 59 & 133 & 1072 & 13566 \\
\hline non-20 & 99 & 4042 & 165 & 124 & 2122 & 24798 \\
\hline experienced -4 & 75 & 3776 & 2 & 123 & 1329 & 23258 \\
\hline novice-4 & 131 & 1919 & 0 & 123 & 0 & 11758 \\
\hline scientist-30 & 40 & 2129 & 186 & 123 & 409 & 13492 \\
\hline scientist-25 & 30 & 3508 & 7 & 122 & 379 & 22706 \\
\hline experienced-22 & 73 & 2814 & 325 & 122 & 560 & 17478 \\
\hline scientist-10 & 27 & 2024 & 77 & 120 & 730 & 12658 \\
\hline novice-28 & 136 & 2221 & 0 & 120 & 0 & 13816 \\
\hline experienced-23 & 81 & 2306 & 189 & 119 & 1004 & 14214 \\
\hline scientist-13 & 42 & 3593 & 357 & 118 & 204 & 21988 \\
\hline experienced-36 & 63 & 1580 & 56 & 116 & 781 & 9718 \\
\hline scientist-19 & 43 & 2831 & 106 & 112 & 1330 & 17560 \\
\hline scientist-1 & 13 & 1856 & 54 & 111 & 761 & 11792 \\
\hline experienced -30 & 66 & 2028 & 82 & 110 & 624 & 12686 \\
\hline scientist-46 & 3 & 2551 & 80 & 110 & 495 & 16480 \\
\hline novice-33 & 122 & 3127 & 0 & 106 & 0 & 19556 \\
\hline novice-31 & 157 & 2073 & 0 & 102 & 18 & 12692 \\
\hline scientist-43 & 39 & 3106 & 0 & 101 & 546 & 19066 \\
\hline scientist -40 & 38 & 4605 & 0 & 98 & 628 & 29020 \\
\hline scientist-49 & 51 & 1448 & 138 & 97 & 179 & 9106 \\
\hline novice-3 & 150 & 2337 & 0 & 93 & 0 & 15400 \\
\hline novice -50 & 117 & 985 & 0 & 92 & 0 & 6100 \\
\hline experienced-12 & 77 & 1763 & 106 & 92 & 889 & 10840 \\
\hline
\end{tabular}




\begin{tabular}{|c|c|c|c|c|c|c|}
\hline experienced-25 & 83 & 1465 & 69 & 89 & 346 & 9072 \\
\hline scientist-34 & 52 & 2639 & 15 & 88 & 910 & 16648 \\
\hline experienced-19 & 58 & 1807 & 163 & 88 & 829 & 11328 \\
\hline experienced-32 & 54 & 974 & 47 & 87 & 303 & 6102 \\
\hline experienced-11 & 70 & 1456 & 21 & 86 & 927 & 9126 \\
\hline scientist-27 & 37 & 3817 & 102 & 85 & 0 & 23344 \\
\hline scientist-44 & 44 & 1543 & 12 & 84 & 394 & 9544 \\
\hline scientist-37 & 32 & 4187 & 121 & 83 & 1866 & 25604 \\
\hline experienced-21 & 59 & 2394 & 157 & 83 & 974 & 14762 \\
\hline scientist -47 & 11 & 1229 & 9 & 81 & 618 & 7672 \\
\hline scientist-15 & 50 & 1429 & 200 & 81 & 175 & 9216 \\
\hline scientist -5 & 21 & 1563 & 18 & 78 & 558 & 10164 \\
\hline novice-47 & 148 & 1316 & 0 & 78 & 0 & 8118 \\
\hline scientist-39 & 41 & 1753 & 173 & 77 & 530 & 10992 \\
\hline novice-23 & 129 & 2138 & 0 & 72 & 0 & 13186 \\
\hline scientist-26 & 6 & 983 & 0 & 70 & 231 & 6388 \\
\hline scientist-3 & 36 & 978 & 1 & 69 & 255 & 6398 \\
\hline novice -5 & 121 & 593 & 1 & 67 & 0 & 3804 \\
\hline scientist-51 & 34 & 910 & 0 & 67 & 358 & 5754 \\
\hline experienced-26 & 72 & 679 & 0 & 66 & 59 & 4192 \\
\hline scientist-9 & 26 & 4067 & 224 & 65 & 665 & 25424 \\
\hline experienced-33 & 82 & 1292 & 83 & 65 & 649 & 7986 \\
\hline non-17 & 110 & 848 & 0 & 65 & 0 & 5330 \\
\hline non-18 & 111 & 1403 & 0 & 64 & 0 & 8804 \\
\hline novice-27 & 139 & 1195 & 1 & 63 & 414 & 7452 \\
\hline novice-9 & 134 & 853 & 0 & 63 & 0 & 5292 \\
\hline non-11 & 98 & 1848 & 0 & 61 & 0 & 12210 \\
\hline novice-26 & 137 & 1120 & 0 & 60 & 0 & 7066 \\
\hline experienced-28 & 74 & 3893 & 78 & 60 & 2516 & 25116 \\
\hline novice-17 & 132 & 1194 & 0 & 59 & 0 & 7702 \\
\hline scientist -45 & 35 & 862 & 17 & 59 & 223 & 5330 \\
\hline non-1 & 90 & 1622 & 0 & 59 & 410 & 10110 \\
\hline novice-2 & 160 & 1267 & 0 & 58 & 0 & 8072 \\
\hline novice-37 & 154 & 1949 & 0 & 57 & 0 & 12044 \\
\hline non-22 & 112 & 1567 & 48 & 56 & 0 & 10004 \\
\hline novice-54 & 138 & 683 & 0 & 56 & 0 & 4248 \\
\hline scientist-24 & 29 & 1494 & 0 & 55 & 1217 & 9250 \\
\hline non-7 & 101 & 1231 & 3 & 54 & 792 & 7704 \\
\hline experienced-27 & 67 & 1693 & 77 & 54 & 741 & 11032 \\
\hline novice-35 & 135 & 1444 & 0 & 54 & 50 & 9022 \\
\hline novice-24 & 130 & 849 & 48 & 53 & 118 & 5436 \\
\hline scientist-12 & 28 & 2499 & 53 & 52 & 1162 & 15412 \\
\hline novice-22 & 166 & 1893 & 1 & 51 & 547 & 11844 \\
\hline scientist -8 & 2 & 842 & 0 & 51 & 79 & 5294 \\
\hline novice-41 & 115 & 2317 & 0 & 51 & 1000 & 14244 \\
\hline novice-39 & 163 & 1107 & 0 & 51 & 9 & 6936 \\
\hline novice-7 & 145 & 1039 & 98 & 51 & 36 & 6608 \\
\hline novice-13 & 168 & 652 & 0 & 49 & 0 & 4106 \\
\hline scientist -6 & 14 & 1103 & 33 & 49 & 278 & 7196 \\
\hline non-23 & 89 & 1294 & 0 & 48 & 636 & 8118 \\
\hline novice-15 & 141 & 1139 & 0 & 48 & 0 & 7148 \\
\hline novice-34 & 147 & 1276 & 4 & 46 & 0 & 8146 \\
\hline novice-6 & 123 & 871 & $O$ & 44 & 0 & 5520 \\
\hline novice-29 & 120 & 1230 & 0 & 44 & 0 & 7754 \\
\hline scientist-48 & 9 & 819 & $O$ & 43 & 0 & 5216 \\
\hline experienced-3 & 87 & 915 & 88 & 42 & 356 & 5600 \\
\hline novice-21 & 127 & 849 & 1 & 42 & 0 & 5268 \\
\hline novice -53 & 124 & 1028 & 0 & 41 & 51 & 6558 \\
\hline novice-10 & 114 & 1464 & 0 & 40 & 872 & 9038 \\
\hline novice -44 & 158 & 1277 & 0 & 40 & 0 & 7896 \\
\hline
\end{tabular}




\begin{tabular}{|c|c|c|c|c|c|c|}
\hline novice-55 & 128 & 1662 & 6 & 40 & 0 & 10218 \\
\hline scientist-22 & 7 & 750 & 0 & 39 & 20 & 5026 \\
\hline experienced-31 & 61 & 683 & 19 & 38 & 454 & 4368 \\
\hline scientist-17 & 46 & 569 & 0 & 38 & 0 & 3792 \\
\hline novice-18 & 167 & 1088 & 0 & 38 & 0 & 6710 \\
\hline novice-52 & 140 & 650 & 0 & 38 & 0 & 4174 \\
\hline novice-32 & 133 & 385 & 0 & 37 & 60 & 2512 \\
\hline non-24 & 96 & 542 & 0 & 34 & 0 & 3390 \\
\hline novice-42 & 119 & 1068 & 0 & 33 & 5 & 6774 \\
\hline experienced- 6 & 57 & 757 & 0 & 32 & 69 & 4752 \\
\hline novice-49 & 156 & 723 & 0 & 31 & 0 & 4428 \\
\hline scientist-16 & 8 & 326 & 0 & 29 & 38 & 2250 \\
\hline scientist-35 & 22 & 1049 & 23 & 29 & 594 & 6612 \\
\hline scientist -7 & 33 & 366 & $O$ & 28 & 169 & 2246 \\
\hline non-15 & 94 & 571 & 0 & 28 & 0 & 3736 \\
\hline non-12 & 107 & 216 & 0 & 26 & 0 & 1390 \\
\hline scientist-28 & 18 & 765 & 64 & 26 & 235 & 5032 \\
\hline novice-43 & 125 & 608 & 0 & 26 & 45 & 3778 \\
\hline non-16 & 105 & 821 & 144 & 26 & 0 & 5108 \\
\hline experienced-10 & 56 & 446 & 2 & 26 & 170 & 2774 \\
\hline novice-16 & 161 & 256 & 0 & 25 & 0 & 1598 \\
\hline novice -40 & 165 & 967 & 0 & 24 & 722 & 6032 \\
\hline non-9 & 102 & 357 & 4 & 23 & 45 & 2432 \\
\hline experienced-16 & 60 & 795 & 24 & 22 & 245 & 4932 \\
\hline novice-11 & 162 & 256 & 2 & 21 & 0 & 1770 \\
\hline experienced-18 & 69 & 575 & 5 & 21 & 114 & 3548 \\
\hline non-10 & 93 & 495 & 0 & 20 & 0 & 3096 \\
\hline novice-51 & 143 & 480 & 0 & 20 & 0 & 3046 \\
\hline scientist-31 & 1 & 250 & 9 & 20 & 12 & 1758 \\
\hline novice-20 & 144 & 418 & 5 & 19 & $O$ & 2722 \\
\hline novice-8 & 153 & 1822 & 0 & 19 & 0 & 11298 \\
\hline non-25 & 104 & 327 & 3 & 18 & 48 & 2264 \\
\hline novice-38 & 159 & 839 & 0 & 17 & 0 & 5468 \\
\hline novice -45 & 116 & 651 & 0 & 16 & 0 & 4120 \\
\hline non-3 & 108 & 1265 & 9 & 15 & 209 & 7928 \\
\hline non-2 & 113 & 454 & 0 & 15 & 63 & 2934 \\
\hline scientist-11 & 31 & 205 & 0 & 13 & 0 & 1380 \\
\hline non-8 & 97 & 239 & 28 & 13 & 18 & 1524 \\
\hline experienced-15 & 65 & 225 & 0 & 12 & 85 & 1404 \\
\hline scientist-33 & 4 & 325 & 0 & 12 & $O$ & 2044 \\
\hline non-5 & 103 & 244 & 0 & 11 & 0 & 1770 \\
\hline experienced-2 & 64 & 219 & 6 & 11 & 33 & 1414 \\
\hline novice-48 & 152 & 269 & 0 & 9 & 0 & 1704 \\
\hline non-19 & 92 & 175 & 0 & 7 & 116 & 1356 \\
\hline non-6 & 95 & 177 & 0 & 7 & 0 & 1152 \\
\hline non-21 & 91 & 132 & 0 & 7 & 0 & 890 \\
\hline non-13 & 100 & 487 & 0 & 5 & 0 & 3072 \\
\hline non-14 & 109 & 201 & 1 & 4 & 0 & 1272 \\
\hline
\end{tabular}


Appendix D: Generated Statistics for the Greenberg Dataset (Ordered by Aliases)

\begin{tabular}{|c|c|c|c|c|c|c|}
\hline User & Uid & Commands & History & Errors & Aliases & Lines \\
\hline non-4 & 106 & 5050 & 18 & 161 & 3296 & 30830 \\
\hline scientist -42 & 10 & 6068 & 6 & 644 & 3243 & 37598 \\
\hline scientist-36 & 20 & 12056 & 488 & 566 & 3161 & 73434 \\
\hline experienced-7 & 55 & 5857 & 67 & 612 & 2926 & 35896 \\
\hline experienced-28 & 74 & 3893 & 78 & 60 & 2516 & 25116 \\
\hline experienced-35 & 78 & 4272 & 28 & 169 & 2504 & 26290 \\
\hline non-20 & 99 & 4042 & 165 & 124 & 2122 & 24798 \\
\hline novice-46 & 146 & 4163 & 112 & 372 & 1909 & 26080 \\
\hline experienced-1 & 80 & 3714 & 174 & 298 & 1906 & 22830 \\
\hline scientist-37 & 32 & 4187 & 121 & 83 & 1866 & 25604 \\
\hline scientist-18 & 48 & 5584 & 6 & 240 & 1816 & 34258 \\
\hline scientist-2 & 25 & 2954 & 236 & 149 & 1656 & 18514 \\
\hline experienced-20 & 53 & 4556 & 435 & 370 & 1646 & 28054 \\
\hline experienced-24 & 86 & 3331 & 222 & 228 & 1456 & 20440 \\
\hline novice-1 & 151 & 2457 & 37 & 213 & 1381 & 14960 \\
\hline scientist-19 & 43 & 2831 & 106 & 112 & 1330 & 17560 \\
\hline experienced -4 & 75 & 3776 & 2 & 123 & 1329 & 23258 \\
\hline scientist-38 & 24 & 3775 & 48 & 168 & 1312 & 23172 \\
\hline scientist-24 & 29 & 1494 & 0 & 55 & 1217 & 9250 \\
\hline scientist-12 & 28 & 2499 & 53 & 52 & 1162 & 15412 \\
\hline experienced-29 & 84 & 2214 & 59 & 133 & 1072 & 13566 \\
\hline experienced-23 & 81 & 2306 & 189 & 119 & 1004 & 14214 \\
\hline novice-41 & 115 & 2317 & 0 & 51 & 1000 & 14244 \\
\hline experienced -14 & 88 & 1810 & 23 & 153 & 996 & 11270 \\
\hline experienced-21 & 59 & 2394 & 157 & 83 & 974 & 14762 \\
\hline experienced-11 & 70 & 1456 & 21 & 86 & 927 & 9126 \\
\hline scientist-34 & 52 & 2639 & 15 & 88 & 910 & 16648 \\
\hline experienced -5 & 62 & 4015 & 35 & 222 & 910 & 25220 \\
\hline experienced-12 & 77 & 1763 & 106 & 92 & 889 & 10840 \\
\hline novice-10 & 114 & 1464 & $O$ & 40 & 872 & 9038 \\
\hline experienced -19 & 58 & 1807 & 163 & 88 & 829 & 11328 \\
\hline scientist-20 & 45 & 2697 & 74 & 189 & 804 & 17080 \\
\hline non-7 & 101 & 1231 & 3 & 54 & 792 & 7704 \\
\hline scientist-4 & 23 & 4507 & 178 & 320 & 789 & 27992 \\
\hline experienced-36 & 63 & 1580 & 56 & 116 & 781 & 9718 \\
\hline scientist-1 & 13 & 1856 & 54 & 111 & 761 & 11792 \\
\hline experienced-27 & 67 & 1693 & 77 & 54 & 741 & 11032 \\
\hline scientist -10 & 27 & 2024 & 77 & 120 & 730 & 12658 \\
\hline novice-40 & 165 & 967 & 0 & 24 & 722 & 6032 \\
\hline scientist-52 & 15 & 7705 & 231 & 299 & 717 & 47280 \\
\hline scientist-9 & 26 & 4067 & 224 & 65 & 665 & 25424 \\
\hline experienced-33 & 82 & 1292 & 83 & 65 & 649 & 7986 \\
\hline non-23 & 89 & 1294 & 0 & 48 & 636 & 8118 \\
\hline scientist-40 & 38 & 4605 & 0 & 98 & 628 & 29020 \\
\hline experienced-8 & 68 & 2930 & 67 & 265 & 625 & 18114 \\
\hline experienced -30 & 66 & 2028 & 82 & 110 & 624 & 12686 \\
\hline scientist -47 & 11 & 1229 & 9 & 81 & 618 & 7672 \\
\hline experienced-34 & 76 & 1869 & 206 & 218 & 598 & 11676 \\
\hline scientist-35 & 22 & 1049 & 23 & 29 & 594 & 6612 \\
\hline scientist-21 & 19 & 1762 & 50 & 134 & 586 & 10894 \\
\hline experienced-22 & 73 & 2814 & 325 & 122 & 560 & 17478 \\
\hline scientist -5 & 21 & 1563 & 18 & 78 & 558 & 10164 \\
\hline novice-22 & 166 & 1893 & 1 & 51 & 547 & 11844 \\
\hline scientist-43 & 39 & 3106 & 0 & 101 & 546 & 19066 \\
\hline scientist-29 & 17 & 2683 & 20 & 243 & 530 & 16632 \\
\hline scientist-39 & 41 & 1753 & 173 & 77 & 530 & 10992 \\
\hline experienced -9 & 71 & 2351 & 86 & 136 & 502 & 14500 \\
\hline
\end{tabular}




\begin{tabular}{|c|c|c|c|c|c|c|}
\hline scientist-46 & 3 & 2551 & 80 & 110 & 495 & 16480 \\
\hline scientist-23 & 16 & 3360 & 52 & 135 & 481 & 21454 \\
\hline experienced-31 & 61 & 683 & 19 & 38 & 454 & 4368 \\
\hline experienced-13 & 85 & 1109 & 25 & 160 & 446 & 6848 \\
\hline novice-27 & 139 & 1195 & 1 & 63 & 414 & 7452 \\
\hline non-1 & 90 & 1622 & 0 & 59 & 410 & 10110 \\
\hline scientist-30 & 40 & 2129 & 186 & 123 & 409 & 13492 \\
\hline scientist-44 & 44 & 1543 & 12 & 84 & 394 & 9544 \\
\hline scientist -50 & 12 & 1496 & 219 & 225 & 387 & 9526 \\
\hline scientist-25 & 30 & 3508 & 7 & 122 & 379 & 22706 \\
\hline scientist -51 & 34 & 910 & 0 & 67 & 358 & 5754 \\
\hline experienced-3 & 87 & 915 & 88 & 42 & 356 & 5600 \\
\hline experienced-25 & 83 & 1465 & 69 & 89 & 346 & 9072 \\
\hline experienced-32 & 54 & 974 & 47 & 87 & 303 & 6102 \\
\hline scientist- 6 & 14 & 1103 & 33 & 49 & 278 & 7196 \\
\hline scientist-3 & 36 & 978 & 1 & 69 & 255 & 6398 \\
\hline experienced-16 & 60 & 795 & 24 & 22 & 245 & 4932 \\
\hline scientist-28 & 18 & 765 & 64 & 26 & 235 & 5032 \\
\hline scientist-26 & 6 & 983 & 0 & 70 & 231 & 6388 \\
\hline scientist -45 & 35 & 862 & 17 & 59 & 223 & 5330 \\
\hline non-3 & 108 & 1265 & 9 & 15 & 209 & 7928 \\
\hline scientist-13 & 42 & 3593 & 357 & 118 & 204 & 21988 \\
\hline scientist-49 & 51 & 1448 & 138 & 97 & 179 & 9106 \\
\hline scientist-15 & 50 & 1429 & 200 & 81 & 175 & 9216 \\
\hline experienced -10 & 56 & 446 & 2 & 26 & 170 & 2774 \\
\hline scientist-7 & 33 & 366 & 0 & 28 & 169 & 2246 \\
\hline novice-24 & 130 & 849 & 48 & 53 & 118 & 5436 \\
\hline non-19 & 92 & 175 & 0 & 7 & 116 & 1356 \\
\hline experienced-18 & 69 & 575 & 5 & 21 & 114 & 3548 \\
\hline experienced-17 & 79 & 2343 & 0 & 144 & 102 & 14396 \\
\hline experienced-15 & 65 & 225 & 0 & 12 & 85 & 1404 \\
\hline scientist-8 & 2 & 842 & 0 & 51 & 79 & 5294 \\
\hline experienced-6 & 57 & 757 & $O$ & 32 & 69 & 4752 \\
\hline non-2 & 113 & 454 & 0 & 15 & 63 & 2934 \\
\hline novice-32 & 133 & 385 & 0 & 37 & 60 & 2512 \\
\hline experienced-26 & 72 & 679 & 0 & 66 & 59 & 4192 \\
\hline novice -53 & 124 & 1028 & $O$ & 41 & 51 & 6558 \\
\hline novice-35 & 135 & 1444 & 0 & 54 & 50 & 9022 \\
\hline non-25 & 104 & 327 & 3 & 18 & 48 & 2264 \\
\hline non-9 & 102 & 357 & 4 & 23 & 45 & 2432 \\
\hline novice-43 & 125 & 608 & 0 & 26 & 45 & 3778 \\
\hline scientist-16 & 8 & 326 & 0 & 29 & 38 & 2250 \\
\hline novice-7 & 145 & 1039 & 98 & 51 & 36 & 6608 \\
\hline experienced-2 & 64 & 219 & 6 & 11 & 33 & 1414 \\
\hline scientist-22 & 7 & 750 & 0 & 39 & 20 & 5026 \\
\hline non-8 & 97 & 239 & 28 & 13 & 18 & 1524 \\
\hline novice-31 & 157 & 2073 & 0 & 102 & 18 & 12692 \\
\hline scientist-31 & 1 & 250 & 9 & 20 & 12 & 1758 \\
\hline novice-39 & 163 & 1107 & 0 & 51 & 9 & 6936 \\
\hline novice -42 & 119 & 1068 & 0 & 33 & 5 & 6774 \\
\hline scientist-14 & 47 & 3433 & 178 & 183 & 2 & 21404 \\
\hline novice-30 & 149 & 946 & 0 & 28 & 0 & 5986 \\
\hline novice-47 & 148 & 1316 & $O$ & 78 & 0 & 8118 \\
\hline novice-3 & 150 & 2337 & 0 & 93 & 0 & 15400 \\
\hline novice-34 & 147 & 1276 & 4 & 46 & 0 & 8146 \\
\hline scientist-17 & 46 & 569 & 0 & 38 & 0 & 3792 \\
\hline novice-20 & 144 & 418 & 5 & 19 & 0 & 2722 \\
\hline novice-51 & 143 & 480 & 0 & 20 & 0 & 3046 \\
\hline novice-36 & 142 & 3213 & 0 & 137 & 0 & 19840 \\
\hline novice-15 & 141 & 1139 & 0 & 48 & 0 & 7148 \\
\hline
\end{tabular}




\begin{tabular}{|c|c|c|c|c|c|c|}
\hline novice-52 & 140 & 650 & 0 & 38 & 0 & 4174 \\
\hline scientist-27 & 37 & 3817 & 102 & 85 & 0 & 23344 \\
\hline scientist-32 & 5 & 601 & 0 & 20 & 0 & 3916 \\
\hline novice -48 & 152 & 269 & 0 & 9 & 0 & 1704 \\
\hline novice-8 & 153 & 1822 & 0 & 19 & 0 & 11298 \\
\hline novice-13 & 168 & 652 & 0 & 49 & 0 & 4106 \\
\hline novice-18 & 167 & 1088 & 0 & 38 & 0 & 6710 \\
\hline scientist-41 & 49 & 2037 & 0 & 36 & 0 & 13036 \\
\hline scientist-33 & 4 & 325 & 0 & 12 & 0 & 2044 \\
\hline novice-19 & 164 & 3401 & 7 & 363 & 0 & 20816 \\
\hline novice-11 & 162 & 256 & 2 & 21 & 0 & 1770 \\
\hline novice -16 & 161 & 256 & 0 & 25 & 0 & 1598 \\
\hline novice-2 & 160 & 1267 & 0 & 58 & 0 & 8072 \\
\hline novice-38 & 159 & 839 & 0 & 17 & 0 & 5468 \\
\hline novice-44 & 158 & 1277 & 0 & 40 & 0 & 7896 \\
\hline novice -49 & 156 & 723 & 0 & 31 & 0 & 4428 \\
\hline novice-25 & 155 & 2066 & 2 & 217 & 0 & 13070 \\
\hline novice-37 & 154 & 1949 & 0 & 57 & 0 & 12044 \\
\hline novice-54 & 138 & 683 & 0 & 56 & 0 & 4248 \\
\hline novice-26 & 137 & 1120 & 0 & 60 & 0 & 7066 \\
\hline novice-28 & 136 & 2221 & 0 & 120 & 0 & 13816 \\
\hline novice-12 & 118 & 2436 & 0 & 210 & 0 & 16366 \\
\hline novice-50 & 117 & 985 & 0 & 92 & 0 & 6100 \\
\hline novice -45 & 116 & 651 & 0 & 16 & 0 & 4120 \\
\hline non-6 & 95 & 177 & 0 & 7 & 0 & 1152 \\
\hline non-24 & 96 & 542 & 0 & 34 & 0 & 3390 \\
\hline non-22 & 112 & 1567 & 48 & 56 & 0 & 10004 \\
\hline non-18 & 111 & 1403 & 0 & 64 & 0 & 8804 \\
\hline non-17 & 110 & 848 & 0 & 65 & 0 & 5330 \\
\hline non-14 & 109 & 201 & 1 & 4 & 0 & 1272 \\
\hline non-11 & 98 & 1848 & 0 & 61 & 0 & 12210 \\
\hline non-12 & 107 & 216 & 0 & 26 & 0 & 1390 \\
\hline non-13 & 100 & 487 & $O$ & 5 & 0 & 3072 \\
\hline non-16 & 105 & 821 & 144 & 26 & 0 & 5108 \\
\hline non-5 & 103 & 244 & 0 & 11 & 0 & 1770 \\
\hline novice-29 & 120 & 1230 & 0 & 44 & 0 & 7754 \\
\hline novice -5 & 121 & 593 & 1 & 67 & 0 & 3804 \\
\hline scientist-11 & 31 & 205 & 0 & 13 & 0 & 1380 \\
\hline novice-9 & 134 & 853 & 0 & 63 & 0 & 5292 \\
\hline novice-17 & 132 & 1194 & 0 & 59 & 0 & 7702 \\
\hline novice-4 & 131 & 1919 & 0 & 123 & 0 & 11758 \\
\hline non-21 & 91 & 132 & 0 & 7 & 0 & 890 \\
\hline novice-23 & 129 & 2138 & 0 & 72 & 0 & 13186 \\
\hline novice-55 & 128 & 1662 & 6 & 40 & 0 & 10218 \\
\hline novice-21 & 127 & 849 & 1 & 42 & 0 & 5268 \\
\hline novice-14 & 126 & 3194 & 0 & 208 & 0 & 19786 \\
\hline non-10 & 93 & 495 & 0 & 20 & 0 & 3096 \\
\hline non-15 & 94 & 571 & 0 & 28 & 0 & 3736 \\
\hline novice-6 & 123 & 871 & 0 & 44 & 0 & 5520 \\
\hline novice-33 & 122 & 3127 & 0 & 106 & 0 & 19556 \\
\hline scientist-48 & 9 & 819 & 0 & 43 & 0 & 5216 \\
\hline
\end{tabular}


Appendix E: Generated Statistics for the Greenberg Dataset (Ordered by Lines)

\begin{tabular}{|c|c|c|c|c|c|c|}
\hline User & Uid & Commands & History & Errors & Aliases & Lines \\
\hline scientist-36 & 20 & 12056 & 488 & 566 & 3161 & 73434 \\
\hline scientist-52 & 15 & 7705 & 231 & 299 & 717 & 47280 \\
\hline scientist- 42 & 10 & 6068 & 6 & 644 & 3243 & 37598 \\
\hline experienced -7 & 55 & 5857 & 67 & 612 & 2926 & 35896 \\
\hline scientist-18 & 48 & 5584 & 6 & 240 & 1816 & 34258 \\
\hline non-4 & 106 & 5050 & 18 & 161 & 3296 & 30830 \\
\hline scientist -40 & 38 & 4605 & 0 & 98 & 628 & 29020 \\
\hline experienced-20 & 53 & 4556 & 435 & 370 & 1646 & 28054 \\
\hline scientist -4 & 23 & 4507 & 178 & 320 & 789 & 27992 \\
\hline experienced-35 & 78 & 4272 & 28 & 169 & 2504 & 26290 \\
\hline novice -46 & 146 & 4163 & 112 & 372 & 1909 & 26080 \\
\hline scientist-37 & 32 & 4187 & 121 & 83 & 1866 & 25604 \\
\hline scientist-9 & 26 & 4067 & 224 & 65 & 665 & 25424 \\
\hline experienced -5 & 62 & 4015 & 35 & 222 & 910 & 25220 \\
\hline experienced-28 & 74 & 3893 & 78 & 60 & 2516 & 25116 \\
\hline non-20 & 99 & 4042 & 165 & 124 & 2122 & 24798 \\
\hline scientist-27 & 37 & 3817 & 102 & 85 & 0 & 23344 \\
\hline experienced -4 & 75 & 3776 & 2 & 123 & 1329 & 23258 \\
\hline scientist-38 & 24 & 3775 & 48 & 168 & 1312 & 23172 \\
\hline experienced-1 & 80 & 3714 & 174 & 298 & 1906 & 22830 \\
\hline scientist-25 & 30 & 3508 & 7 & 122 & 379 & 22706 \\
\hline scientist-13 & 42 & 3593 & 357 & 118 & 204 & 21988 \\
\hline scientist-23 & 16 & 3360 & 52 & 135 & 481 & 21454 \\
\hline scientist-14 & 47 & 3433 & 178 & 183 & 2 & 21404 \\
\hline novice-19 & 164 & 3401 & 7 & 363 & 0 & 20816 \\
\hline experienced-24 & 86 & 3331 & 222 & 228 & 1456 & 20440 \\
\hline novice-36 & 142 & 3213 & 0 & 137 & 0 & 19840 \\
\hline novice-14 & 126 & 3194 & 0 & 208 & 0 & 19786 \\
\hline novice-33 & 122 & 3127 & 0 & 106 & 0 & 19556 \\
\hline scientist -43 & 39 & 3106 & 0 & 101 & 546 & 19066 \\
\hline scientist-2 & 25 & 2954 & 236 & 149 & 1656 & 18514 \\
\hline experienced -8 & 68 & 2930 & 67 & 265 & 625 & 18114 \\
\hline scientist-19 & 43 & 2831 & 106 & 112 & 1330 & 17560 \\
\hline experienced-22 & 73 & 2814 & 325 & 122 & 560 & 17478 \\
\hline scientist-20 & 45 & 2697 & 74 & 189 & 804 & 17080 \\
\hline scientist-34 & 52 & 2639 & 15 & 88 & 910 & 16648 \\
\hline scientist-29 & 17 & 2683 & 20 & 243 & 530 & 16632 \\
\hline scientist -46 & 3 & 2551 & 80 & 110 & 495 & 16480 \\
\hline novice-12 & 118 & 2436 & 0 & 210 & 0 & 16366 \\
\hline scientist-12 & 28 & 2499 & 53 & 52 & 1162 & 15412 \\
\hline novice-3 & 150 & 2337 & 0 & 93 & 0 & 15400 \\
\hline novice-1 & 151 & 2457 & 37 & 213 & 1381 & 14960 \\
\hline experienced-21 & 59 & 2394 & 157 & 83 & 974 & 14762 \\
\hline experienced-9 & 71 & 2351 & 86 & 136 & 502 & 14500 \\
\hline experienced-17 & 79 & 2343 & 0 & 144 & 102 & 14396 \\
\hline novice -41 & 115 & 2317 & 0 & 51 & 1000 & 14244 \\
\hline experienced-23 & 81 & 2306 & 189 & 119 & 1004 & 14214 \\
\hline novice-28 & 136 & 2221 & 0 & 120 & 0 & 13816 \\
\hline experienced-29 & 84 & 2214 & 59 & 133 & 1072 & 13566 \\
\hline scientist-30 & 40 & 2129 & 186 & 123 & 409 & 13492 \\
\hline novice-23 & 129 & 2138 & 0 & 72 & 0 & 13186 \\
\hline novice-25 & 155 & 2066 & 2 & 217 & 0 & 13070 \\
\hline scientist -41 & 49 & 2037 & 0 & 36 & 0 & 13036 \\
\hline novice-31 & 157 & 2073 & 0 & 102 & 18 & 12692 \\
\hline experienced -30 & 66 & 2028 & 82 & 110 & 624 & 12686 \\
\hline scientist-10 & 27 & 2024 & 77 & 120 & 730 & 12658 \\
\hline non-11 & 98 & 1848 & 0 & 61 & 0 & 12210 \\
\hline
\end{tabular}




\begin{tabular}{|c|c|c|c|c|c|c|}
\hline novice-37 & 154 & 1949 & $O$ & 57 & 0 & 12044 \\
\hline novice-22 & 166 & 1893 & 1 & 51 & 547 & 11844 \\
\hline scientist-1 & 13 & 1856 & 54 & 111 & 761 & 11792 \\
\hline novice-4 & 131 & 1919 & 0 & 123 & 0 & 11758 \\
\hline experienced-34 & 76 & 1869 & 206 & 218 & 598 & 11676 \\
\hline experienced-19 & 58 & 1807 & 163 & 88 & 829 & 11328 \\
\hline novice-8 & 153 & 1822 & 0 & 19 & 0 & 11298 \\
\hline experienced-14 & 88 & 1810 & 23 & 153 & 996 & 11270 \\
\hline experienced-27 & 67 & 1693 & 77 & 54 & 741 & 11032 \\
\hline scientist-39 & 41 & 1753 & 173 & 77 & 530 & 10992 \\
\hline scientist-21 & 19 & 1762 & 50 & 134 & 586 & 10894 \\
\hline experienced-12 & 77 & 1763 & 106 & 92 & 889 & 10840 \\
\hline novice-55 & 128 & 1662 & 6 & 40 & $O$ & 10218 \\
\hline scientist -5 & 21 & 1563 & 18 & 78 & 558 & 10164 \\
\hline non-1 & 90 & 1622 & 0 & 59 & 410 & 10110 \\
\hline non-22 & 112 & 1567 & 48 & 56 & 0 & 10004 \\
\hline experienced-36 & 63 & 1580 & 56 & 116 & 781 & 9718 \\
\hline scientist-44 & 44 & 1543 & 12 & 84 & 394 & 9544 \\
\hline scientist -50 & 12 & 1496 & 219 & 225 & 387 & 9526 \\
\hline scientist-24 & 29 & 1494 & 0 & 55 & 1217 & 9250 \\
\hline scientist-15 & 50 & 1429 & 200 & 81 & 175 & 9216 \\
\hline experienced-11 & 70 & 1456 & 21 & 86 & 927 & 9126 \\
\hline scientist -49 & 51 & 1448 & 138 & 97 & 179 & 9106 \\
\hline experienced-25 & 83 & 1465 & 69 & 89 & 346 & 9072 \\
\hline novice-10 & 114 & 1464 & 0 & 40 & 872 & 9038 \\
\hline novice-35 & 135 & 1444 & 0 & 54 & 50 & 9022 \\
\hline non-18 & 111 & 1403 & 0 & 64 & 0 & 8804 \\
\hline novice-34 & 147 & 1276 & 4 & 46 & 0 & 8146 \\
\hline novice -47 & 148 & 1316 & 0 & 78 & 0 & 8118 \\
\hline non-23 & 89 & 1294 & 0 & 48 & 636 & 8118 \\
\hline novice-2 & 160 & 1267 & 0 & 58 & 0 & 8072 \\
\hline experienced-33 & 82 & 1292 & 83 & 65 & 649 & 7986 \\
\hline non-3 & 108 & 1265 & 9 & 15 & 209 & 7928 \\
\hline novice-44 & 158 & 1277 & 0 & 40 & 0 & 7896 \\
\hline novice-29 & 120 & 1230 & 0 & 44 & 0 & 7754 \\
\hline non-7 & 101 & 1231 & 3 & 54 & 792 & 7704 \\
\hline novice-17 & 132 & 1194 & 0 & 59 & 0 & 7702 \\
\hline scientist -47 & 11 & 1229 & 9 & 81 & 618 & 7672 \\
\hline novice-27 & 139 & 1195 & 1 & 63 & 414 & 7452 \\
\hline scientist -6 & 14 & 1103 & 33 & 49 & 278 & 7196 \\
\hline novice-15 & 141 & 1139 & 0 & 48 & $O$ & 7148 \\
\hline novice-26 & 137 & 1120 & 0 & 60 & 0 & 7066 \\
\hline novice-39 & 163 & 1107 & 0 & 51 & 9 & 6936 \\
\hline experienced-13 & 85 & 1109 & 25 & 160 & 446 & 6848 \\
\hline novice -42 & 119 & 1068 & 0 & 33 & 5 & 6774 \\
\hline novice-18 & 167 & 1088 & 0 & 38 & 0 & 6710 \\
\hline scientist-35 & 22 & 1049 & 23 & 29 & 594 & 6612 \\
\hline novice-7 & 145 & 1039 & 98 & 51 & 36 & 6608 \\
\hline novice-53 & 124 & 1028 & 0 & 41 & 51 & 6558 \\
\hline scientist -3 & 36 & 978 & 1 & 69 & 255 & 6398 \\
\hline scientist-26 & 6 & 983 & 0 & 70 & 231 & 6388 \\
\hline experienced-32 & 54 & 974 & 47 & 87 & 303 & 6102 \\
\hline novice-50 & 117 & 985 & 0 & 92 & 0 & 6100 \\
\hline novice -40 & 165 & 967 & 0 & 24 & 722 & 6032 \\
\hline novice-30 & 149 & 946 & 0 & 28 & $O$ & 5986 \\
\hline scientist-51 & 34 & 910 & 0 & 67 & 358 & 5754 \\
\hline experienced-3 & 87 & 915 & 88 & 42 & 356 & 5600 \\
\hline novice-6 & 123 & 871 & 0 & 44 & 0 & 5520 \\
\hline novice-38 & 159 & 839 & 0 & 17 & 0 & 5468 \\
\hline novice-24 & 130 & 849 & 48 & 53 & 118 & 5436 \\
\hline
\end{tabular}




\begin{tabular}{|c|c|c|c|c|c|c|}
\hline scientist-45 & 35 & 862 & 17 & 59 & 223 & 5330 \\
\hline non-17 & 110 & 848 & 0 & 65 & 0 & 5330 \\
\hline scientist -8 & 2 & 842 & 0 & 51 & 79 & 5294 \\
\hline novice-9 & 134 & 853 & 0 & 63 & 0 & 5292 \\
\hline novice-21 & 127 & 849 & 1 & 42 & 0 & 5268 \\
\hline scientist -48 & 9 & 819 & 0 & 43 & 0 & 5216 \\
\hline non-16 & 105 & 821 & 144 & 26 & 0 & 5108 \\
\hline scientist-28 & 18 & 765 & 64 & 26 & 235 & 5032 \\
\hline scientist-22 & 7 & 750 & 0 & 39 & 20 & 5026 \\
\hline experienced-16 & 60 & 795 & 24 & 22 & 245 & 4932 \\
\hline experienced-6 & 57 & 757 & 0 & 32 & 69 & 4752 \\
\hline novice -49 & 156 & 723 & 0 & 31 & 0 & 4428 \\
\hline experienced-31 & 61 & 683 & 19 & 38 & 454 & 4368 \\
\hline novice -54 & 138 & 683 & 0 & 56 & 0 & 4248 \\
\hline experienced-26 & 72 & 679 & 0 & 66 & 59 & 4192 \\
\hline novice -52 & 140 & 650 & 0 & 38 & 0 & 4174 \\
\hline novice -45 & 116 & 651 & 0 & 16 & 0 & 4120 \\
\hline novice-13 & 168 & 652 & 0 & 49 & 0 & 4106 \\
\hline scientist-32 & 5 & 601 & 0 & 20 & 0 & 3916 \\
\hline novice -5 & 121 & 593 & 1 & 67 & 0 & 3804 \\
\hline scientist-17 & 46 & 569 & 0 & 38 & 0 & 3792 \\
\hline novice-43 & 125 & 608 & 0 & 26 & 45 & 3778 \\
\hline non-15 & 94 & 571 & 0 & 28 & 0 & 3736 \\
\hline experienced-18 & 69 & 575 & 5 & 21 & 114 & 3548 \\
\hline non-24 & 96 & 542 & 0 & 34 & 0 & 3390 \\
\hline non-10 & 93 & 495 & 0 & 20 & 0 & 3096 \\
\hline non-13 & 100 & 487 & 0 & 5 & 0 & 3072 \\
\hline novice-51 & 143 & 480 & 0 & 20 & 0 & 3046 \\
\hline non-2 & 113 & 454 & 0 & 15 & 63 & 2934 \\
\hline experienced-10 & 56 & 446 & 2 & 26 & 170 & 2774 \\
\hline novice-20 & 144 & 418 & 5 & 19 & $O$ & 2722 \\
\hline novice-32 & 133 & 385 & 0 & 37 & 60 & 2512 \\
\hline non-9 & 102 & 357 & 4 & 23 & 45 & 2432 \\
\hline non-25 & 104 & 327 & 3 & 18 & 48 & 2264 \\
\hline scientist-16 & 8 & 326 & 0 & 29 & 38 & 2250 \\
\hline scientist-7 & 33 & 366 & 0 & 28 & 169 & 2246 \\
\hline scientist-33 & 4 & 325 & 0 & 12 & 0 & 2044 \\
\hline non-5 & 103 & 244 & 0 & 11 & 0 & 1770 \\
\hline novice-11 & 162 & 256 & 2 & 21 & 0 & 1770 \\
\hline scientist-31 & 1 & 250 & 9 & 20 & 12 & 1758 \\
\hline novice -48 & 152 & 269 & 0 & 9 & 0 & 1704 \\
\hline novice-16 & 161 & 256 & 0 & 25 & 0 & 1598 \\
\hline non-8 & 97 & 239 & 28 & 13 & 18 & 1524 \\
\hline experienced-2 & 64 & 219 & 6 & 11 & 33 & 1414 \\
\hline experienced-15 & 65 & 225 & 0 & 12 & 85 & 1404 \\
\hline non-12 & 107 & 216 & 0 & 26 & 0 & 1390 \\
\hline scientist-11 & 31 & 205 & 0 & 13 & 0 & 1380 \\
\hline non-19 & 92 & 175 & 0 & 7 & 116 & 1356 \\
\hline non-14 & 109 & 201 & 1 & 4 & 0 & 1272 \\
\hline non- 6 & 95 & 177 & 0 & 7 & 0 & 1152 \\
\hline non-21 & 91 & 132 & 0 & 7 & 0 & 890 \\
\hline
\end{tabular}


Appendix F: List of User Profiles (Victims)

\begin{tabular}{|c|c|c|c|}
\hline Uid & $\begin{array}{c}\text { Number of } \\
\text { Distinct } \\
\text { Commands }\end{array}$ & Uid & $\begin{array}{c}\text { Number of } \\
\text { Distinct } \\
\text { Commnds }\end{array}$ \\
\hline 3 & 427 & 73 & 234 \\
\hline 10 & 427 & 74 & 350 \\
\hline 13 & 425 & 75 & 193 \\
\hline 15 & 316 & 76 & 244 \\
\hline 16 & 273 & 77 & 336 \\
\hline 17 & 507 & 78 & 241 \\
\hline 19 & 412 & 79 & 338 \\
\hline 20 & 376 & 80 & 241 \\
\hline 21 & 345 & 81 & 188 \\
\hline 23 & 351 & 84 & 230 \\
\hline 24 & 426 & 86 & 420 \\
\hline 25 & 286 & 88 & 274 \\
\hline 26 & 311 & 90 & 341 \\
\hline 27 & 312 & 98 & 472 \\
\hline 28 & 314 & 99 & 262 \\
\hline 30 & 462 & 106 & 322 \\
\hline 32 & 320 & 112 & 277 \\
\hline 37 & 275 & 115 & 252 \\
\hline 38 & 329 & 118 & 138 \\
\hline 39 & 349 & 122 & 138 \\
\hline 40 & 389 & 126 & 358 \\
\hline 41 & 342 & 128 & 137 \\
\hline 42 & 222 & 129 & 126 \\
\hline 43 & 307 & 131 & 255 \\
\hline 44 & 367 & 136 & 195 \\
\hline 45 & 422 & 142 & 157 \\
\hline 47 & 334 & 146 & 339 \\
\hline 48 & 314 & 150 & 119 \\
\hline 49 & 267 & 151 & 284 \\
\hline 52 & 214 & 153 & 107 \\
\hline 53 & 168 & 154 & 230 \\
\hline 55 & 339 & 155 & 339 \\
\hline 58 & 321 & 157 & 129 \\
\hline 59 & 195 & 164 & 206 \\
\hline 62 & 267 & 166 & 208 \\
\hline 63 & 274 & & \\
\hline 66 & 204 & & \\
\hline 67 & 341 & & \\
\hline 68 & 335 & & \\
\hline 71 & 341 & & \\
\hline
\end{tabular}




\section{Appendix G: Undecided Results}

\begin{tabular}{|c|c|c|c|c|c|c|c|}
\hline Uid Input & Uid Profile & Decision Expected & Interval Size & $p_{1}$ & $p_{2}$ & $\alpha$ & $\beta$ \\
\hline 70 & 52 & Reject & 10 & 0.29 & 0.71 & 0.01 & 0.01 \\
\hline 22 & 52 & Reject & 10 & 0.29 & 0.71 & 0.01 & 0.01 \\
\hline 82 & 52 & Reject & 10 & 0.29 & 0.71 & 0.01 & 0.01 \\
\hline 70 & 52 & Reject & 10 & 0.30 & 0.70 & 0.01 & 0.01 \\
\hline 22 & 52 & Reject & 10 & 0.30 & 0.70 & 0.01 & 0.01 \\
\hline 82 & 52 & Reject & 10 & 0.30 & 0.70 & 0.01 & 0.01 \\
\hline 70 & 84 & Reject & 10 & 0.31 & 0.69 & 0.01 & 0.01 \\
\hline 22 & 84 & Reject & 10 & 0.31 & 0.69 & 0.01 & 0.01 \\
\hline 82 & 84 & Reject & 10 & 0.31 & 0.69 & 0.01 & 0.01 \\
\hline 13 & 13 & Accept & 15 & 0.29 & 0.71 & 0.01 & 0.01 \\
\hline 13 & 13 & Accept & 20 & 0.31 & 0.69 & 0.01 & 0.01 \\
\hline 13 & 13 & Accept & 15 & 0.30 & 0.7 & 0.01 & 0.01 \\
\hline 13 & 13 & Accept & 15 & 0.30 & 0.7 & 0.01 & 0.01 \\
\hline 13 & 13 & Accept & 20 & 0.29 & 0.71 & 0.01 & 0.01 \\
\hline 13 & 13 & Accept & 10 & 0.31 & 0.69 & 0.01 & 0.01 \\
\hline 13 & 13 & Accept & 10 & 0.30 & 0.7 & 0.01 & 0.01 \\
\hline 13 & 13 & Accept & 20 & 0.30 & 0.7 & 0.01 & 0.01 \\
\hline 30 & 30 & Accept & 15 & 0.31 & 0.69 & 0.01 & 0.01 \\
\hline 30 & 30 & Accept & 5 & 0.45 & 0.55 & 0.01 & 0.01 \\
\hline 30 & 30 & Accept & 20 & 0.29 & 0.71 & 0.01 & 0.01 \\
\hline 30 & 30 & Accept & 15 & 0.30 & 0.7 & 0.01 & 0.01 \\
\hline 30 & 30 & Accept & 20 & 0.30 & 0.7 & 0.01 & 0.01 \\
\hline 30 & 30 & Accept & 20 & 0.31 & 0.69 & 0.01 & 0.01 \\
\hline 30 & 30 & Accept & 15 & 0.30 & 0.7 & 0.01 & 0.01 \\
\hline 30 & 30 & Accept & 15 & 0.29 & 0.71 & 0.01 & 0.01 \\
\hline 55 & 55 & Accept & 20 & 0.30 & 0.7 & 0.01 & 0.01 \\
\hline 55 & 55 & Accept & 20 & 0.29 & 0.71 & 0.01 & 0.01 \\
\hline 55 & 55 & Accept & 15 & 0.30 & 0.7 & 0.01 & 0.01 \\
\hline 55 & 55 & Accept & 10 & 0.31 & 0.69 & 0.01 & 0.01 \\
\hline 55 & 55 & Accept & 10 & 0.29 & 0.71 & 0.01 & 0.01 \\
\hline 55 & 55 & Accept & 20 & 0.31 & 0.69 & 0.01 & 0.01 \\
\hline 55 & 55 & Accept & 15 & 0.30 & 0.7 & 0.01 & 0.01 \\
\hline 55 & 55 & Accept & 15 & 0.29 & 0.71 & 0.01 & 0.01 \\
\hline 55 & 55 & Accept & 10 & 0.30 & 0.7 & 0.01 & 0.01 \\
\hline 58 & 58 & Accept & 20 & 0.31 & 0.69 & 0.01 & 0.01 \\
\hline 58 & 58 & Accept & 20 & 0.30 & 0.7 & 0.01 & 0.01 \\
\hline 78 & 78 & Accept & 20 & 0.31 & 0.69 & 0.01 & 0.01 \\
\hline 98 & 98 & Accept & 15 & 0.31 & 0.69 & 0.01 & 0.01 \\
\hline 98 & 98 & Accept & 20 & 0.31 & 0.69 & 0.01 & 0.01 \\
\hline 98 & 98 & Accept & 20 & 0.30 & 0.7 & 0.01 & 0.01 \\
\hline 98 & 98 & Accept & 20 & 0.29 & 0.71 & 0.01 & 0.01 \\
\hline 136 & 136 & Accept & 20 & 0.31 & 0.69 & 0.01 & 0.01 \\
\hline 136 & 136 & Accept & 20 & 0.30 & 0.7 & 0.01 & 0.01 \\
\hline 136 & 136 & Accept & 20 & 0.29 & 0.71 & 0.01 & 0.01 \\
\hline
\end{tabular}




\section{References}

[1] Ahmed, A. A., \& Traore, I. (2008). Performance Metrics and Model for Continuous Authentication Systems. submitted to the IEEE Transactions on Dependable and.

[2] O'Gorman, L. (2003). Comparing Passwords, Tokens, and Biometrics for User Authentication. IEEE, Dec. 03 Vol. 91, (pp. 2021-2040).

[3] Shepherd, S. (1995). Continuous authentication by analysis of keyboard typing characteristics. Security and Detection, European Convention, (pp. 111-114). Brighton.

[4] Ahmed, A., \& Traore, I. (2007). A New Biometric Technology Based on Mouse Dynamics. Dependable and Secure Computing, IEEE Transactions, (pp. 165-179).

[5] Loeb, V. (2001). Spy Case Prompts Computer Search. Washington Post, DC , A01-00.

[6] Maxion, R., \& Townsend, T. (2004). Masquerade detection augmented with error analysis. Reliability, IEEE Transactions (pp. 124-147). Pittsburgh: IEEE Reliability Society.

[7] Kim, H., \& Cha, S. (2005). Empirical evaluation of SVM-based masquerade detection using UNIX commands. Computer \& Security , 24, 160-168.

[8] Wang, K., \& S, S. (2003). One-class training for masquerade detection. 3rd IEEE Conference Data Mining Workshop on Data Mining for Computer Security.

[9] Schonlau, M., DuMouchel, W., Ju, W., Karr, A. F., Theus, M., \& Vardi, Y. (2001). Computer Intrusion: Detecting masquerades. Statistical Science , I (16), 58-74.

[10] Maxion, R., \& Townsend, T. (2002). Masquerade detection using truncated command lines. Dependable Systems and Networks. DSN 2002. Proceedings. International Conference, (pp. 219-228). Pittsburgh.

[11] Axelsson, S. (2000, March 14). Intrusion Detection Systems: A Survey and Taxonomy. 1-27. Sweden.

[12] Armstrong, D., \& Peile, C. (2005). Perimeter intruder detection systems performance standard. Security Technology, 2005. CCST '05. 39th Annual 2005 International Carnahan Conference, (pp. 33-36). Las Palmas.

[13] Cannady, J. (1998). Artificial Neural Networks for Misuse Detection. Retrieved from http://csrc.nist.gov/nissc/1998/proceedings/paperF13.pdf

[14] Ghosh, A. K., \& Schwartzbard, A. (1999). A study in using neural networks for anomaly and misuse detection. Proceedings of the 8th conference on USENIX Security Symposium - Volume 8, 12-12. 
[15] Jali, K. A., Kamarudin, M. H., \& Masrek, M. N. (2010). Comparison of Machine Learning algorithms performance in detecting network intrusion. Networking and Information Technology (ICNIT), International Conference, (pp. 221-226). Manila, Philippines.

[16] Kemmerer, R. A., \& Vigna, G. (2002). Intrusion detection: A brief history and overview. Computer 35 (SUPPL) , 27-30.

[17] Lin, M., Miikkulainen, R., \& Ryan, J. (1998, June). Intrusion Detection with Neural Networks. Advances in Neural Information Processing Systems .

[18] Liu, Z., Florez, G., \& Bridges, S. (2002). A comparison of input representations in neural networks: a case study in intrusion detection. Neural Networks. IJCNN '02. Proceedings of the 2002 International Joint Conference on, (pp. 1708-1713). Honolulu, HI.

[19] Zhang, P. (2005). Neural Networks. In Data Mining and Knowledge Discovery Handbook (pp. 487507). Springer US.

[20] Mitchell, T. (1997). Machine Learning. The McGraw-Hill Companies, Inc.

[21] Kim, S.-B., Han, K.-S., Rim, H.-C., \& Myaeng, S. H. (2006). Some Effective Techniques for Naive Bayes Text Classification. Knowledge and Data Engineering, IEEE Transactions, (pp. 1457-1466). Los Angeles.

[22] Michie, D., Spiegelhalter, D. J., \& Taylor, C. C. (1994). Machine learning, neural and statistical classification. New York: Ellis Horwood.

[23] Almeida, T., Yamakami, A., \& Almeida, J. (2009). Evaluation of Approaches for Dimensionality Reduction Applied with Naive Bayes Anti-Spam Filters. Machine Learning and Applications. ICMLA '09. International Conference, (pp. 517-522). Miami Beach, FL.

[24] Yang, Z., Nie, X., Xu, W., \& Guo, J. (2006). An Approach to Spam Detection by Naive Bayes Ensemble Based on Decision Induction. Intelligent Systems Design and Applications. ISDA '06. Sixth International Conference, (pp. 861-866). Jinan.

[25] Joachims, T. (1996). A probabilistic analysis of the Rocchio algorithm with TFIDF for text categorization. Carnegie Mellon University: Computer Science Technical Report CMU-CS-96-118.

[26] Maxion, R. (2003). Masquerade detection using enriched command lines. Dependable Systems and Networks. Proceedings. 2003 International Conference, (pp. 5-14).

[27] Maxion, R., \& Tan, K. (2000). Benchmarking anomaly-based detection systems. Dependable Systems and Networks, 2000. DSN 2000. Proceedings International Conference, (pp. 623-630). New York, NY.

[28] Jiang, L., Zhang, H., \& Cai, Z. (2009). A Novel Bayes Model: Hidden Naive Bayes. Knowledge and Data Engineering, IEEE Transactions, (pp. 1361-1371).

[29] Garg, A., Rahalkar, R., Upadhyaya, S., \& Kwiat, K. (2006). Profiling Users in GUI Based Systems for Masquerade Detection. West Point: Information Assurance Workshop IEEE. 
[30] Ghosh, A. K., Schwartzbard, A., \& Schatz, M. (1999). Learning Program Behavior Profiles for Intrusion Detection.

[31] Ghosh, A. K., Wanken, J., \& Charron, F. (1998). Detecting anomalous and unknown intrusions against programs. In Proceedings of the 1998 Annual Computer Security Applications Conference .

[32] Heywood, M., Lichodzijewski, P., \& Zincir-Heywood, N. (2001). Host-Based Intrusion Detection Using Self-Organizing Maps. Retrieved from http://users.cs.dal.ca/ mheywood/Xfiles/Publications/PeterSOM-ids.pdf

[33] Hoglund, A. J., Hatonen, K., \& Sorvari, A. S. (2000). A computer host-based user anomaly detection system using theself-organizing map. Proceedings of the IEEE-INNS-ENNS International Joint Conference on , 411 - 416.

[34] Wang, W., Guan, X., \& Zhan, X. (2004). Profiling program and user behaviors for anomaly intrusion detection based on non-negative matrix factorization. Decision and Control, 2004. CDC. 43rd IEEE Conference, (pp. 99 - 104 Vol.1). Atlantis.

[35] Marin, J., Ragsdale, D., \& Sirdu, J. (2001). A hybrid approach to the profile creation and intrusion detection. DARPA Information Survivability Conference \& Exposition II. DISCEX '01. Proceedings, (pp. 6976). Anaheim.

[36] Greenberg, S. (1988). Using Unix: collected traces of 168 users. Calgary: Research report 88/333/45, Department of Computer Science, University of Calgary.

[37] Jian, Z., Shirai, H., Takahashi, I., Kuroiwa, J., Odaka, T., \& Ogura, H. (2007). A Hybrid Command Sequence Model for Anomaly Detection. Advances In Knowledge Discovery and Data Mining , 4426 (1), 108-118.

[38] Seo, J., \& Cha, S. (2007). Masquerade detection based on SVM and sequence-based user commands profile. 2nd ACM symposium on Information, computer and communications security, (pp. 398-400). Singapore.

[39] Zhang, H., \& Sheng, S. (2004). Learning weighted naive Bayes with accurate ranking. Data Mining. ICDM '04. Fourth IEEE International Conference, (pp. 567-570).

[40] Masrom, M., \& Ismail, Z. (2008). Computer security and computer ethics awareness: A component of management information system. Information Technology. ITSim 2008. International Symposium, (pp. 1-7). Kuala Lumpur.

[41] Yurcik, W., \& Liu, C. (2005). A first step toward detecting SSH identity theft in HPC cluster environments: discriminating masqueraders based on command behavior. Cluster Computing and the Grid, 2005. CCGrid 2005. IEEE International Symposium, (pp. 111 - 120). Cardiff, UK.

[42] Bishop, M. (2009). Reflections on UNIX Vulnerabilities. Computer Security Applications Conference. ACSAC '09. Annual, (pp. 161-184). Honolulu, HI. 
[43] Robbins, A. (2005). Unix in a Nutshell (4th ed.). (M. Loukides, Ed.) Sebastopol, United States of America: O'Reilly Media Inc.

[44] Lang, K. (1995). Newsweeder: Learning to filter netnews. 12th International Conference on Machine Learning (pp. 331-339). San Francisco: Morgan Kaufmann Publishers.

[45] Leng, C., Wang, S., \& Wang, H. (2009). Learning Naive Bayes Classifiers with Incomplete Data. Artificial Intelligence and Computational Intelligence. AICI '09. International Conference, (pp. 350-353). Shanghai.

[46] Brosso, I., Ferreira, F., Bressan, G., \& Ruggiero, W. (2010). Known User Continuous Authentication System. Consumer Communications and Networking Conference (CCNC), 2010 7th IEEE, (pp. 1-2). Las Vegas, NV.

[47] Ferro, M., Pioggia, G., Tognetti, A., Dalle Mura, G., \& De Rossi, D. (2009). Event Related Biometrics: Towards an Unobtrusive Sensing Seat System for Continuous Human Authentication. Intelligent Systems Design and Applications. ISDA '09. Ninth International Conference on, (pp. 679-682). Pisa.

[48] Liu, J., Yu, F., Lung, C.-H., \& Tang, H. (2008). A Framework of Combining Intrusion Detection and Continuous Authentication in Mobile Ad Hoc Networks. Communications. ICC '08. IEEE International Conference on , (pp. 1515-1519). Beijing.

[49] Kohonen, T. (2001). Self-Organizing Maps (3rd ed.). New York, United States of America: Springer. [50] Lane, T., \& Brodley, C. E. (2003). An Empirical Study of Two Approaches to Sequence Learning for Anomaly Detection. Machine Learning Vol. 51, 73-107.

[51] Johnson, R. A. (2005). Miller \& Freund's Probability and Statistics for Engineers. Toronto: Pearson Education, Inc.

[52] Wan, M. D., Wu, H.-C., Kuo, Y.-W., Marshall, J., \& Huang, S.-H. (2008). Detecting Masqueraders Using High Frequency Commands as Signatures. Advanced Information Networking and Applications Workshops. AINAW 2008. 22nd International Conference, (pp. 596-601). Okinawa. 